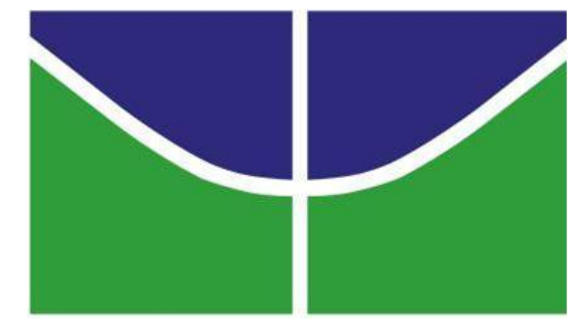

Universidade de Brasília Instituto de Ciências Exatas

Departamento de Matemática

\title{
Representações fechadas por estado de grupos metabelianos tipo entrelaçado
}

por

Alex Carrazedo Dantas

Orientador: Professor Doutor Said Najati Sidki

Brasília

2016 
Universidade de Brasília

Instituto de Ciências Exatas

Departamento de Matemática

\title{
Representações fechadas por estado de grupos metabelianos tipo entrelaçado
}

\author{
por \\ Alex Carrazedo Dantas \\ Orientador: Professor Doutor Said Najati Sidki
}

Brasília

2016 
Ficha catalográfica elaborada automaticamente, com os dados fornecidos pelo(a) autor(a)

Carrazedo Dantas, Alex

Representações fechadas por estado de grupos

$r$ metabelianos tipo entrelaçado / Alex Carrazedo Dantas; orientador Said Najati Sidki. -- Brasillia, 2016. $64 \mathrm{p}$.

Tese (Doutorado - Doutorado em Matemática) -Universidade de Brasília, 2016.

1. Automorfismos de árvores. 2. Representações fechadas por estado. 3. Grupos metabelianos fechados por estado. 4. Grupos tipo Lamplighter. I. Najati Sidki, Said, orient. II. Título. 


\title{
Representações fechadas por estado de grupos metabelianos tipo entrelaçado
}

\author{
por \\ Alex Carrazedo Dantas
}

Tese apresentada ao Corpo Docente do Programa de Pós-Graduação em Matemática-UnB, como requisito parcial para obtenção do grau de

\section{DOUTOR EM MATEMÁTICA}

Brasília, 27 de janeiro de 2016.

Comissão Examinadora:

\section{sind Sidki}

Prof. Dr. Said Najati Sidki - Orientador (MAT-UnB)

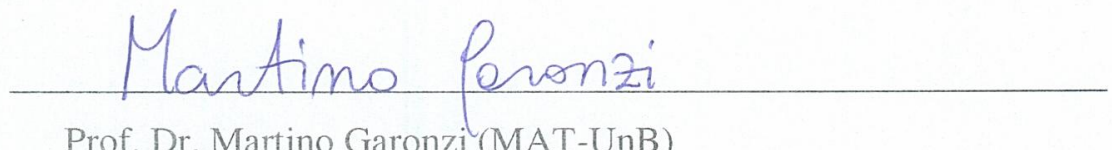

Prof. Dr. Martino Garonzi (MAT-UnB)

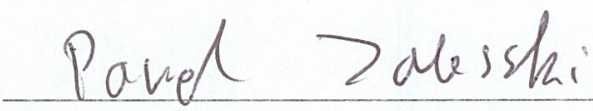

Prof. Dr. Pavel Zalesski (UnB)

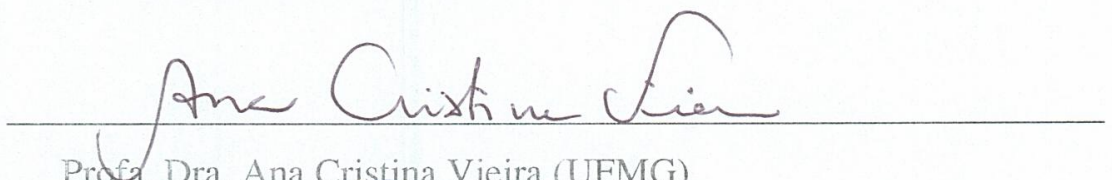

Prded. Dra. Ana Cristina Vieira (UFMG)

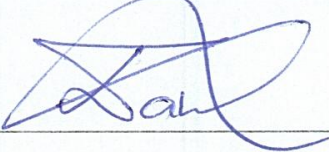

Profa. Dra. Dessislava Hristova Kochloukova (UNICAMP)

* O autor foi bolsista CNPq durante a elaboração desta tese. 
À minha mãe, Irene Carrazedo Dantas.

À minha família.

À minha namorada, Camila de Oliveira Vieira. 


\section{Agradecimentos}

À minha mãe, que apesar de tudo se mantém firme e é a grande conexão entre todos de nossa família. Ao meu pai. Aos meus irmãos Sandro, Sérgio, Márcio e Marcos e à minha irmã Márcia. À toda minha família.

À minha namorada Camila de Oliveira Vieira, que durante esses quatro anos se manteve ao meu lado, me apoiando. Aos seus pais Dona Mara e Seu Lino e à toda sua família.

Aos meus amigos do Mestrado Jorge e Thiago. Aos meus amigos de doutorado de maior convivência: Hiuri, Daiane, Juliana e Lais e aos amigos Bruno, Benedito, Agenor, Alex, Emerson e Raimundo.

Ao meu orientador Said Najati Sidki, que com toda paciência e sabedoria me auxiliou nesse trabalho e me ensinou muito nesses quatro anos de doutorado.

A todos os professores e funcionários do Departamento de Matemática da $\mathrm{UnB}$.

Aos membros da banca examinadora, pela disponibilidade de participar e pelas contribuições acerca da tese.

À CAPES e à CNPq pelo apoio financeiro. 
Neste trabalho estudamos representações fechadas por estado de grupos metabelianos tipo entrelaçado, com ênfase nos grupos tipo Lamplighter $G_{p, d}=C_{p} \prec C^{d}$. Tal estudo é motivado por uma representação fechada por estado de grau 2 do grupo Lamplighter $C_{2}$ \C, a qual foi utilizada para determinar seu espectro como um grupo de operadores lineares e, assim, dar um contra-exemplo de uma conjectura de Atiyah. No caso $d=1$, damos uma caracterização para as representações fechadas por estado de grau $p$ de $G_{p, 1}$. Para o caso $d>1$, demostramos que $G_{p, d}$ possui uma representação fechada por estado de grau $p^{2}$, mas não possui de grau $q, \operatorname{com} q$ primo. Além disso, demostramos que a representação de $C_{2}$ 乙 $C^{2}$ nesta família de representações é finita por estado.

Palavras-chave: Automorfismos de árvores, representação fechada por estado, grupos metabelianos tipo entrelaçado e grupos tipo Lamplighter. 
In this work we study state-closed representation of metabelian groups of wreath type, with emphasis on the Lamplighter groups of the type $G_{p, d}=C_{p} \prec C^{d}$. This study was motivated by a particular state-closed representation of degree 2 of the Lamplighter group $C_{2}$ \& $C$, which was used to determine the spectrum of $C_{2}$ \& as a group of linear operators and thus give a counterexample to a conjecture of Atiyah. In the case $d=1$, we characterize the state-closed representations of degree $p$ of the group $G_{p, 1}$. For the case $d>1$, we show the group $G_{p, d}$ has a stateclosed representation of degree $p^{2}$, but does not have a state-closed representation of degree $q$, where $q$ is prime number. Furthermore, we prove the representation obtained for $G_{2,2}$ is finite-state.

Keywords: Tree automorphisms, state-closed representation, Groups of Lamplighter type. 


\section{Índice de Notações}

$\begin{array}{ll}x^{y} & y^{-1} x y ; \\ X^{Y} & \left\langle x^{y} \mid x \in X, y \in Y\right\rangle ; \\ {[x, y]} & x^{-1} y^{-1} x y ; \\ |S| & \text { cardinalidade do conjunto } \mathrm{S} ; \\ H \leqslant G & H \text { é subgrupo de } G ; \\ H \unlhd G \text { ou } H \triangleleft G & H \text { é subgrupo normal de } G ; \\ G \simeq K & G \text { é isomorfo a } K ; \\ {[G: H]} & \text { índice do subgrupo } H \text { no grupo } G ; \\ \langle X\rangle & \text { subgrupo gerado por } X ; \\ {[A, B]} & \text { subgrupo }\langle[a, b]| a \in A \text { e } b \in B\rangle ; \\ G^{\prime} & {[G, G] ;} \\ G / N & \text { grupo quociente de } G \text { por }(\text { um subgrupo normal) } N ; \\ G_{1} \times \ldots \times G_{k} & \text { produto direto dos grupos } G_{1}, \ldots, G ; \\ G_{1} \oplus \ldots \oplus G_{k} & \text { soma direta dos grupos abelianos } G_{1}, \ldots, G_{k} ; \\ C r_{i \in I} G_{i} & \text { produto cartesiano dos grupos } G_{i}, i \in I ; \\ D r_{i \in I} G_{i} & \text { produto direto dos grupos } G_{i}, i \in I ; \\ N \rtimes H & \text { produto semidireto de } N \text { por } H ; \\ S_{m} & \text { grupo das permutações do conjunto }\{0,1, \ldots, m-1\} ; \\ S_{X} & \text { grupo das permutações do conjunto } X ; \\ D_{n} & \text { diedral de ordem } 2 n ; \\ D_{\infty} & \text { diedral infinito; } \\ X^{*} & \text { conjunto de todas as palavras finitas sobre o conjunto } X ; \\ \mathcal{T}_{m} & \text { árvore } m \text {-regular uni-raiz; } \\ & \end{array}$


$\mathcal{A}_{m} \quad$ grupo de automorfismo da árvore $\mathcal{T}_{m}$

$\mathcal{G}(A) \quad$ grupo de automorfismo da árvore $\mathcal{T}_{m}$ gerado pelos estados do autômato $A$;

$\operatorname{det}(M)$ determinante da matriz quadrada $M$;

$C \quad$ grupo cíclico infinito;

$C_{n} \quad$ grupo cíclico de ordem $n$. 
1 Automorfismos da árvore unirraiz $m$-regular $\quad 7$

1.1 A árvore uni-raiz $m$-regular e seus automorfismos . . . . . . . . . 7

1.1.1 A árvore uni-raiz $m$-regular $\mathcal{T}_{m} \ldots \ldots . \ldots 7$

1.1.2 O grupo $\mathcal{A}_{m}$ de automorfismos da árvore $\mathcal{T}_{m} \ldots \ldots . .8$

1.1.3 Autômatos .................. . 11

1.1.4 Subgrupos de $\mathcal{A}_{m} \ldots \ldots \ldots \ldots$. . . . . . . . . 15

1.2 Representações como grupo de automorfismos da árvore . . . . . 18

1.2.1 Representações de grau $m$. . . . . . . . . . . . . . 18

1.2.2 Representaçõoes fechadas por estado . . . . . . . . . . 19

2 Endomorfismos de grupos metabelianos tipo entrelaçado 24

2.1 Endomorfismos de produtos semidiretos . . . . . . . . . . . 24

2.2 Endomorfismos de grupos tipo Lamplighter . . . . . . . . . . . 27

2.3 Não existência de endomorfismo simples de grau primo para $G_{p, d}$,

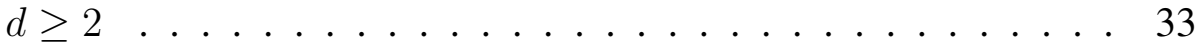

3 Representações fechadas por estado de grupos Lamplighter generalizados

3.1 Representações de grupos Lamplighter generalizados . . . . . . . 36

3.2 Representações do grupo Lamplighter $C_{p} \succ C \ldots 39$

3.3 Não existência de representação de grau primo para um grupo abeliano livre de rank infinito . . . . . . . . . . . . . . . 42 
3.4 Não existência de representação de grau primo para $C$ \ $C \ldots 45$

4 Representações de $G_{p, d}(d>1)$ de grau $p^{2} \quad 47$

4.1 Endomorfismo de grau $p^{2}$ em $G_{p, d} \ldots \ldots$. . . . . . . . . . 47

4.2 Representação fechada por estado de grau $p^{2}$ de $G_{p, d}$. . . . . . . 57

4.3 Representação fechada por estado de grau 4 de $G_{2,2}$. . . . . . . 59

Referências 


\section{Introdução}

Dizemos que um grupo $G$ possui uma representação como grupo de automorfismos da árvore uni-raiz $m$-regular $\mathcal{T}_{m}$ se existe um homomorfismo $\varphi$ de $G$ no grupo de automorfismo $\mathcal{A}_{m}$ de $\mathcal{T}_{m}$. O número $m$ é dito ser o grau da representação. Dizemos que $\varphi$ é fiel se $\varphi$ é um monomorfismo. Tal representação é também chamada de transitiva, fechada por estado ou finita por estado se $G^{\varphi}$ é, respectivamente, transitivo, fechado por estado ou finito por estado. Quando não houver confusão, chamaremos tanto $\varphi$ quanto $G^{\varphi}$ de representação de grau $m$ de $G$. Se existir um homomorfismo $f: H \rightarrow G$, chamado de endomorfismo virtual, onde $H$ é um subgrupo de índice $m$ em $G$, então uma construção recursiva usando $f$ produz uma representação transitiva e fechada por estado de grau $m$ de $G$. O núcleo dessa representação sobre árvore é o subgrupo de $G$ gerado por todos subgrupos de $H$, normais em $G$ e $f$-invariantes, ou seja, o subgrupo de $G$ dado por $\left\langle K \mid K \leqslant H, K \triangleleft G, K^{f} \leqslant K\right\rangle$. Se tal núcleo é trivial, $f$ é chamado de endomorfismo simples. Neste caso, dizemos que $G$ possui uma representação transitiva fechada por estado de grau $m$ ou, quando não houver confusão, simplesmente representação fechada por estado.

Nestas notas, estudamos representações transitivas e fechadas por estado de grupos metabelianos tipo entrelaçados, isto é, grupos que podem ser escritos como um produto entrelaçado restrito $B \nmid X$, onde $B$ e $X$ são grupos abelianos. Especificamente, estudamos representações de grupos tipo Lamplighter $G_{p, d}=C_{p} \imath C^{d}$, onde $C_{p}$ é o grupo cíclico com $p$ elementos, $p$ um número primo, $C$ é o grupo cíclico infinito, $d$ um inteiro positivo e $C^{d}$ a soma direta de $d$ cópias de $C$. O grupo $G_{2,1}=C_{2}$ 乙 $C$ é reconhecido com o nome pitoresco de grupo Lamplighter. Os 
grupos $G_{2, d}$ aparecem em [15, página 480] como exemplos não triviais possuindo propriedades probabilísticas em caminhos randômicos sobre grupos discretos.

Uma importante aplicação do grupo Lamplighter apareceu no artigo On a conjecture of Atiyah em 2000 de R. Grigorchuck, P. Linnel, Th. Schick e A. Zuk, [10]. Nele os autores dão uma resposta negativa para uma conjectura de M. F. Atiyah proposta em 1976 em seu artigo Elliptic operators, discrete groups and von Neumamm Algebras, [1]. Nesse artigo, Atiyah introduz os $L^{2}$-números de Betti $b_{(2)}^{i}(M)$ de uma variedade riemanniana compacta $M$, com $i$ um inteiro não negativo. Tomando fin $^{-1}(G)$ como o subgrupo do grupo aditivo dos números racionais $\mathbb{Q}$ gerado por todos os inversos das ordens de todos elementos de ordens finitas do grupo $G$, ele conjectura que

Conjectura. ([1]) Sejam $M$ uma variedade compacta e $\pi(M)$ seu grupo fundamental. Então $b_{(2)}^{i}(M) \in \operatorname{fin}^{-1}(\pi(M))$, para todo inteiro $i$.

Vários textos apresentam resultados sobre esta conjectura e confirmam diferentes formas dela, confira [13] e [25] para mais detalhes. Entretanto, como dito no último parágrafo, tal conjectura não vale de maneira geral. Em [10] e [11], os autores demonstram que o grupo

$$
G=\left\langle a, x, y \mid a^{2}=[x, y]=\left[a^{x}, a\right]=a^{y}[a, x]=1\right\rangle
$$

é o grupo fundamental de uma variedade riemanniana compacta de dimensão 7 e $b_{(2)}^{3}(M)=\frac{1}{3} \notin \operatorname{fin}^{-1}(G)$. De fato $\frac{1}{3} \notin \mathrm{fin}^{-1}(G)$, pois todo elemento de ordem finita de $G$ tem ordem dois. Observando que o grupo $G$ é uma $H N N$-extensão ascendente do grupo Lamplighter $G_{2,1}$, para calcular $b_{(2)}^{3}(M)=\frac{1}{3}$, eles lançaram mão da representação transitiva e fechada por estado $\left\langle\alpha=(\alpha, \alpha \sigma), \sigma=\left(\begin{array}{ll}0 & 1\end{array}\right)\right\rangle$ de $G_{2,1}$ e, assim, de sua ação como um grupo de operadores lineares do espaço de Hilbert $\partial \mathcal{T}_{2}$, onde a fronteira $\partial \mathcal{T}_{m}$ de $\mathcal{T}_{m}$ é o conjunto de todas as sequências infinitas sobre $\{0,1, \ldots, m-1\}$. Veja [1], [10], [11], [12], [18] e [25] para mais informações.

Desde então vários outros artigos sobre generalizações do grupo Lamplighter apareceram, confira [3], [6], [14] e [24]. Em destaque, Silva e Steinberg, em [24], chamam os grupos da forma $B$ ? $C$, com $B$ um grupo abeliano finito, de grupos Lamplighters generalizados e demonstram que tais grupos possuem uma representação transitiva fechada por estado e finita por estado de grau $|B|$. Em seguida, Kambites, Silva e Stenberg usando esta representação computaram os espectros de tais grupos em [14].

Nesse sentido, nos questionamos sobre a existência de uma representação transitiva fechada por estado de $G_{p, d}$. Demonstramos que o grupo $G_{p, d}, \operatorname{com} d>1$, 
possui uma representação transitiva e fechada por estado de grau $p^{2}$, enquanto que não existe tal representação de grau primo $q$, qualquer que seja o primo $q$. Isso tem certo contraste com, por um lado, o fato de $G_{p, 1}$ possuir uma representação transitiva fechada por estado de grau $p$ e, por outro, com o fato de $G_{p, d}$ possuir uma representação fiel finita por estado de grau $p$ independente de $d$, não necessariamente fechada por estado ou transitiva. Esse último fato foi estabelecido utilizando uma técnica geral chamada tree-wreathing, introduzida em [23] por S. N. Sidki.

Mais ainda, demonstramos que no caso $p=2$ e $d=2$ a representação transitiva fechada por estado de grau 4 de $G_{2,2}$ é finita por estado, a saber, 12 estados. Também classificamos em dois tipos as representações transitivas e fechadas por estado do grupo Lamplighter $G_{p, 1}$ (aqui usamos a nomenclatura, amplamente usada, grupo Lamplighter para $G_{p, 1}$, qualquer que seja o primo $p$ ).

No Capítulo 1, estabelecemos todas as preliminares necessárias para um bom entendimento dos resultados mencionados acima. Nele definimos a árvore unirraiz $m$-regular $\mathcal{T}_{m}$, seu grupo de automorfismos $\mathcal{A}_{m}$, autômatos, grupos fechados por estado e representação fechada por estado. Finalizamos esse capítulo, com uma discussão sobre o grupo $G=\langle\alpha=(\alpha, \alpha)(01)\rangle$ gerado pelo autômato

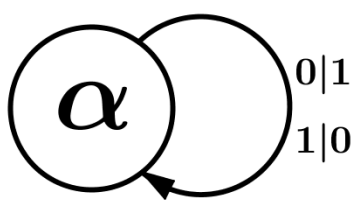

Figura 1

que não é induzido por um endomorfismo simples $f$, ou seja, não existem $H$ um subgrupo de índice 2 em $G, f$ um endomorfismo simples e $T$ um transversal de $H$ em $G$ que induzem essa representação transitiva e fechada por estado de $G$.

O Capítulo 2 se inicia com uma análise dos endomorfismos virtuais de grupos metabelianos tipo entrelaçado. Com as hipóteses de que $f: H \rightarrow G=B$ 乙 $X$ é um endomorfismo virtual simples sobre o grupo metabeliano tipo entrelaçado $G$, $A=\oplus_{X} B, A_{0}^{f} \leqslant A$, onde $A_{0}=H \cap A$ e $Y=A H \cap X$, então estabelecemos a proposição de que o par $(f, H)$ pode ser substituido pelo par $(\dot{f}, \dot{H})$, onde $\dot{H}=$ $A_{0} Y$ e $\dot{f}$ é um homomorfismo de $\dot{H}$ em $G$ e tais que $\dot{H}$ preserva normalidade e índice e $\dot{f}$ preserva simplicidade. Tal fato se mostrou de extrema importância em todo trabalho. Como exemplo, nesse mesmo capítulo utilizamos tal proposição para estabelecer os seguintes resultados de não existência: 
Teorema 0.0.1. Seja $G_{p, d}=C_{p} \succ C^{d}$, onde $C_{p}=\langle a\rangle$ é de ordem prima pe $C$ é o cíclico infinito. Sejam A ofecho normal de $\langle a\rangle, H$ um subgrupo de índice finito em $G$ e $f: H \rightarrow G$ um homomorfismo. Suponha que $H$ se projeta sobrejetivamente sobre $C^{d}$. Então f não é simples.

Teorema 0.0.2. Não existe endomorfismo simples $f: H \rightarrow G_{p, d}$ tal que o índice $\left[G_{p, d}: H\right]$ é primo.

Análogo a tal fato, em [3], é demonstrado que um grupo nilpotente finitamente gerado livre de torção de classe de nilpotência $c>1$ não admite uma representação transitiva fechada por estado de grau $p$. Por fim, o seguinte fato de existência é estabelecido.

Teorema 0.0.3. $O$ grupo $G=C_{n}^{k} \curlywedge C^{d}$, possui uma representação transitiva fechada por estado de grau $n^{k(n+d)}$, para todo $d \geq 2$.

Lembrando que um automorfismo $\alpha$ da árvore $\mathcal{T}_{m}$ pode ser escrito na forma $\alpha=\left(\alpha_{0}, \ldots, \alpha_{m-1}\right) \sigma, \alpha_{i} \in \mathcal{A}_{m}$ e $\sigma \in S_{m}$ é a permutação do primeiro nível da árvore. No Capítulo 3, provamos resultados gerais sobre as representações transitivas e fechadas por estado de um grupo Lamplighter $G_{p, 1}$.

Teorema 0.0.4. Suponha que $H$ é um subgrupo normal de $G_{p, 1}=\langle a\rangle \prec\langle x\rangle$ de índice $p$. Então cada representação fechada por estado de $G_{p, 1}$ sobre a árvore uniraiz p-regular com respeito a $H$ é reduzida a $\varphi: G_{p, 1} \rightarrow \mathcal{A}_{p}$, onde

$$
\begin{gathered}
a \mapsto a^{\varphi}=\sigma=(01 \ldots p-1) \\
x \mapsto x^{\varphi}=\xi=\left(\xi^{n}, \xi^{n} \sigma^{u(\xi)}, \ldots, \xi^{n} \sigma^{u(\xi)(p-1)}\right)
\end{gathered}
$$

para algum inteiro $n$ e algum polinômio de Laurent $u(x) \in \mathbb{K}\langle x\rangle$, com $\mathbb{K}$ um corpo com pelementos, tais que $m d c(p, n)=1$ e $u(1) \neq 0$.

Produzimos também representações concretas de $G=G_{p, 1}$ no caso do subgrupo $H$ não ser necessariamente um subgrupo normal de $G$.

Teorema 0.0.5. Suponha que $H$ é um subgrupo de $G_{p, 1}$ de índice $p$. Então cada representação fechada por estado de $G_{p, 1}$ sobre a árvore uniraiz p-regular com respeito a $H$ é reduzida a $\varphi: G_{p, 1} \rightarrow \mathcal{A}_{p}$, dada por

$$
\begin{gathered}
a \mapsto a^{\varphi}=\sigma=(01 \ldots p-1) \\
x \mapsto x^{\varphi}=\xi=\left(\xi^{n}, \xi^{n} \sigma^{u(\xi)}, \ldots, \xi^{n} \sigma^{u(\xi)(p-1)}\right) \tau
\end{gathered}
$$

onde $\tau: i \mapsto i c(\bmod p), \operatorname{com} c \in\{1, \ldots, p\}$ e ne o polinômio de Laurent $u(x) \in$ $\mathbb{K}\langle x\rangle$ são tais que $\operatorname{mdc}(p, n)=1$ e $u(c) \neq 0$. 
Analisando ainda a existência de representações de grupos da forma $B$ ? $C$, onde $B$ é um grupo abeliano e $C$ o cíclico infinito, demonstramos que:

Teorema 0.0.6. O grupo $C \imath C$ não possui uma representação fechada por estado de grau , com p primo.

Este resultado segue de:

Teorema 0.0.7. Não existe uma representação fechada por estado de grau primo p de um grupo abeliano livre de rank infinito.

o que responde parcialmente uma questão proposta por A. M. Brunner e S. N. Sidki em [3, página 457]. Nela os autores perguntam sobre a existência de uma representação transitiva fechada por estado de um grupo abeliano livre de rank infinito.

O Capítulo 4 trata de representação transitiva e fechada por estado de grau $p^{2}$ do grupo $G_{p, d}$, com $d \geq 2$.

Teorema 0.0.8. Seja $G_{p, d}=C_{p} \imath C^{d}=\langle a\rangle \imath\left\langle x_{1}, x_{2}, \ldots, x_{d}\right\rangle$, com $d \geq 1$. Considere $H=G^{\prime} Y$, onde $Y=\left\langle x_{1}^{p}, x_{2}, \ldots, x_{d}\right\rangle$. Então a função

$$
\begin{gathered}
a^{x_{1}-1} \mapsto a^{i}, a^{x_{1}^{2}-1} \mapsto a^{2}, \ldots, a^{x_{1}^{p-1}-1} \mapsto a^{p-1}, a^{z-1} \mapsto 1, \forall z \in Y, \\
x_{1}^{p} \mapsto x_{2}, x_{2} \mapsto x_{3}, \ldots, x_{d-1} \mapsto x_{d}, x_{d} \mapsto x_{1},
\end{gathered}
$$

estende-se a um endomorfismo $f: H \rightarrow G_{p, d}$ simples.

Finalmente, estabelecemos uma representação concreta da ação de $G_{2,2}$ sobre a árvore de grau 4.

Teorema 0.0.9. Sejam

$$
\begin{gathered}
\sigma=(01)(23), \\
\alpha=\left(e, \sigma^{\beta^{-1}}, \beta, \sigma^{\beta^{-1}} \beta\right)(02)(13), \\
\beta=\left(\alpha, \alpha, \alpha, \sigma^{\beta^{-1}+\beta^{-1} \alpha^{-1}} \alpha\right),
\end{gathered}
$$

automorfismo de $\mathcal{T}_{4}$. Então $G_{2,2}$ é isomorfo ao grupo transitivo fechado por estado $\langle\sigma, \alpha, \beta\rangle$.

Mais que isso, demonstramos que essa ação é finita por estado, ou seja, o grupo $G_{2,2}$ é gerado pelos estados do autômato 


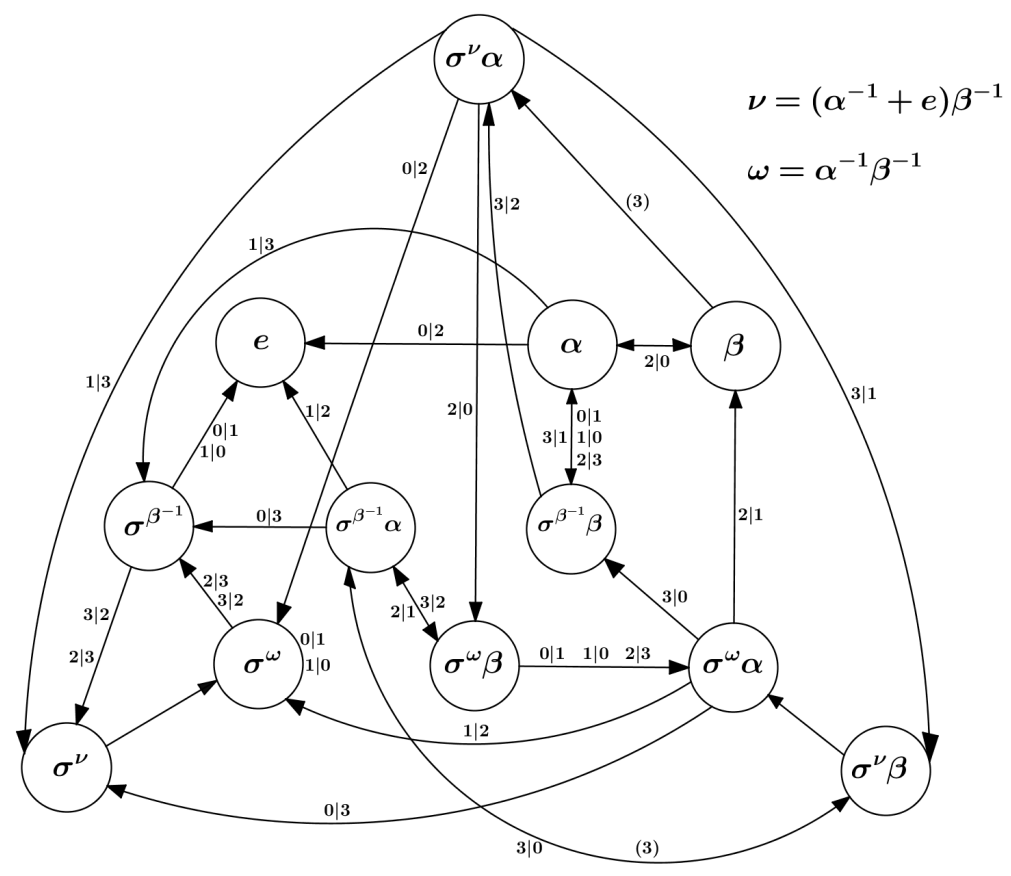

Figura 2: Autômato de $C_{2} \succ C^{2}$.

Todas as figuras deste trabalho foram feitas no software livre GeoGebra. 
CAPÍTULO 1

\section{Automorfismos da árvore unirraiz $m$-regular}

Neste capítulo definiremos a árvore unirraiz $m$-regular e estudaremos seus automorfismos.

\subsection{A árvore uni-raiz $m$-regular e seus automorfis- mos}

\subsubsection{A árvore uni-raiz $m$-regular $\mathcal{T}_{m}$}

Sejam $m$ um inteiro positivo e $Y$ o conjunto $\{0, \ldots, m-1\}$. Considere $\mathcal{M}=$ $\mathcal{M}(Y)$ o conjunto de todas as palavras de comprimento finito em $Y$. O conjunto $\mathcal{M}$ possui uma estrutura natural de semigrupo, onde a operação é a concatenação de palavras e o elemento neutro dessa operação é a palavra vazia $\emptyset$. Denote por $|u|$ o comprimento da palavra $u$ de $\mathcal{M}$.

Definição 1.1.1. A árvore unirraiz m-regular $\mathcal{T}_{m}=\mathcal{T}(Y)$ é definida pelo grafo $\left(V\left(\mathcal{T}_{m}\right), E\left(\mathcal{T}_{m}\right)\right)$, com $V\left(\mathcal{T}_{m}\right)=\mathcal{M}$ e um par ordenado $(u, v)$ está em $E\left(\mathcal{T}_{m}\right)$ se, e somente se, $v=$ uy, para algum y em $Y$, onde $u, v \in \mathcal{M}$.

Dado um inteiro não negativo $n$, chamaremos o conjunto de todas as palavras de comprimento $n$ de Nível $n$ da árvore $\mathcal{T}_{m}$. Assim, o Nível 0 de $\mathcal{T}_{m}$ é o conjunto formado apenas pela palavra vazia $\emptyset$, o Nível 1 é o conjunto $Y$, o Nível 2 o 
conjunto $\{00,01, \ldots, 0(m-1), \ldots,(m-1) 0,(m-1) 1, \ldots,(m-1)(m-1)\}$ e assim por diante. Assim o Nível $n$ da árvore $\mathcal{T}_{m}$ é o conjunto

$$
\{u \in \mathcal{M} ;|u|=n\}
$$

Quando $m=2$, chamaremos a árvore $\mathcal{T}_{2}$ de árvore binária, se $m=3$, de árvore ternária e assim por diante. Graficamente, a árvore binária pode ser vista como:

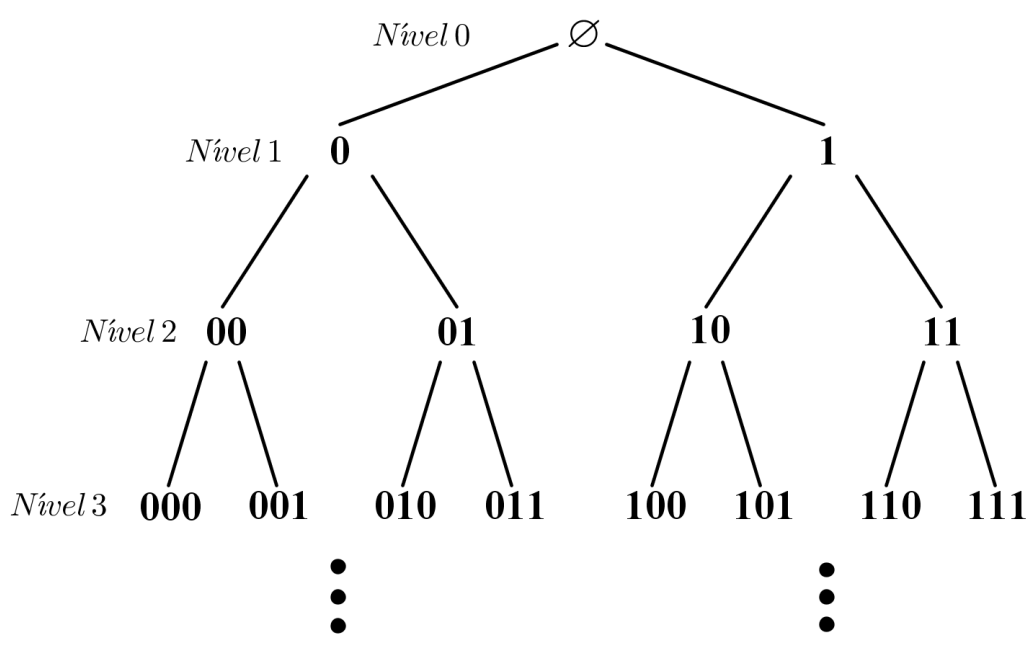

Figura 1.1: Árvore Binária $\mathcal{T}_{2}$

\subsubsection{O grupo $\mathcal{A}_{m}$ de automorfismos da árvore $\mathcal{T}_{m}$}

Antes de estudarmos o grupo de automorfismo de uma árvore unirraiz $m$-regular, vamos introduzir o conceito de produto entrelaçado (wreath product). Considere $\left\{G_{\lambda} \mid \lambda \in \Lambda\right\}$ uma família de grupos, onde $\Lambda$ é um conjunto de índices. O produto cartesiano dos $G_{\lambda}$ 's é definido por

$$
C r_{\lambda \in \Lambda} G_{\lambda}=\left\{\left(g_{\lambda}\right)_{\lambda \in \Lambda} \mid g_{\lambda} \in G_{\lambda}\right\}
$$

Munido com a operação

$$
\left(g_{\lambda}\right)_{\lambda \in \Lambda}\left(h_{\lambda}\right)_{\lambda \in \Lambda}=\left(g_{\lambda} h_{\lambda}\right)_{\lambda \in \Lambda}
$$


o conjunto $C r_{\lambda \in \Lambda} G_{\lambda}$ é um grupo, onde o elemento neutro é $\left(e_{\lambda}\right)_{\lambda \in \Lambda}$, sendo $e_{\lambda} \mathrm{o}$ elemento neutro de $G_{\lambda}$. O produto direto dos $G_{\lambda}$ 's é o subgrupo $D r_{\lambda \in \Lambda} G_{\lambda}$ de $C r_{\lambda \in \Lambda} G_{\lambda}$ dado por todos os elementos $\left(x_{\lambda}\right)_{\lambda \in \Lambda}$, onde $x_{\lambda} \neq e_{\lambda}$ para uma quantidade finita de índices $\lambda$. Claramente $D r_{\lambda \in \Lambda} G_{\lambda}$ é um subgrupo de $C r_{\lambda \in \Lambda} G_{\lambda}$. Note que se $\Lambda$ é finito, então $C r_{\lambda \in \Lambda} G_{\lambda}=D r_{\lambda \in \Lambda} G_{\lambda}$.

Considere $K$ um grupo, $\Lambda$ um conjunto e $H$ um grupo que age em $\Lambda$. Denote por $\varphi: H \rightarrow S_{\Lambda}$ a ação de $H$ em $\Lambda$, onde $S_{\Lambda}$ é o conjunto de todas as bijeções de $\Lambda$. O produto entrelaçado irrestrito de $K$ por $H$ com relação a é definido por

$$
K w r_{\varphi} H=\left(C r_{\lambda \in \Lambda} K\right) \rtimes_{\varphi} H
$$

onde

$$
\left(k_{\lambda}\right)_{\lambda \in \Lambda}^{h}=\left(k_{\lambda^{h \varphi}}\right)_{\lambda \in \Lambda},
$$

para todo $h \in H$ e todo $\lambda \in \Lambda$. O produto entrelaçado restrito de $K$ por $H$ com relação a $\varphi$ é definido por

$$
K \imath_{\varphi} H=\left(D r_{\lambda \in \Lambda} K\right) \rtimes_{\varphi} H
$$

onde

$$
\left(k_{\lambda}\right)_{\lambda \in \Lambda}^{h}=\left(k_{\lambda^{h \varphi}}\right)_{\lambda \in \Lambda},
$$

para todo $h \in H$ e todo $\lambda \in \Lambda$. Quando não houver confusão de qual é a ação de $H$ sobre $\Lambda$, supriremos $\varphi$ da notação. Para mais detalhes consulte [21].

Um automorfismo $\alpha$ de $\mathcal{T}_{m}$ é um morfismo de grafos bijetor $\alpha: \mathcal{T}_{m} \rightarrow \mathcal{T}_{m}$ que preserva comprimento de vértices. Com a operação de composição de funções, o conjunto de todos os automorfismos de $\mathcal{T}_{m}$ é um grupo, denotado por $\mathcal{A}_{m}$.

Exemplo 1.1.2. Dada uma permutação $\sigma$ de $Y$, podemos estendê-la a um automorfismo $\bar{\sigma}$ de $\mathcal{T}_{m}$, pondo:

$$
\begin{gathered}
(\emptyset) \bar{\sigma}=\emptyset \\
(y u) \bar{\sigma}=y^{\sigma} u
\end{gathered}
$$

para todo $y \in Y$ e para todo $u \in \mathcal{M}$. Para simplificar notação, vamos denotar a extensão $\bar{\sigma}$ de $\sigma$ simplismente por $\sigma$.

Por outro lado, dado um automorfismo $\alpha$ de $\mathcal{T}_{m}$, temos que $\alpha$ induz uma permutação $\sigma(\alpha)$ em $Y$. Basta considerar $\sigma(\alpha)$ igual a restrição $\alpha: Y \rightarrow Y$. Agora, podemos considerar a extensão $\sigma(\alpha)$, como no Exemplo 1.1.2. Logo a composição

$$
\alpha(\sigma(\alpha))^{-1}
$$


possui ação trivial no primeiro nível da árvore $\mathcal{T}_{m}$, ou seja, $(y) \alpha(\sigma(\alpha))^{-1}=y$, para todo $y \in Y$. Desta forma, podemos olhar para a composição $\alpha(\sigma(\alpha))^{-1}$ como

$$
\alpha(\sigma(\alpha))^{-1}=\left(\alpha_{0}, \ldots, \alpha_{m-1}\right),
$$

onde cada $\alpha_{y}$, com $y=0, \ldots, m-1$, é um morfismo bijetor da árvore

$$
y \mathcal{T}_{m}=\left(y V\left(\mathcal{T}_{m}\right), y E\left(\mathcal{T}_{m}\right)\right)
$$

onde $y V\left(\mathcal{T}_{m}\right)=\{y u ; u \in \mathcal{M}\}$ e $y E\left(\mathcal{T}_{m}\right)=\left\{(y u, y v) ;(u, v) \in E\left(\mathcal{T}_{m}\right)\right\}$. De $y \mathcal{T}_{m}$ ser isomorfo a $\mathcal{T}_{m}$, como grafos, podemos identificar $\alpha_{y}$ como um automorfismo de $\mathcal{T}_{m}$, daí

$$
\alpha=\left(\alpha_{0}, \ldots, \alpha_{m-1}\right) \sigma(\alpha),
$$

onde $\alpha_{y} \in \mathcal{A}_{m}$, para cada $y=0, \ldots, m-1$. Com essa identificação também podemos identificar o grupo $\mathcal{A}_{m}$ com o produto entrelaçado dele mesmo com o grupo $S_{m}$ das permutações de $Y$, ou seja,

$$
\mathcal{A}_{m}=\mathcal{A}_{m} \curlywedge S_{m}=\mathcal{A}_{m}^{m} \rtimes S_{m}=\left(\mathcal{A}_{m} \times \ldots \times \mathcal{A}_{m}\right) \rtimes S_{m},
$$

onde a ação de $S_{m}$ sobre $\mathcal{A}_{m} \times \ldots \times \mathcal{A}_{m}$ é sobre os índices. Assim dados $\sigma \in S_{m}$ e $\left(\alpha_{0}, \ldots, \alpha_{m-1}\right) \in \mathcal{A}_{m} \times \ldots \times \mathcal{A}_{m}$, segue que

$$
\sigma\left(\alpha_{0}, \ldots, \alpha_{m-1}\right)=\left(\alpha_{0^{\sigma}}, \ldots, \alpha_{(m-1)^{\sigma}}\right) \sigma .
$$

Portanto, se $\alpha=\left(\alpha_{0}, \ldots, \alpha_{m-1}\right) \sigma(\alpha)$ e $\beta=\left(\beta_{0}, \ldots, \beta_{m-1}\right) \sigma(\beta)$ são elementos de $\mathcal{A}_{m}$, segue que

$$
\alpha \beta=\left(\alpha_{0} \beta_{0^{\sigma(\alpha)}}, \ldots, \alpha_{m-1} \beta_{(m-1)^{\sigma(\alpha)}}\right) \sigma(\alpha) \sigma(\beta)
$$

e

$$
\alpha^{-1}=\left(\alpha_{0^{(\sigma(\alpha))^{-1}}}^{-1}, \ldots, \alpha_{(m-1)^{(\sigma(\alpha))^{-1}}}^{-1}\right)(\sigma(\alpha))^{-1} .
$$

Desses fatos, podemos enunciar a seguinte proposição.

Proposição 1.1.3. O grupo de automorfismos $\mathcal{A}_{m}$ de $\mathcal{T}_{m}$ é tal que

$$
\mathcal{A}_{m}=\mathcal{A}_{m}^{m} \rtimes S_{m}=\left(\mathcal{A}_{m} \times \ldots \times \mathcal{A}_{m}\right) \rtimes S_{m},
$$

onde cada elemento $\sigma$ de $S_{m}$ é visto pela sua extensão $\bar{\sigma}$, como no Exemplo 1.1.2. 
Por essa propriedade recursiva de $\mathcal{A}_{m}$, para se conhecer a ação de um automorfismo $\alpha=\left(\alpha_{0}, \ldots, \alpha_{m-1}\right) \sigma(\alpha)$ é necessário apenas conhecer a ação dos elementos de $\mathcal{A}_{m}$ no primeiro nível, fazendo

$$
(y u)^{\alpha}=y^{\sigma(\alpha)} u^{\alpha_{y}}
$$

onde $y=0,1, \ldots, m-1$.

Definição 1.1.4. Dado $\alpha=\left(\alpha_{0}, \ldots, \alpha_{m-1}\right) \sigma(\alpha)$ em $\mathcal{A}_{m}$, chamaremos ao conjunto definido recursivamente por

$$
Q(\alpha)=\left\{\alpha, \alpha_{0}, \ldots, \alpha_{m-1}\right\} \cup Q\left(\alpha_{0}\right) \cup \ldots \cup Q\left(\alpha_{m-1}\right)
$$

de conjunto de estados de $\alpha$. E a um elemento $\beta \in Q(\alpha)$ de um estado de $\alpha$.

Exemplo 1.1.5. Considere $\alpha=(e, \alpha) \sigma$ em $\mathcal{A}_{2}$, onde e é a identidade e $\sigma$ é a transposição $(01)$ em $S_{2}$. Seja $110 \in \mathcal{M}(\{0,1\})$. Assim

$$
(110)^{\alpha}=(110)^{(e, \alpha) \sigma}=1^{\sigma}(10)^{\alpha_{1}}=0(10)^{\alpha}=0(10)^{(e, \alpha) \sigma}=01^{\sigma} 0^{\alpha_{1}}=001 .
$$

Éfácil ver que $Q(\alpha)=\{e, \alpha\}$.

A nomenclatura "estado" de um automorfismo é herdada da ideia de autômato, que passaremos a definir na próxima subseção.

\subsubsection{Autômatos}

Os autômatos que consideraremos são invertíveis e os alfabetos de entrada e saída são um mesmo conjunto $\Gamma$ finito.

Formamente, um autômato $A$ é uma Máquina de Turing definida por uma quintupla $\left(Q, \Gamma, f, l, q_{0}\right)$, onde

- $\Gamma$ é um conjunto finito, chamado de alfabeto, em nosso caso, alfabeto de entrada e de saída;

- $Q$ é um conjunto de estados, onde cada $q \in Q$ é uma função bijetora $q: \Gamma \rightarrow \Gamma$;

- $f: Q \times \Gamma \rightarrow Q$ é uma função, chamada função de mudança de estados;

- $l: Q \times \Gamma \rightarrow \Gamma$ é uma função, chamada função de saída, definida por

$$
(q, a) l=(a) q
$$

para todo $a \in \Gamma$ e todo $q \in Q$; 
- $q_{0}$ é um estado de $Q$, chamado de estado inicial.

Também especificaremos um autômato por meio de um diagrama, de tal modo que existe uma aresta do estado $q$ ao estado $p$ e indexada por $a \mid b$ com uma seta apontada para $p$ se, e somente se, $(q, a) f=p$ e $(q, a) l=b$, onde $a, b \in \Gamma$. Graficamente temos

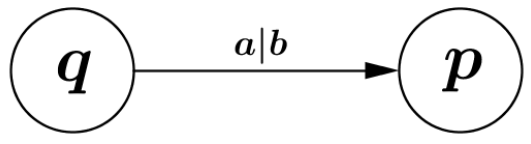

Figura 1.2: Diagrama 1.

O autômato inverso de $A$ é denotado por $A^{-1}$ e obtido ao comutar em cada aresta o índice $a \mid b$ por $b \mid a$, obtendo o diagrama.

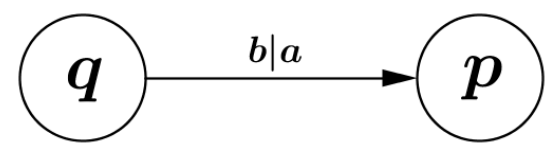

Figura 1.3: Diagrama 2.

Uma aresta pode ter dois ou mais índices ou até mesmo ter duas setas, como o diagrama abaixo.

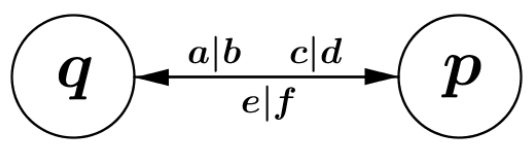

Figura 1.4: Diagrama 3.

Neste caso, temos que $(q, a) f=p,(q, c) f=p$ e $(q, a) l=b,(q, c) l=d$, mais ainda, $(p, e) f=q,(p, e) l=f$. O estado inicial é somente especificado para evitar confusão, assim colocaremos um autômato apenas por uma quadrupla $(Q, \Gamma, f, l)$.

Diremos que um autômato $A=(Q, \Gamma, f, l)$ é finito se $Q$ é finito. O exemplo abaixo é um exemplo de autômato finito.

Exemplo 1.1.6. Defina $Q=\{q, p\}, \Gamma=\{a, b\}$ e as funções $f: Q \times \Gamma \rightarrow Q e$ $l: Q \times \Gamma \rightarrow \Gamma$, respectivamente, por

$$
f:(q, a) \mapsto q,(q, b) \mapsto p,(p, a) \mapsto p,(p, b) \mapsto p
$$


$e$

$$
l:(q, a) \mapsto a,(q, b) \mapsto b,(p, a) \mapsto b,(p, b) \mapsto a .
$$

Defina o estado inicial $q_{0}=q$. Seja A o autômato dado pela quíntupla $(Q, \Gamma, f, l, q)$. Temos então uma representação em diagramas para A:

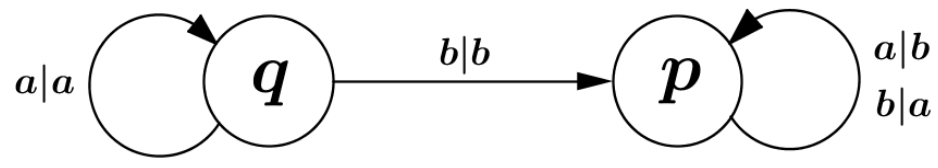

Figura 1.5: Diagrama do Autômato $A$.

Note que o autômato inverso $A^{-1}$ de A é o próprio autômato $A$.

Definição 1.1.7. Dois autômatos $\left(Q_{0}, \Gamma_{0}, f_{0}, l_{0}, q_{0}\right) e\left(Q_{1}, \Gamma_{1}, f_{1}, l_{1}, q_{1}\right)$ são equivalentes se existem bijeções $\Theta: Q_{0} \rightarrow Q_{1}$ e $\theta: \Gamma_{0} \rightarrow \Gamma_{1}$ tais que

$$
\left(q^{\Theta}, \gamma^{\theta}\right) f_{1}=\left((q, \gamma) f_{0}\right)^{\Theta}
$$

$e$

$$
\left(q^{\Theta}, \gamma^{\theta}\right) l_{1}=\left((q, \gamma) l_{0}\right)^{\theta},
$$

para quaisquer $\gamma \in \Gamma_{0} e q \in Q_{0}$.

Um automorfismo $\alpha=\left(\alpha_{0}, \ldots, \alpha_{m-1}\right) \sigma(\alpha)$ em $\mathcal{A}_{m}$ pode ser facilmente interpretado como um autômato. Basta tomar o autômato definido pela quíntupla $(Q, \Gamma, f, l, \alpha)$, onde $Q$ é o conjunto $Q(\alpha)$ dos estados de $\alpha$, $\Gamma$ é o conjunto $Y=$ $\{0,1, \ldots, m-1\}$, e as funções $f: Q(\alpha) \times Y \rightarrow Q(\alpha), l: Q(\alpha) \times Y \rightarrow Y$ são, respectivamente, definidas por

$$
f:(\beta, y) \mapsto \beta_{y}, l:(\beta, y) \mapsto y^{\sigma(\beta)},
$$

onde $y \in Y, \beta \in Q(\alpha)$ e $\sigma(\beta)$ é a permutação que $\beta$ induz em $Y$. $\operatorname{Logo}(\alpha, y) f=$ $\alpha_{y}$ e $(\alpha, y) l=y^{\sigma(\alpha)}, \operatorname{com} y=0,1, \ldots, m-1$. É daí que vem a nomenclatura "estados de $\alpha$ " para o conjunto $Q(\alpha)$.

Exemplo 1.1.8. Considere os automorfismos $\beta=(\beta, \beta) \sigma$ e $\alpha=(\alpha, \beta)$ em $\mathcal{A}_{2}$, onde $\sigma=(01)$. Temos que $\beta^{2}=\alpha^{2}=$ e e $Q(\alpha)=\{\alpha, \beta\}$. Podemos, então, definir o autômato $B$ pela quíntupla $(Q(\alpha),\{0,1\}, f, l, \alpha)$, onde $f$ e l são dados por

$$
f:(\alpha, 0) \mapsto \alpha_{0}=\alpha,(\alpha, 1) \mapsto \alpha_{1}=\beta,(\beta, 0) \mapsto \beta_{0}=\beta,(\beta, 1) \mapsto \beta_{1}=\beta
$$


$e$

$$
l:(\alpha, 0) \mapsto 0,(\alpha, 1) \mapsto 1,(\beta, 0) \mapsto 0^{\sigma}=1,(\beta, 1) \mapsto 1^{\sigma}=0 .
$$

O diagrama de $B$ é dado por

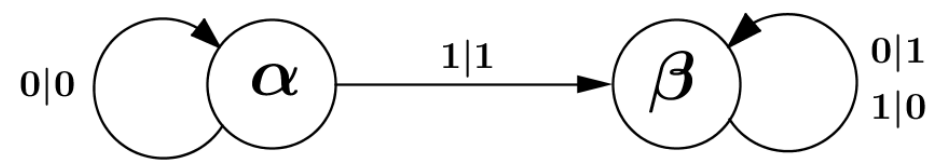

Figura 1.6: Diagrama do Autômato $B$.

O autômato B é equivalente ao autômato A do Exemplo 1.1.6. De fato, basta definir

$$
\Theta: q \mapsto \alpha, p \mapsto \beta, \quad \theta: a \mapsto 0, b \mapsto 1,
$$

que o resultado segue.

Por outro lado, cada estado $q$ de um autômato $A=(Q, \Gamma, f, l)$ pode ser visto como um elemento de $\mathcal{A}_{m}, \operatorname{com} m=|\Gamma|$. Basta corresponder o estado $q$ com o automorfismo

$$
\alpha_{q}=\left(\alpha_{0}, \ldots, \alpha_{m-1}\right) \sigma(q),
$$

onde $\Gamma=\left\{a_{0}, \ldots, a_{m-1}\right\}, \alpha_{i}$ é o automorfismo que corresponde ao estado $q_{i}$, com $q_{i}=\left(q, a_{i}\right) f$, e $\sigma(q)$ é a permutação de $\{0,1, \ldots, m-1\}$ definida por $i^{\sigma(q)}=j$, onde $j$ é tal que $\left(q, a_{i}\right) l=\left(a_{i}\right) q=a_{j}$.

Assim, dado um autômato $A=(Q, \Gamma, f, l)$, podemos olhar $Q$ como um subconjunto de $\mathcal{A}_{m}$, com $m=\Gamma$, e definir o subgrupo $\mathcal{G}(A) \leqslant \mathcal{A}_{m}$ gerado pelo autômato $A$ como

$$
\mathcal{G}(A)=\left\langle\alpha_{q} \mid q \in Q\right\rangle \leqslant \mathcal{A}_{m} .
$$

Em muitos casos, é interessante saber qual é a estrutura do grupo $\mathcal{G}(A)$, dado o autômato $A$. Como exemplo, A. Zuk classifica todos os grupos $\mathcal{G}(A)$, onde $A$ é um autômato com dois estados sobre um alfabeto binário. Ele demonstra que $\mathcal{G}(A)$ é isomorfo a um dos grupos $\{1\}, C_{2}, C_{2} \oplus C_{2}, C, D_{\infty}$ e $C_{2} \prec C$, onde $C_{2}$ é o cíclico de ordem 2, $C$ o cíclico infinito e $D_{\infty}$ é o grupo diedral infinito (veja [25, página 6] e [18, página 23]).

Exemplo 1.1.9. $O$ grupo $\mathcal{G}(A)$ do autômato A dado pelo diagrama 


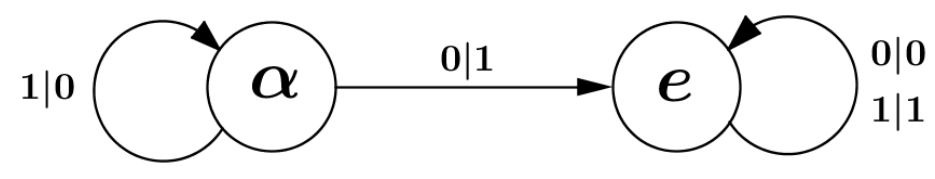

Figura 1.7

é isomorfo ao grupo cíclico infinito. De fato, note que

$$
\mathcal{G}(A)=\langle\alpha=(e, \alpha) \sigma, e=(e, e)\rangle,
$$

onde $\sigma=(01)$. Logo, basta mostrar que a tem ordem infinita, pois e é o automorfismo trivial. Isso segue do fato que $\alpha^{2 n}=\left(\alpha^{n}, \alpha^{n}\right)$ e $\alpha^{2 n+1}=\left(\alpha^{n}, \alpha^{n+1}\right) \sigma$, para todo número inteiro positivo. O automorfismo $\alpha=(e, \alpha) \sigma$ é chamado de máquina de adição binária.

Consulte [8] para mais detalhes sobre autômatos.

\subsubsection{Subgrupos de $\mathcal{A}_{m}$}

Dados um subgrupo $G$ de $\mathcal{A}_{m}, u \in \mathcal{M}(Y)$, com $Y=\{0,1, \ldots, m-1\}$, e $n$ um inteiro não negativo, definimos:

$$
\begin{gathered}
\operatorname{Fix}_{G}(u)=\left\{\alpha \in G \mid u^{\alpha}=u\right\}, \\
\operatorname{Stab}_{G}(n)=\left\{\alpha \in G\left|u^{\alpha}=u, \forall u \in \mathcal{M}(Y),\right| u \mid=n\right\}
\end{gathered}
$$

$\mathrm{e}$

$$
P(G)=\left\{\sigma(\alpha) \in S_{m} \mid \alpha \in G\right\} .
$$

Diremos que $G$ é transitivo, se $P(G)$ é um subgrupo transitivo de $S_{m}$.

\section{Fecho topológico}

Dado $\alpha \in \operatorname{Stab}_{\mathcal{A}_{m}}(1)$, segue que $\sigma(\alpha)=e$, ou seja,

$$
\alpha=\left(\alpha_{0}, \ldots, \alpha_{m-1}\right), \alpha_{i} \in \mathcal{A}_{m} .
$$

Assim, dado $\beta=\left(\beta_{0}, \ldots, \beta_{m-1}\right) \sigma(\beta) \in \mathcal{A}_{m}$, temos

$$
\alpha^{\beta}=\left(\alpha_{0}^{\beta_{0}}, \ldots, \alpha_{m-1}^{\beta_{m-1}}\right)^{\sigma(\beta)} \in \operatorname{Stab}_{\mathcal{A}_{m}}(1),
$$

onde estamos usando $\left(\gamma_{0}, \ldots, \gamma_{m-1}\right)^{\sigma}=\left(\gamma_{0^{\sigma}}, \ldots, \gamma_{(m-1)^{\sigma}}\right)$. Isso implica que o subgrupo $\operatorname{Stab}_{G}(1)=G \cap \operatorname{Stab}_{\mathcal{A}_{m}}(1)$ é normal em $G$, para todo subgrupo $G$ de 
$\mathcal{A}_{m}$. De maneira geral, $\operatorname{Stab}_{G}(n)$ é um subgrupo normal de $G$, para todo subgrupo $G$ de $\mathcal{A}_{m}$. Note também que o subgrupo $\operatorname{Stab}_{\mathcal{A}_{m}}(n)$ é um subgrupo com índice finito em $\mathcal{A}_{m}$. Mais que isso, $\mathcal{A}_{m}$ é o limite inverso de $\left\{\mathcal{A}_{m} / S t a b_{\mathcal{A}_{m}}(n)\right\}_{n=0}^{\infty}$, ou seja,

$$
\mathcal{A}_{m} \simeq \underset{\operatorname{Lim}}{\operatorname{Stab}_{\mathcal{A}_{m}}(n)}
$$

e assim cada automorfismo $\alpha \in \mathcal{A}_{m}$ pode ser visto como um produto infinito $\alpha_{0} \alpha_{1} \ldots \alpha_{n} \ldots$, onde $\alpha_{n} \in S t a b_{\mathcal{A}_{m}}(n)$. Logo o fecho topológico de $G$, denotado por $\bar{G}$, é o conjunto de todos os produtos infinitos $\alpha_{0} \alpha_{1} \ldots \alpha_{n} \ldots$, com $\alpha_{n} \in \operatorname{Stab}_{G}(n)$ (veja [20] para uma definição de limite inverso).

É fácil então ver que o fecho topológico de um subgrupo abeliano de $\mathcal{A}_{m}$ é também abeliano. Na verdade, o fecho topológico conserva a propriedade de ser verbal. Tal afirmação S. N. Sidki provou em [22, página 8].

\section{Fecho diagonal}

Outro fecho importante é o chamado fecho diagonal. Dado $\alpha \in \mathcal{A}_{m}$, definimos, recursivamente,

$$
\alpha^{(0)}=\alpha, \alpha^{(1)} \text { a } m \text {-upla }(\alpha, \ldots, \alpha) \text { e } \alpha^{(i+1)}=\left(\alpha^{(i)}\right)^{(1)} \text { para } i \geq 0 .
$$

Chamaremos de fecho diagonal de $G$ ao subgrupo $\widetilde{G}=\left\langle G^{(i)} \mid i \geq 0\right\rangle$. Denote $\alpha^{(i)}$ por $\alpha^{t^{i}}$, assim

$$
\alpha^{a_{0}}\left(\alpha^{a_{1}}\right)^{(1)}\left(\alpha^{a_{2}}\right)^{(2)}\left(\alpha^{a_{3}}\right)^{(3)} \ldots=\alpha^{a_{0}} \alpha^{a_{1} t^{1}}+\alpha^{a_{2} t^{2}}+\alpha^{a_{3} t^{3}}+\ldots
$$

onde $a_{i} \in \mathbb{Z}$.

\section{Subgrupo fechado por estado e fecho por estado}

Um subgrupo $G$ de $\mathcal{A}_{m}$ é dito ser fechado por estado se para todo elemento $\alpha=\left(\alpha_{0}, \ldots, \alpha_{m-1}\right) \sigma(\alpha)$ de $G$ segue que $\alpha_{y}$ está em $G$, para todo $y=0, \ldots, m-1$. Em outros termos, $G$ é fechado por estado se para todo $\alpha \in G$ implica que $Q(\alpha)$ é um subconjunto de $G$. Se $G$ é um subgrupo de $\mathcal{A}_{m}$, denote por $\widehat{G}$ o fecho por estado de $G$, isto é, o subgrupo de $\mathcal{A}_{m}$ gerado por todos os estados de todos os elementos de $G$.

Exemplo 1.1.10. Seja $\alpha=(\alpha, \alpha \sigma)$ em $\mathcal{A}_{2}$, onde $\sigma=(01)$. Assim o grupo $\langle\alpha, \sigma\rangle$ é o fecho por estado de $\langle\alpha\rangle$. Em verdade, $\langle\alpha, \sigma\rangle$ é isomorfo ao Lamplighter Group $C_{2}>C$, onde o produto entrelaçado é restrito, $C_{2}$ é o cíclico de ordem 2 e $C$ é o cíclico infinito. Tal afirmação será demonstrada na próxima seção. 
Quando $Q(\alpha)$ é finito, para $\alpha \in \mathcal{A}_{m}$, então $\alpha$ é chamado de finito por estado. Se todo elemento de $G$ é finito por estado, então $G$ é chamado finito por estado. No Exemplo 1.1.10, o grupo $\langle\alpha, \sigma\rangle$ além de ser fechado por estado é finito por estado, pois é gerado pelos estados do autômato,

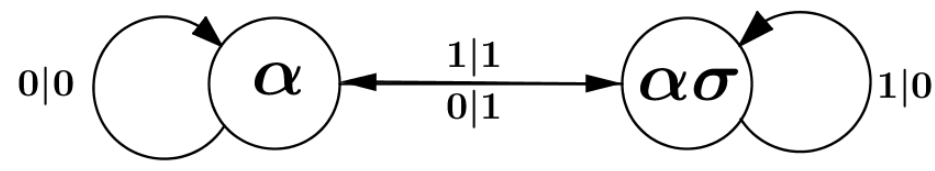

\section{Figura 1.8}

Vamos chamar um grupo transitivo e fechado por estado de recorrente se a projeção $\pi_{0}:$ Fix $x_{G}(0) \rightarrow G$ definida por

$$
\alpha^{\pi_{0}}=\left(\left(\alpha_{0}, \ldots, \alpha_{m-1}\right) \sigma(\alpha)\right)^{\pi_{0}}=\alpha_{0}
$$

é sobrejetora, onde $\alpha \in G$ e $0^{\sigma(\alpha)}=0$.

Com relação aos fechos topológico e diagonal, A. M. Brunner e S. N. Sidki demonstram que:

Proposição 1.1.11. ([3, página 459]) Seja A um grupo abeliano transitivo fechado por estado. Então os fechos diagonal e topológico comutam quando aplicados a A. Além disso, ofecho diagonal-topológico $A^{*}=\tilde{\bar{A}}$ é um grupo abeliano transitivo fechado por estado.

Mais que isso, eles mostram o seguinte resultado:

Teorema 1.1.12. ([3, página 463]) Seja A um grupo abeliano transitivo fechado por estado de grau $m$. Suponha que $A^{*}$ seja livre de torção. Então $A^{*}$ é um pro-m grupo finitamente gerado.

Esses geradores são determinados da seguinte forma: sejam $\sigma_{1}, \ldots, \sigma_{k} \in S_{m}$ tais que $P(A)=\left\langle\sigma_{1}, \ldots, \sigma_{k}\right\rangle$. Escolha $\alpha_{1}, \ldots, \alpha_{k} \in A$ tais que $\sigma\left(\alpha_{i}\right)=\sigma_{i}$, para todo $i=1, \ldots, k$. Então

$$
A^{*}=\left\langle\alpha_{1}, \ldots, \alpha_{k}\right\rangle^{*} .
$$

Para $A$ de torção eles mostram que:

Teorema 1.1.13. ([3, página 467]) Se A é um grupo abeliano transitivo fechado por estado de torção, então o expoente de Aé igual ao expoente de $P(A)$.

Para mais detalhes consulte [3]. 


\subsection{Representações como grupo de automorfismos da árvore}

\subsubsection{Representações de grau $m$}

Dizemos que um grupo abstrato $G$ possui uma representação de grau $m$ se existe um homomorfismo $\varphi: G \rightarrow \mathcal{A}_{m}$. Se $\varphi$ é monomorfismo, então tal representação é dita ser fiel. Se $G^{\varphi}$ é fechado por estado, transitivo ou finito por estado dizemos, repectivamente, que a representação é fechada por estado, transitiva ou finita por estado. Por vezes, chamaremos tanto $\varphi$ como $G^{\varphi}$ de representação de $G$ de grau $m$.

\section{Representação por classes laterais}

Sejam $G$ um grupo e $H_{0}, H_{1}, H_{2}, \ldots, H_{n}, \ldots$ subgrupos de $G$ tais que

$$
G=H_{0}>H_{1}>H_{2}>\ldots>H_{n}>\ldots
$$

e $\left[H_{i-1}: H_{i}\right]=m$, para todo $i>0$. Seja $T_{i}=\left\{t_{i, 0}, \ldots, t_{i, m-1}\right\}$, com $t_{i, 0}=1$, um transversal de $H_{i}$ em $H_{i-1}$. Então $G$ age naturalmente por multiplicação à direita sobre a árvore regular de classes laterais $T$ dada graficamente por:

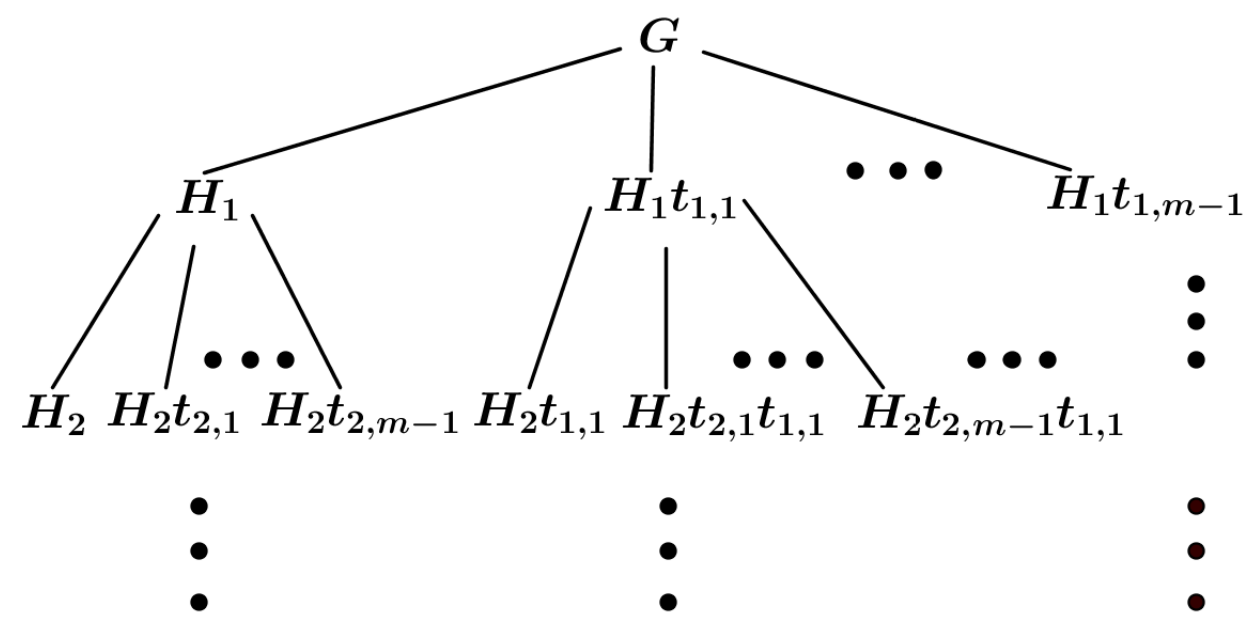

Figura 1.9: Árvore Regular de Classes Laterais. 
Como $\mathcal{T}$ é isomorfa a $\mathcal{T}_{m}$, como grafos, existe um homomorfismo natural $\varphi: G \rightarrow \mathcal{A}_{m}$. A representação $\varphi$ é transitiva, além disso, se não existe um subgrupo não trivial $K$ de $\cap_{i=0}^{\infty} H_{i}$ e normal em $G$, então $\varphi$ é fiel.

Exemplo 1.2.1. Seja $G$ um grupo abeliano livre de rank infinito livremente gerado por $x_{1}, x_{2}, x_{3}, x_{4} \ldots$. Considere a seguinte lista

$$
\begin{aligned}
& x_{1}^{2}, x_{1}^{4}, x_{1}^{8}, x_{1}^{16}, \ldots \\
& x_{2}^{2}, x_{2}^{4}, x_{2}^{8}, x_{2}^{16}, \ldots \\
& x_{3}^{2}, x_{3}^{4}, x_{3}^{8}, x_{3}^{16}, \ldots
\end{aligned}
$$

Utilizando um argumento parecido com o de enumeração dos racionais por Cantor, podemos considerar os seguintes subgrupos de $G$,

$$
\begin{gathered}
H_{1}=\left\langle x_{1}^{2}, x_{2}, x_{3}, x_{4} \ldots\right\rangle, H_{2}=\left\langle x_{1}^{4}, x_{2}, x_{3}, x_{4} \ldots\right\rangle, H_{3}=\left\langle x_{1}^{4}, x_{2}^{2}, x_{3}, x_{4} \ldots\right\rangle, \\
H_{4}=\left\langle x_{1}^{4}, x_{2}^{2}, x_{3}^{2}, x_{4} \ldots\right\rangle, H_{5}=\left\langle x_{1}^{4}, x_{2}^{4}, x_{3}^{2}, x_{4} \ldots\right\rangle, H_{6}=\left\langle x_{1}^{8}, x_{2}^{4}, x_{3}^{4}, x_{4} \ldots\right\rangle, \\
H_{7}=\left\langle x_{1}^{16}, x_{2}^{4}, x_{3}^{4}, x_{4} \ldots\right\rangle, H_{5}=\left\langle x_{1}^{16}, x_{2}^{8}, x_{3}^{4}, x_{4}^{2}, x_{5}, \ldots\right\rangle, \ldots, H_{n}, \ldots
\end{gathered}
$$

Note que $G>H_{1}>H_{2}>\ldots>H_{n}>\ldots,\left[H_{i+1}: H_{i}\right]=2$, para todo $i=$ $1,2, \ldots, n, \ldots, e \cap_{i=1}^{\infty} H_{i}=\{1\}$. Portanto, existe uma representação fiel de $G$ em $\mathcal{A}_{2}$. Na verdade, com o mesmo processo, existe um representação fiel de $G$ em $\mathcal{A}_{m}$, qualquer que seja m maior ou igual a 2.

\subsubsection{Representações fechadas por estado}

As representações fechadas por estado são, neste trabalho, as representações de maior interesse.

Sejam $G$ um grupo, $H$ um subgrupo de índice $m$ em $G$ e $f: H \rightarrow G$ um homomorfismo (também chamado de endomorfismo virtual). Escolha $T=$ $\left\{t_{0}, t_{1}, \ldots, t_{m-1}\right\}$ um transversal de $H$ em $G$. Agora, cada $g$ em $G$ induz uma permutação $\sigma(g): Y \rightarrow Y$ com relação a $T$, dada por

$$
i^{\sigma(g)}=j \Leftrightarrow H t_{i} g=H t_{j}, i, j=0, \ldots, m-1 .
$$

Note que $t_{i} g t_{j}^{-1} \in H$, ou seja, $t_{i} g t_{i^{\sigma(g)}}^{-1} \in H$. Defina $\varphi: G \rightarrow \mathcal{A}_{m}$ por

$$
g \mapsto\left(\left[t_{0} g t_{0^{\sigma(g)}}^{-1}\right]^{f \varphi},\left[t_{1} g t_{1^{\sigma(g)}}^{-1}\right]^{f \varphi}, \ldots,\left[t_{m-1} g t_{(m-1)^{\sigma(g)}}^{-1}\right]^{f \varphi}\right) \sigma(g) .
$$

Claramente $\varphi$ é uma função bem definida. 
Proposição 1.2.2. ([19, página 8]) A função $\varphi$ definida acima é um homomorfismo, onde $G^{\varphi}$ é fechado por estado, transitivo e

$$
\operatorname{ker}(\varphi)=\left\langle K \leqslant H \mid K \triangleleft G, K^{f} \leqslant K\right\rangle,
$$

chamado o $f-\operatorname{core}(H)$.

Demonstração. A demonstração é feita por indução sobre o comprimento $|u|$ de um vértice $u \in \mathcal{T}_{m}$. Sejam $g, h \in G$. Temos, por um lado,

$$
\begin{gathered}
(g h)^{\varphi}=\left(\left[t_{0} g h t_{0^{\sigma(g h)}}^{-1}\right]^{f \varphi}, \ldots,\left[t_{m-1} g h t_{(m-1)^{\sigma(g h)}}^{-1}\right]^{f \varphi}\right) \sigma(g h)= \\
=\left(\left[t_{0} g t_{0^{\sigma(g)}}^{-1} t_{0^{\sigma(g)}} h t_{0^{\sigma(g h)}}^{-1}\right]^{f \varphi}, \ldots,\left[t_{m-1} g t_{(m-1)^{\sigma(g)}}^{-1} t_{(m-1)^{\sigma(g)}} h t_{(m-1)^{\sigma(g h)}}^{-1}\right]^{f \varphi}\right) \sigma(g h) .
\end{gathered}
$$

E por outro

$$
\begin{aligned}
g^{\varphi} h^{\varphi} & =\left(\left[t_{i} g t_{i^{\sigma(g)}}^{-1}\right]^{f \varphi}\right)_{i \in Y} \sigma(g) \cdot\left(\left[t_{i} h t_{i^{\sigma(h)}}^{-1}\right]^{f \varphi}\right)_{i \in Y} \sigma(h)= \\
= & \left(\left[t_{i} g t_{i^{\sigma(g)}}^{-1}\right]^{f \varphi}\left[t_{i^{\sigma(g)}} h t_{i^{\sigma(h) \sigma(g)}}^{-1}\right]^{f \varphi}\right)_{i \in Y} \sigma(g) \sigma(h) .
\end{aligned}
$$

É fácil ver que $\sigma(g h)=\sigma(g) \sigma(h)$. Assim, para todo $i \in Y$ segue que

$$
i^{(g h)^{\varphi}}=i^{\sigma(g h)}=i^{\sigma(g) \sigma(h)}=i^{g^{\varphi} h^{\varphi}} .
$$

Suponha por indução que o resultado vale para toda palavra com comprimento menor ou igual que $k$. Assim para todo $i \in Y$ e toda parlavra $u$ de comprimento $k$ vale que

$$
(i u)^{(g h)^{\varphi}}=i^{\sigma(g h)} u^{(g h)_{i}^{\varphi}},(i u)^{g^{\varphi} h^{\varphi}}=i^{\sigma(g h)} u^{\left(g^{\varphi} h^{\varphi}\right)_{i}} .
$$

Por hipótese de indução, temos

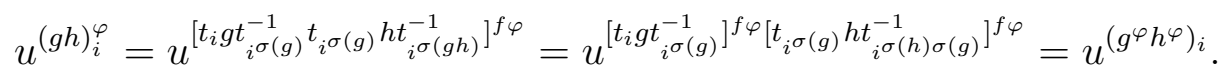

Segue que $\varphi$ é homomorfismo. As outras afirmações são imediatas.

Se $f-\operatorname{core}(H)=1$, dizemos que $f$ é simples, ou seja, $G \simeq G^{\varphi}$ e a representação é fiel.

Exemplo 1.2.3. Seja $G=C_{2} \imath C=\langle a\rangle \curlywedge\langle x\rangle$. Então $A=\operatorname{Tor}(G)=\langle a\rangle^{\langle x\rangle} e$ $G^{\prime}=\left\langle a a^{x}\right\rangle^{\langle x\rangle}$. Considere $H=G^{\prime}\langle x\rangle$ e $T=\{1, a\}$ um transversal de $H$ em $G$ (note que $[G: H]=2$ ). Não é difícil ver que $H \simeq G$ e $f: H \rightarrow G$ o homomorfismo que estende a função

$$
a a^{x} \mapsto a, x \mapsto x
$$


é um isomorfismo. Agora, se $K$ é um subgrupo não trivial de $H$, normal em $G$ e f-invariante, então $K \cap A$ também o é, pois nenhum subgrupo livre de torção satisfaz tais propriedades. Como $K \cap A \leqslant G^{\prime} \cap A=G^{\prime}$, segue que $K \cap A$ é trivial, pois $f$ diminui pela metade o comprimento de uma palavra não trivial em $\left\{\left(a a^{x}\right)^{y} ; y \in\langle x\rangle\right\}$. Isso mostra que $f$ é simples $e G \simeq G^{\varphi}$. Como

$$
a^{\varphi}=(01), x^{\varphi}=\left(x^{\varphi}, x^{\varphi} a^{\varphi}\right)
$$

segue que

$$
G \simeq\langle\alpha=(\alpha, \alpha \sigma), \sigma=(01)\rangle,
$$

como no Exemplo 1.1.10.

Claramente o homomorfismo $\varphi$ depende do transversal escolhido, $\log 0 \varphi=$ $\varphi_{T}$. Abaixo, uma proposição que mostra que numa outra escolha de um transvesal de $H$ em $G$ é produzida uma representação de $G$ conjugada com a original por um elemento bem determinado de $\mathcal{A}_{m}$.

Proposição 1.2.4. ([3, página 464]) Sejam

$$
T=\left\{t_{0}, \ldots, t_{m-1}\right\}, T^{\prime}=\left\{t_{0}^{\prime}=h_{0} t_{0}, \ldots, t_{m-1}^{\prime}=h_{m-1} t_{m-1}\right\}
$$

dois transversais de $H$ em $G$, onde $h_{0}, \ldots, h_{m-1} \in H$. Sejam $\varphi_{T}$ e $\varphi_{T^{\prime}}$, como acima. Então existe $\beta \in \mathcal{A}_{m}$ tal que

$$
g^{\varphi_{T}}=\beta^{-1} g^{\varphi_{T^{\prime}} \beta}
$$

para todo $g \in G$.

Demonstração. Sejam $\varphi=\varphi_{T}$ e $\varphi^{\prime}=\varphi_{T^{\prime}}$. Assim

$$
g^{\varphi}=\left(\left[t_{0} g t_{0^{\sigma(g)}}^{-1}\right]^{f \varphi},\left[t_{1} g t_{1^{\sigma(g)}}^{-1}\right]^{f \varphi}, \ldots,\left[t_{m-1} g t_{(m-1)^{\sigma(g)}}^{-1}\right]^{f \varphi}\right) \sigma(g)
$$

$\mathrm{e}$

$$
g^{\varphi^{\prime}}=\left(\left[t_{0}^{\prime} g\left(t_{0^{\sigma(g)}}^{\prime}\right)^{-1}\right]^{f \varphi},\left[t_{1}^{\prime} g\left(t_{1 \sigma(g)}^{\prime}\right)^{-1}\right]^{f \varphi^{\prime}}, \ldots,\left[t_{m-1}^{\prime} g\left(t_{(m-1)^{\sigma(g)}}^{\prime}\right)^{-1}\right]^{f \varphi^{\prime}}\right) \sigma(g),
$$

para todo $g \in G$. Daí

$$
\begin{gathered}
g^{\varphi^{\prime}}=\left(\left[t_{0}^{\prime} g\left(t_{0^{\sigma(g)}}^{\prime}\right)^{-1}\right]^{f \varphi}, \ldots,\left[t_{m-1}^{\prime} g\left(t_{(m-1)^{\sigma(g)}}^{\prime}\right)^{-1}\right]^{f \varphi^{\prime}}\right) \sigma(g)= \\
=\left(\left[h_{0} t_{0} g\left(h_{0^{\sigma(g)}} t_{0^{\sigma(g)}}\right)^{-1}\right]^{f \varphi}, \ldots,\left[h_{m-1} t_{m-1} g\left(h_{(m-1)^{\sigma(g)}} t_{(m-1)^{\sigma(g)}}\right)^{-1}\right]^{f \varphi^{\prime}}\right) \sigma(g)=
\end{gathered}
$$




$$
=\left(h_{0}^{f \varphi^{\prime}}, \ldots, h_{m-1}^{f \varphi^{\prime}}\right)\left(\left[t_{0} g t_{0^{\sigma(g)}}^{-1}\right]^{f \varphi^{\prime}}, \ldots,\left[t_{m-1} g t_{(m-1)^{\sigma(g)}}^{-1}\right]^{f \varphi^{\prime}}\right) \sigma(g)\left(h_{0}^{f \varphi^{\prime}}, \ldots, h_{m-1}^{f \varphi^{\prime}}\right)^{-1}
$$

onde o automorfismo $\beta_{0}=\left(h_{0}^{f \varphi^{\prime}}, \ldots, h_{m-1}^{f \varphi^{\prime}}\right)^{-1}$ é independente de $g$. Replicando o processo para cada $g_{i}=\left(t_{i} g t_{i^{\sigma(g)}}^{-1}\right)^{f \varphi}$, vamos obter no infinito

$$
g^{\varphi^{\prime}}=\beta^{-1} g^{\varphi} \beta
$$

onde $\beta=\beta_{0} \beta_{1} \ldots \beta_{n} \ldots$ e $\beta_{n+1}=\beta_{n}^{(n+1)}$, com $n$ inteiro não negativo.

Exemplo 1.2.5. Considere a representação $\varphi$ de $G=C_{2} \curlywedge C$, do Exemplo 1.2.3. Assim $G^{\varphi}=\langle\alpha=(\alpha, \alpha \sigma), \sigma=(01)\rangle$. Escolha o transversal $T^{\prime}=\left\{1, a^{x}\right\}=$ $\left\{1, a a^{x} a\right\}$. Como $\left(a a^{x}\right)^{f}=a$, então

$$
\beta_{0}=\left(e, a^{\varphi^{\prime}}\right)^{-1}=(e, \gamma)
$$

onde $\gamma=(\gamma, \gamma) \sigma$. Note que $\sigma^{\alpha^{-1}}=\left(\sigma^{\alpha^{-1}}, \sigma^{\alpha^{-1}}\right) \sigma$, ou seja, $\gamma=\sigma^{\alpha^{-1}}$. Daí $\beta=(e, \gamma)(e, \gamma)^{(1)}(e, \gamma)^{(2)} \ldots=(\beta, \gamma \beta) e$

$$
a^{\varphi^{\prime}}=\sigma^{\beta}=\sigma^{\alpha^{-1}}, x^{\varphi^{\prime}}=\alpha^{\beta}=\alpha,
$$

pois $\alpha$ e $\beta$ comutam.

Proposição 1.2.6. Um grupo $G$ é um grupo transitivo fechado por estado de grau $m$ se, e somente se, existem um subgrupo $H$ de indice $m$ em $G$ e $f: H \rightarrow G$ um endomorfismo simples.

Demonstração. A recíproca segue da Proposição 1.2.2. Agora se $G$ é um subgrupo transitivo e fechado por estado, então

$$
\begin{aligned}
\pi_{0}: \operatorname{Fix}_{G}(0) & \rightarrow G \\
\alpha & \mapsto \alpha_{0}
\end{aligned}
$$

é um endomorfismo simples e $\left[G: F i x_{G}(0)\right]=m$.

Em particular, fica demonstrado que se $A$ é um autômato, então $\mathcal{G}(A)$ induz um endomorfismo simples $f: F i x_{\mathcal{G}(A)}(0) \rightarrow \mathcal{G}(A)$. Mas será que dado um autômato $A$ sobre um alfabeto com $m$ letras existem um subgrupo $H$ de $\mathcal{G}(A)$ e um endomorfismo $f: H \rightarrow \mathcal{G}(A)$ que induzem os estados de $A$ ? Induz no sentido que $(\mathcal{G}(A))^{\varphi}=\mathcal{G}(A)$. A resposta a essa pergunta é negativa, como mostra a proposição. 
Proposição 1.2.7. Dado o autômato A,

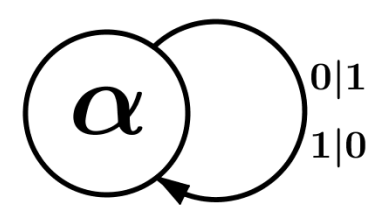

Figura 1.10

não existem um subgrupo $H$ de índice dois de $\mathcal{G}(A)$ e um endomorfismo simples $f: H \rightarrow \mathcal{G}(A)$ tais que $(\mathcal{G}(A))^{\varphi}=\mathcal{G}(A)$, onde $\varphi$ é uma representação induzida porf e $H$.

Demonstração. Note que

$$
\mathcal{G}(A)=\langle\alpha=(\alpha, \alpha)(01)\rangle
$$

$\mathrm{e}$

$$
\alpha^{2}=\left(\alpha^{2}, \alpha^{2}\right)=e
$$

ou seja, $\mathcal{G}(A)$ é uma representação transitiva e fechada por estado de $C_{2}=\langle a\rangle$. Por outro lado, as únicas possibilidades para

i) um subgrupo $H$ de $G$ de índice dois;

ii) um endomorfismo simples $f: H \rightarrow G$ e

iii) um transversal $T$ de $H$ em $G$

são $H=\{1\}, f$ o homomorfismo que leva 1 em 1 e $T=\{1, a\}$. Portanto, a única representação $\varphi: C_{2} \rightarrow \mathcal{A}_{2}$ transitiva e fechada por estado de grau dois induzida por um endomorfismo simples é tal que

$$
a^{\varphi}=\left((1 a a)^{f \varphi},(a a 1)^{f \varphi}\right)(01)=(01),
$$

que é diferente da representação inicial.

Quando não houver confusão chamaremos uma representação tansitiva fechada por estado de apenas representação fechada por estado. 
CAPÍTULO 2

\section{Endomorfismos de grupos metabelianos tipo entrelaçado}

Neste capítulo analisamos endomorfismos virtuais de grupos metabelianos tipo entrelaçado. Com isso estamos querendo dizer que analisamos endomorfismos virtuais de um produto entrelaçado restrito $G=B \imath X$, onde $B$ e $X$ são abelianos.

\subsection{Endomorfismos de produtos semidiretos}

Dizemos que um grupo $K$ é metabeliano se seu subgrupo derivado $K^{\prime}$ é abeliano. Agora, um grupo $G$ é dito ser um produto semidireto do subgrupo $A$ pelo subgrupo $X$ se $G=A X, A$ é normal em $G$ e $A \cap X=\{1\}$. Vamos supor que existem $H$ um subgrupo de índice finito $m$ em $G$ e um endomorfismo $f: H \rightarrow G$. Defina $Y=A H \cap X, A_{0}=A \cap H$ e $\dot{H}=A_{0} Y$. Vamos supor também que $A_{0}$ é normal em $G$.

Proposição 2.1.1. Nessas condições, o índice de $\dot{H}$ em Gé igual a m.

Demonstração. Note que $m=[G: H]=[G: A H][A H: H]$ e $[A H: H]=[A$ : $A \cap H], \log 0 m_{1}=\left[A: A_{0}\right]$ divide $m$. Analogamente, $m_{2}=[X: Y]$ divide $m$. Afirmamos que $m=m_{1} m_{2}$. De fato, sejam $S$ e $T$ transversais, respectivamente, de $A_{0}$ em $A$ e de $Y$ em $X$. Pondo $H=A_{0}\langle v(y) y \mid y \in Y\rangle$, onde $v(y) \in A$ para 
todo $y \in Y$, temos

$$
\begin{gathered}
H S T=A_{0}\langle v(y) y \mid y \in Y\rangle S T=\langle v(y) y \mid y \in Y\rangle\left(A_{0} S\right) T= \\
=\langle v(y) y \mid y \in Y\rangle A T=A\langle v(y) y \mid y \in Y\rangle T=A(Y T)=A X=G .
\end{gathered}
$$

Disto, segue que $S T$ é um transversal de $H$ em $G$ e $m=m_{1} m_{2}$.

Como $A_{0}$ é normal em $G$ segue que é normal em $A H$, daí

$$
\dot{H} S T=A_{0} Y S T=Y A_{0} S T=Y A T=A Y T=A X=G
$$

Portanto, $S T$ é um transversal de $\dot{H}$ em $G$. O resultado segue.

Suponha que $[A, Y]$ é subgrupo de $A_{0}$ e $H$ é normal. Então $Y$ é normal em $X$, assim para todo $g=a x \in G$ temos

$$
\left(a_{0} y\right)^{g}=\left(a_{0} y\right)^{a x}=a_{0}^{g}\left[a^{x}, y^{-x}\right] y^{x} \in A_{0} Y,
$$

para todo $a_{0} \in A_{0}$ e todo $y \in Y$. Logo $A_{0} Y$ é normal em $G$ e podemos enunciar a proposição:

Proposição 2.1.2. Se $H$ é normal em $G$ e $[A, Y]$ é subgrupo de $A_{0}$, então $\dot{H}$ é normal em $G$.

Como corolário temos:

Corolário 2.1.3. Se A é abeliano e $H$ é normal em $G$, então $\dot{H}$ é normal em $G$.

Demonstração. Se $A$ é abeliano, então para todos $a, b \in A$ e $y \in Y$ temos

$$
[a, y]=[a, b y]
$$

ou seja, $[A, Y]=[A, A Y]=[A, H]$. Pela Proposição 2.1.2, segue que $\dot{H}$ é normal.

Vamos analisar agora o caso onde o homomorfismo $f: H \rightarrow G$ é tal que $A_{0}^{f}$ é subgrupo de $A$. São inúmeros os casos onde isso ocorre, por exemplo, basta que $A$ seja de torção e $X$ livre de torção.

Nesse caso, definimos $\mu: A_{0} \rightarrow A$ por $a_{0}^{\mu}=a_{0}^{f}$. Claramente $\mu$ é um homomorfismo. Também definimos $\alpha: Y \rightarrow X$ pondo $y^{\alpha}=y^{\prime}$, onde $y^{\prime}$ é obtido de $(a y)^{f}=b y^{\prime}$, onde $a, b \in A$. Afirmamos que $\alpha$ é um homomorfismo bem definido. 
De fato, se $a_{1}, a_{2}, b_{1}, b_{2} \in A$ e $y^{\prime}, y^{\prime \prime} \in Y$, com $y^{\prime}$ diferente de $y^{\prime \prime}$, são tais que $\left(a_{1} y\right)^{f}=b_{1} y^{\prime}$ e $\left(a_{2} y\right)^{f}=b_{2} y^{\prime \prime}$, então $\left(a_{1} y\right)^{f}\left(a_{2} y\right)^{-f}=\left(a_{1} a_{2}^{-1}\right)^{f} \in A$, mas

$$
\left(a_{1} y\right)^{f}\left(a_{2} y\right)^{-f}=b_{1} y^{\prime}\left(y^{\prime \prime}\right)^{-1} b_{2}^{-1}=b_{1} b_{2}^{-y^{\prime \prime}\left(y^{\prime}\right)^{-1}} y^{\prime}\left(y^{\prime \prime}\right)^{-1} .
$$

Ou seja, $y^{\prime}=y^{\prime \prime}$, absurdo. Logo $\alpha$ está bem definido. Que $\alpha$ é homomorfismo é claro. Se $A$ é abeliano, vamos definir um homomorfismo $\dot{f}$ de $\dot{H}$ em $G$ que em certo sentido é mais simples que $f$, pondo

$$
\begin{aligned}
\dot{f}: \dot{H}\left(=A_{0} Y\right) & \rightarrow G \\
a_{0} y & \mapsto a_{0}^{\mu} y^{\alpha}
\end{aligned}
$$

para todo $a_{0} \in A_{0}$ e todo $y \in Y$. Pela observação acima, $\dot{f}$ é uma função.

Proposição 2.1.4. Se A é abeliano, então $\dot{f}$ é um homomorfismo.

Demonstração. Para mostrar que $\dot{f}$ é homomorfismo, precisamos provar que para quaisquer $a_{1}, a_{2} \in A_{0}$ e quaisquer $y_{1}, y_{2} \in Y$ temos que

$$
\left(a_{1} y_{1} a_{2} y_{2}\right)^{\dot{f}}=\left(a_{1} a_{2}^{y_{1}^{-1}} y_{1} y_{2}\right)^{\dot{f}}=a_{1}^{\mu}\left(a_{2}^{y_{1}^{-1}}\right)^{\mu} y_{1}^{\alpha} y_{2}^{\alpha}
$$

$\mathrm{e}$

$$
\left(a_{1} y_{1}\right)^{\dot{f}}\left(a_{2} y_{2}\right)^{\dot{f}}=a_{1}^{\mu} y_{1}^{\alpha} a_{2}^{\mu} y_{2}^{\alpha}=a_{1}^{\mu}\left(a_{2}^{\mu}\right)^{y_{1}^{-\alpha}} y_{1}^{\alpha} y_{2}^{\alpha}
$$

são iguais. Ou seja, devemos provar que para quaisquer $a_{0} \in A_{0}$ e $y \in Y$ temos $\left(a_{0}^{y}\right)^{\mu}=\left(a_{0}^{\mu}\right)^{y^{\alpha}}$. Com efeito, sejam $b, c \in A$ tais que $b y \in H$ e $(b y)^{f}=c y^{\alpha}$, então

$$
\left(a_{0}^{y}\right)^{\mu}=\left(a_{0}^{b y}\right)^{f}=\left(a_{0}^{f}\right)^{(b y)^{f}}=\left(a_{0}^{\mu}\right)^{c y^{\alpha}}=\left(a_{0}^{\mu}\right)^{y^{\alpha}} .
$$

Como desejado.

E quanto a simplicidade, se $f$ é simples, implica que sendo $\dot{f}$ um homomorfismo ele é simples? Uma resposta parcial à essa pergunta é a seguinte.

Proposição 2.1.5. Se A é abeliano, $C_{X}(A)=\{1\}$ e $f$ é simples, então $\dot{f}$ é simples.

Demonstração. Seja $K$ um subgrupo de $\dot{H}$, normal em $G$ e $f$-invariante. Considere $K_{0}=K \cap A_{0}$. Assim

$$
K_{0}^{\dot{f}}=\left(K \cap A_{0}\right)^{\dot{f}} \leqslant K^{\dot{f}} \cap A \leqslant K \cap A=(K \cap H) \cap A=K \cap(H \cap A)=K_{0} .
$$

Mas $K_{0}^{\dot{f}}=K_{0}^{f}$, donde $K_{0}$ é trivial. Logo $[K, A] \leqslant K_{0}=\{1\}$, ou seja, $K \leqslant$ $C_{X}(A)=\{1\}$. Portanto, $\dot{f}$ é simples. 
Exemplo 2.1.6. Considere o grupo $G=B \nmid C$, com $B$ abeliano finito, e $H$ um subgrupo de índice finito $m$ em $G$. Suponha que $H \cap \operatorname{Tor}(G)$ é normal em $G$ e tome $A=\operatorname{Tor}(G)=\oplus_{x \in C} B, X=C=\langle x\rangle, Y=A H \cap X=\left\langle x^{r}\right\rangle e$ $A_{0}=A \cap H$. Pela Proposição 2.1.5, $H$ pode ser replicado por $A_{0} Y$ e a ação de $f$ pode ser dada por

$$
\left(a_{0} x^{k r}\right)^{f}=a_{0}^{f} x^{k s}
$$

onde $a_{0}^{f} \in A$ e $x^{r f}=x^{s}$, para quaisquer $a_{0} \in A_{0}$ e $k$ inteiro. Se $[G: H]=2$, os endomorfismos simples podem ser considerados como acima, pois $H \cap T$ Tor $(G) e ́$ normal em $G$.

Segue da Proposição 2.1.5 o seguinte corolário que será usado na próxima seção.

Corolário 2.1.7. Nas hipóteses da Proposição 2.1.5, com $[G: H]=p$ primo, podemos supor que $X \leqslant H$.

Demonstração. De fato, podemos considerar $H=A_{0} Y$. Como $[G: H]$ é primo, devemos ter $\left[A: A_{0}\right]=p$ ou 1 . Se for o segundo caso, $A$ é subgrupo de $H$, o que não é possível, pois $f$ é simples, daí $\left[A: A_{0}\right]=p$. Portanto, $Y=X$.

Suponha que $Y=X$. Seja $K$ um subgrupo de $A_{0}$ normal em $A$ e $f$-invariante. Logo $K^{X}$ é um subgrupo de $A_{0}$ normal de $G$ e $f$-invariante, pois

$$
\left(K^{X}\right)^{f}=\left(K^{f}\right)^{X^{f}} \leqslant K^{X} .
$$

Daí $K=\{1\}$. Assim, temos a seguinte proposição:

Proposição 2.1.8. Dadas as hipóteses acima, segue que $f: A_{0} \rightarrow$ A é um endomorfismo simples.

Note que se $X$ é um subgrupo de $H$, segue que $A_{0}$ é normal em $G$.

\subsection{Endomorfismos de grupos tipo Lamplighter}

Seja $B$ um grupo abeliano finito. Então $B$ é tal que

$$
B=\left\langle b_{1}\right\rangle \oplus \ldots \oplus\left\langle b_{r}\right\rangle,
$$

com $\left\langle b_{i}\right\rangle$ cíclico de ordem $n_{i}$. Definimos $G_{B, d}$ como o produto entrelaçado restrito de $B$ por $C^{d}$, isto é, $G_{B, d}=B \imath C^{d}$. Ao grupo $G_{B, d}$ chamaremos de grupo tipo Lamplighter. 
Podemos olhar para $G_{B, d}$ como o produto semidireto $A X$, onde $A=\oplus_{x \in X} B$ e $X=C^{d}=\left\langle x_{1}, \ldots, x_{d}\right\rangle$. Assim, cada elemento de $G_{B, d}$ tem a forma geral

$$
\left(b_{1}^{p_{1}\left(x_{1}, \ldots, x_{d}\right)} \ldots b_{r}^{p_{r}\left(x_{1}, \ldots, x_{d}\right)}\right)\left(x_{1}^{s_{1}} \ldots x_{d}^{s_{d}}\right),
$$

onde $p_{i}\left(x_{1}, \ldots, x_{d}\right)$ é um elemento de $\left(\mathbb{Z} / n_{i} \mathbb{Z}\right)\langle X\rangle$, para todo $i=1, \ldots, r$, ou seja, $p_{i}\left(x_{1}, \ldots, x_{d}\right)$ é um polinômio, com expoentes podendo ser negativos, sobre o anel $\mathbb{Z} / n_{i} \mathbb{Z}$. Temos ainda

$$
\left(b_{1}^{p_{1}\left(x_{1}, \ldots, x_{d}\right)} \ldots b_{r}^{p_{r}\left(x_{1}, \ldots, x_{d}\right)}\right) x_{i}^{s_{i}}=x_{i}^{s_{i}}\left(b_{1}^{p_{1}\left(x_{1}, \ldots, x_{d}\right) x_{i}^{s_{i}}} \ldots b_{r}^{p_{r}\left(x_{1}, \ldots, x_{d}\right) x_{i}^{s_{i}}}\right),
$$

para todo $i=1, . ., r$.

Tome $r=1, B=\langle b\rangle$, com $|b|=n$ e denote $G_{B, d}$ por $G_{n, d}$. Suponha que $f: H \rightarrow G_{n, d}$ é um endomorfismo simples e $H$ é um subgrupo de índice $m$ em $G_{n, d}$ tal que $A_{0}=H \cap A$ é normal em $G_{n, d}$. Temos que $A$ é abeliano e $C_{X}(A)=\{1\}$. Pela Proposição 2.1.5, dado um elemento $b^{p\left(x_{1}, \ldots, x_{d}\right)} x_{1}^{r_{1}} \ldots x_{d}^{r_{d}}$ de $H$, a ação de $f$ pode ser dada por

$$
\left(b^{p\left(x_{1}, \ldots, x_{d}\right)} x_{1}^{r_{1}} \ldots x_{d}^{r_{d}}\right)^{f}=b^{\left(p\left(x_{1}, \ldots, x_{d}\right)\right)^{\mu}}\left(x_{1}^{r_{1}} \ldots x_{d}^{r_{d}}\right)^{\alpha},
$$

onde $Y=A H \cap X, \alpha: Y \rightarrow X$ é um homomorfismo e $\mu: \mathcal{I} \rightarrow \mathcal{A}$ é um homomorfismo de grupos abelianos entre os anéis $\mathcal{I}$ e $\mathcal{A}=(\mathbb{Z} / n \mathbb{Z})\langle X\rangle$, com $\mathcal{I}$ um ideal de $\mathcal{A}$. De fato, temos que $A$ pode ser visto como o anel de grupo $(\mathbb{Z} / n \mathbb{Z})\langle X\rangle$ e $A_{0}$ como um ideal de $(\mathbb{Z} / n \mathbb{Z})\langle X\rangle$, pois é normal em $G_{n, d}$. Assim $f: A_{0} \rightarrow A$ é o homomorfismo $\mu: \mathcal{I} \rightarrow \mathcal{A}$. Note que $\alpha$ se estende a um homomorfismo de grupos abelianos $\alpha: \mathcal{B} \rightarrow \mathcal{A}$, onde $\mathcal{B}=(\mathbb{Z} / n \mathbb{Z})\langle Y\rangle$. Se $p \in \mathcal{I}$ e $q \in \mathcal{B}$, então $\left(b^{p q}\right)^{\mu}=b^{p^{\mu} q^{\alpha}}$.

Se $K=\operatorname{ker}_{\mathcal{B}}(\alpha)$, então $\mathcal{I} K$ é um ideal de $\mathcal{A}$ contido em $\operatorname{ker}_{\mathcal{I}}(\mu), \operatorname{logo}$ o subgrupo $\left\langle b^{\mathcal{I} K}\right\rangle$ é um subgrupo de $H$, normal em $G$ e $f$-invariante e, assim, trivial se $f$ é simples, neste caso, $\alpha$ é um monomorfismo.

Com essas observações e pela Proposição 2.1.5, temos a proposição:

Proposição 2.2.1. Seja $f: H \rightarrow G_{n, d}$ um endomorfismo, com $H$ um subgrupo de indice finito em $G_{n, d}$. Então $f$ é simples se, e somente se, o único ideal de $\mathcal{A}$ contido em $\mathcal{I}$ e $\mu$-invariante é o ideal trivial $\{0\}$. Além disso, $f$ simples implica que $\alpha$ é um monomorfismo.

Vamos aplicar essa proposição para mostrar que $G_{n, 2}=C_{n} \prec C^{2}$ pode ser representado como um grupo de automorfismos fechado por estado de grau $n^{n+2}$, ou seja, vamos mostrar que existe um endomorfismo simples $f: H \rightarrow G_{n, 2}$ tal que $\left[G_{n, 2}: H\right]=n^{n+2}$. 
Exemplo 2.2.2. Considere $G_{n, 2}=C_{n} \curlywedge C^{2}=\langle a\rangle \curlywedge\langle x, y\rangle$. Seja

$$
H=\left\langle a^{x^{n}-1}, a^{y-1}\right\rangle^{\langle x, y\rangle}\left\langle x^{n}, y\right\rangle .
$$

Éfácil ver que $\left[G_{n, 2}: H\right]=n^{n+2}$ e $H \cap A$ é normal em $G_{n, 2}$, onde $A=\operatorname{Tor}\left(G_{n, 2}\right)$. Pela Proposição 2.1.5, se existe um endomorfismo simples $f: H \rightarrow G_{n, 2}$, então podemos considerar $f$ tal que

$$
\left(\left\langle a^{x^{n}-1}, a^{y-1}\right\rangle^{\langle x, y\rangle}\right)^{f} \leqslant A,\left\langle x^{n}, y\right\rangle^{f} \leqslant\langle x, y\rangle .
$$

Vamos usar a notação da Proposição 2.2.1 e determinar $\mu$ e $\alpha$ de tal modo que $f$ fique bem definida e seja um endomorfismo simples. Veja que $\mathcal{A}$ é o anel de todos os polinômios de duas variáveis $x$ e y sobre o anel $\mathbb{Z} / n \mathbb{Z}$ com expoentes em $\mathbb{Z}$, isto é, $\mathcal{A}=(\mathbb{Z} / n \mathbb{Z})\langle x, y\rangle$. Veja, também, que o ideal $\mathcal{I}$ é o ideal de $\mathcal{A}$ gerado pelos polinômios $x^{n}-1$ e $y-1$. Escreva $\langle x, y\rangle=\oplus_{i=0}^{n-1} x^{i}\left\langle x^{n}, y\right\rangle$, assim

$$
\mathcal{A}=(\mathbb{Z} / n \mathbb{Z})\langle x, y\rangle=\bigoplus_{i=0}^{n-1}(\mathbb{Z} / n \mathbb{Z})\left\langle x^{n}, y\right\rangle x^{i}
$$

$e$

$$
\begin{gathered}
\mathcal{I}=(\mathbb{Z} / n \mathbb{Z})\langle x, y\rangle\left(x^{n}-1\right)+(\mathbb{Z} / n \mathbb{Z})\langle x, y\rangle(y-1)= \\
=\sum_{i=0}^{n-1}(\mathbb{Z} / n \mathbb{Z})\left\langle x^{n}, y\right\rangle x^{i}\left(x^{n}-1\right)+\sum_{i=0}^{n-1}(\mathbb{Z} / n \mathbb{Z})\left\langle x^{n}, y\right\rangle x^{i}(y-1) .
\end{gathered}
$$

Se $r(x, y) \in \mathcal{A}$, podemos escrever

$$
r(x, y)=s(1, y)+s^{\prime}(x, y)(x-1)=r(x, 1)+r^{\prime}(x, y)(y-1),
$$

assim, se $r(x, y) \in \mathcal{I}$, então

$$
\begin{aligned}
r(x, y) & =\sum_{i=0}^{n-1} r_{i}\left(x^{n}, y\right) x^{i}\left(x^{n}-1\right)+\sum_{i=0}^{n-1} s_{i}\left(x^{n}, y\right) x^{i}(y-1)= \\
& =\sum_{i=0}^{n-1}\left(r_{i}\left(x^{n}, 1\right)+r_{i}^{\prime}\left(x^{n}, y\right)(y-1)\right) x^{i}\left(x^{n}-1\right)+ \\
& +\sum_{i=0}^{n-1}\left(s_{i}(1, y)+s_{i}^{\prime}\left(x^{n}, y\right)\left(x^{n}-1\right)\right) x^{i}(y-1)=
\end{aligned}
$$




$$
\begin{aligned}
= & \sum_{i=0}^{n-1} r_{i}\left(x^{n}, 1\right) x^{i}\left(x^{n}-1\right)+\sum_{i=0}^{n-1} s_{i}(1, y) x^{i}(y-1)+ \\
& +\sum_{i=0}^{n-1}\left(r_{i}^{\prime}\left(x^{n}, y\right)+s_{i}^{\prime}\left(x^{n}, y\right)\right) x^{i}\left(x^{n}-1\right)(y-1) .
\end{aligned}
$$

Essa escrita é única. Defina $\mu: \mathcal{I} \rightarrow \mathcal{A}$ por

$$
\begin{aligned}
(r(x, y))^{\mu} & =\sum_{i=0}^{n-1} r_{i}(y, 1) x^{i}(y-1)+\sum_{i=0}^{n-1} s_{i}(1, x) x^{i}(x-1)+ \\
+ & \sum_{i=0}^{n-1}\left(r_{i}^{\prime}(y, x)+s_{i}^{\prime}(y, x)\right) x^{i}(y-1)(x-1) .
\end{aligned}
$$

Agora, defina $\alpha:\left\langle x^{n}, y\right\rangle \rightarrow\langle x, y\rangle$ o homomorfismo que estende a função

$$
x^{n} \mapsto y, y \mapsto x .
$$

Pela Proposição 2.1.4, para que $f$ seja um homomorfismo bem definido, devemos $\operatorname{ter}\left(r(x, y) x^{n}\right)^{\mu}=r(x, y)^{\mu} y$ e $(r(x, y) y)^{\mu}=r(x, y)^{\mu} x$. De fato

$$
\begin{aligned}
& r(x, y) x^{n}=\sum_{i=0}^{n-1} r_{i}\left(x^{n}, 1\right) x^{n} x^{i}\left(x^{n}-1\right)+\sum_{i=0}^{n-1} s_{i}(1, y) x^{i}(y-1)+ \\
& +\sum_{i=0}^{n-1}\left(\left(r_{i}^{\prime}\left(x^{n}, y\right)+s_{i}^{\prime}\left(x^{n}, y\right)\right) x^{n}+s_{i}(1, y)\right) x^{i}\left(x^{n}-1\right)(y-1) .
\end{aligned}
$$

$e$

$$
\begin{gathered}
\left(r(x, y) x^{n}\right)^{\mu}=\sum_{i=0}^{n-1} r_{i}(y, 1) y x^{i}(y-1)+\sum_{i=0}^{n-1} s_{i}(1, x) x^{i}(x-1)+ \\
+\sum_{i=0}^{n-1}\left(\left(r_{i}^{\prime}(y, x)+s_{i}^{\prime}(y, x)\right) y+s_{i}(1, x)\right) x^{i}(y-1)(x-1)=r(x, y)^{\mu} y .
\end{gathered}
$$

Agora

$$
r(x, y) y=\sum_{i=0}^{n-1} r_{i}\left(x^{n}, 1\right) x^{i}\left(x^{n}-1\right)+\sum_{i=0}^{n-1} s_{i}(1, y) y x^{i}(y-1)+
$$




$$
+\sum_{i=0}^{n-1}\left(\left(r_{i}^{\prime}\left(x^{n}, y\right)+s_{i}^{\prime}\left(x^{n}, y\right)\right) y+r_{i}\left(x^{n}, 1\right)\right) x^{i}\left(x^{n}-1\right)(y-1) .
$$

$e$

$$
\begin{gathered}
(r(x, y) y)^{\mu}=\sum_{i=0}^{n-1} r_{i}(y, 1) x^{i}(y-1)+\sum_{i=0}^{n-1} s_{i}(1, x) x x^{i}(x-1)+ \\
+\sum_{i=0}^{n-1}\left(\left(r_{i}^{\prime}(y, x)+s_{i}^{\prime}(y, x)\right) x+r_{i}(y, 1)\right) x^{i}(y-1)(x-1)=r(x, y)^{\mu} x .
\end{gathered}
$$

\section{Como desejado.}

Vamos mostrar que $f$ é simples. Seja $\mathcal{J}$ um ideal de $\mathcal{A}$, contido em $\mathcal{I}$ e $\mu$ invariante. Suponha que $\mathcal{J}$ é não trivial. Podemos supor que os expoentes de $r$ em $x$ e y são positivos, pois $\mathcal{J}$ é um ideal. Seja $r(x, y) \in \mathcal{J} \backslash\{0\}$ tal que $\delta(r(x, y))$ é mínimo na ordem lexicográfica. Daí $r(x, y)^{\mu}=0$, pois $\delta\left(r^{\mu}\right)<\delta(r)$. Mas

$$
r=r_{0}+r_{1} x+\ldots+r_{n-j-1} x^{n-j-1}+r_{n-j} x^{n-j}+\ldots+r_{n-1} x^{n-1}
$$

com

$$
r_{i}=r_{i}^{(1)}\left(x^{n}\right)\left(x^{n}-1\right)+r_{i}^{(2)}(y)(y-1)+r_{i}^{(3)}\left(x^{n}, y\right)\left(x^{n}-1\right)(y-1) .
$$

Aplicando $\mu$

$$
r^{\mu}=r_{0}^{\mu}+r_{1}^{\mu} x+\ldots+r_{n-j-1}^{\mu} x^{n-j-1}+r_{n-j}^{\mu} x^{n-j}+\ldots+r_{n-1}^{\mu} x^{n-1}
$$

com

$$
r_{i}^{\mu}=r_{i}^{(1)}(y)(y-1)+r_{i}^{(2)}(x)(x-1)+r_{i}^{(3)}(y, x)(y-1)(x-1) .
$$

Assim

$$
\begin{aligned}
r & =r_{0}+r_{1} x+\ldots+r_{n-j-1} x^{n-j-1}+x^{n-j}\left(r_{n-j}+\ldots+r_{n-1} x^{j-1}\right), \\
r^{\mu} & =r_{0}^{\mu}+r_{1}^{\mu} x+\ldots+r_{n-j-1}^{\mu} x^{n-j-1}+x^{n-j}\left(r_{n-j}^{\mu}+\ldots+r_{n-1}^{\mu} x^{j-1}\right)
\end{aligned}
$$

$e$

$$
\begin{gathered}
x^{j} r=r_{0} x^{j}+r_{1} x^{j+1}+\ldots+r_{n-j-1} x^{n-1}+x^{n}\left(r_{n-j}+\ldots+r_{n-1} x^{j-1}\right) \\
\left(x^{j} r\right)^{\mu}=r_{0}^{\mu} x^{j}+r_{1}^{\mu} x^{j+1}+\ldots+r_{n-j-1}^{\mu} x^{n-1}+x\left(r_{n-j}^{\mu}+\ldots+r_{n-1}^{\mu} x^{j-1}\right) .
\end{gathered}
$$


Logo

$$
\left(x^{-j}\right)\left(x^{j} r\right)^{\mu}=r_{0}^{\mu}+r_{1}^{\mu} x+\ldots+r_{n-j-1}^{\mu} x^{n-j-1}+x^{1-j}\left(r_{n-j}^{\mu}+\ldots+r_{n-1}^{\mu} x^{j-1}\right)
$$

$e$

$$
r^{\mu}-x^{-j}\left(x^{j} r\right)^{\mu}=x^{1-j}\left(x^{n-1}-1\right)\left(r_{n-j}^{\mu}+\ldots+r_{n-1}^{\mu} x^{j-1}\right) .
$$

Assim

$$
\left(x^{n-1}-1\right)\left(r_{n-j}^{\mu}+\ldots+r_{n-1}^{\mu} x^{j-1}\right) \in \mathcal{J}
$$

e daí

$$
\left(x^{n-1}-1\right) r_{j}^{\mu} \in \mathcal{J}, \forall j=0, \ldots, n-1 .
$$

Para $j>0$ temos

$$
\delta\left(\left(x^{n-1}-1\right) r_{j}^{\mu}\right) \leqslant \delta\left(r_{j}\right)<\delta\left(r_{j} x^{j}\right)<\delta(r),
$$

daí $r_{j}=0$, para todo $j=1, \ldots, n-1$. Com isso

$$
r=r_{0}=r_{0}^{(1)}\left(x^{n}\right)\left(x^{n}-1\right)+r_{0}^{(2)}(y)(y-1)+r_{0}^{(3)}\left(x^{n}, y\right)\left(x^{n}-1\right)(y-1) .
$$

Como $r^{\mu}=0$ e r possui a forma acima, só podemos ter $r=0$, absurdo. Pela Proposição 2.2.1, $f$ é simples.

Podemos generalizar as ideias que aparecem nesse exemplo para mostrar que o grupo

$$
G_{n, d}=C_{n} \curlywedge C^{d}=\langle a\rangle \prec\left\langle x_{1}, \ldots, x_{d}\right\rangle,
$$

tem uma representação fechada por estado de grau $n^{n+d}$. Basta tomar

$$
H=\left\langle a^{x_{1}^{n}-1}, a^{x_{2}-1}, \ldots, a^{x_{d}-1}\right\rangle^{\left\langle x_{1}, \ldots, x_{d}\right\rangle}\left\langle x_{1}^{n}, x_{2}, \ldots, x_{d}\right\rangle,
$$

$\alpha: H \rightarrow G_{n, d}$ como o homomorfismo que estende a função

$$
x_{1}^{n} \mapsto x_{d}, x_{2} \mapsto x_{1}, \ldots, x_{d} \mapsto x_{d-1}
$$

e $\mu: \mathcal{I} \rightarrow \mathcal{A}$ o homomorfismo de grupos abelianos análogo ao do Exemplo 2.2.2, respeitando a escrita única de cada elemento de $\mathcal{I}$ e a definição de $\alpha$, que teremos um endomorfismo simples $f: H \rightarrow G$. Note que $[G: H]=n^{n+d}$. Assim podemos enunciar o seguinte teorema.

Teorema 2.2.3. $O$ grupo $G=C_{n}^{k} \curlywedge C^{d}$, possui uma representação fechada por estado de grau $n^{k(n+d)}$, para todos $d \geq 2$ e $k \geq 1$. 
Demonstração. Considere

$$
G=C_{n}^{k} \imath C^{d}=\left\langle a_{1}, \ldots, a_{k}\right\rangle \curlywedge\left\langle x_{1}, \ldots, x_{d}\right\rangle .
$$

Tome $H$ como o subgrupo

$$
\left\langle a_{i}^{x_{1}^{n}-1}, a_{i}^{x_{2}-1}, \ldots, a_{i}^{x_{d}-1} \mid i=1, \ldots, k\right\rangle^{\left\langle x_{1}, \ldots, x_{d}\right\rangle}\left\langle x_{1}^{n}, x_{2}, \ldots, x_{d}\right\rangle .
$$

Veja que $[G: H]=n^{k(n+d)}$. Defina $f: H \rightarrow G$ análoga à observação acima.

No Capítulo 3, veremos que o grupo $G_{B, 1}=B$ ح $C$ possui uma representação fechada por estado de grau $|B|$. Já no Teorema 2.2.3, a representação de $C_{n}^{k}$ ح $C^{d}$ é de grau $\left|C_{n}^{k}\right|^{n+d}$. Naturalmente, nos perguntamos se é possível ter uma representação com grau $\left|C_{n}^{k}\right|$, ou seja, um autômato sobre um alfabeto com $\left|C_{n}^{k}\right|$ letras. Na seção seguinte, supondo que $n$ é primo e $k=1$, demonstramos que isso não ocorre.

\subsection{Não existência de endomorfismo simples de grau primo para $G_{p, d}, d \geq 2$}

Nesta seção demonstramos que não existe endomorfismo simples de grau primo para $G_{p, d}$, onde $p$ é primo e $d$ é maior ou igual a 2 .

Proposição 2.3.1. Sejam $M=\left(m_{i j}\right)_{d \times d}$ uma matriz quadrada de ordem $d \geq 2, r$ um inteiro não nulo, $t=\operatorname{det}(M) e \mathbb{K}$ um corpo. Se $u_{1}, \ldots, u_{d} \in \mathbb{K}\left\langle x_{1}, \ldots, x_{d}\right\rangle$ são tais que

$$
\left(x_{1}^{r m_{i 1}} \ldots x_{d}^{r m_{i d}}-1\right) u_{j}=\left(x_{1}^{r m_{j 1}} \ldots x_{d}^{r m_{j d}}-1\right) u_{i},
$$

para todos $1 \leq i<j \leq d$, então $t=0$ ou $u_{i} \in \mathcal{I}=\left\langle x_{1}^{r}-1, \ldots, x_{d}^{r}-1\right\rangle_{\text {ideal }}$, para todo $i=1, \ldots, d$.

Demonstração. Vamos aplicar indução sobre $d \geq 2$. Seja então $d=2$. Suponha que $p=p\left(x_{1}, x_{2}\right) \in \mathbb{K}\left\langle x_{1}, x_{2}\right\rangle$ é um fator comum não constante de $x_{1}^{r m_{11}} x_{2}^{r m_{12}}-1$ e $x_{1}^{r m_{21}} x_{2}^{r m_{22}}-1$. Módulo $p$ temos

$$
\begin{gathered}
\left(x_{1}^{r m_{11}} x_{2}^{r m_{12}}\right)^{m_{22}} \cdot\left(x_{1}^{r m_{21}} x_{2}^{r m_{22}}\right)^{-m_{12}}=1=x_{1}^{r m_{11} m_{22}-r m_{21} m_{22}} x_{2}^{r m_{12} m_{22}-r m_{12} r m_{22}}= \\
=x_{1}^{r m_{11} m_{22}-r m_{21} m_{22}}=x_{1}^{r t},
\end{gathered}
$$


isto é, $p \mid\left(x_{1}^{r t}-1\right)$. Analogamente, $p \mid\left(x_{2}^{r t}-1\right)$. Logo, existem $q=q\left(x_{1}, x_{2}\right)$ e $q^{\prime}=q^{\prime}\left(x_{1}, x_{2}\right)$ em $\mathbb{K}\left\langle x_{1}, x_{2}\right\rangle$ tais que

$$
x_{1}^{r t}-1=p q, x_{2}^{r t}-1=p q^{\prime} .
$$

Desde que $\mathbb{K}\left\langle x_{1}, x_{2}\right\rangle$ é domínio de fatoração única, então $p, q \in \mathbb{K}\left\langle x_{1}\right\rangle$ e $p, q \in$ $\mathbb{K}\left\langle x_{2}\right\rangle$. Portanto, $p \in \mathbb{K}$, uma contradição. Concluímos que

$$
\left(x_{1}^{r m_{21}} x_{2}^{r m_{22}}-1\right)\left|u_{1},\left(x_{1}^{r m_{11}} x_{2}^{r m_{12}}-1\right)\right| u_{2}
$$

e $u_{1}, u_{2} \in \mathcal{I}=\left\langle x_{1}^{r}-1, x_{2}^{r}-1\right\rangle_{\text {ideal }}$.

Suponha agora que $d \geq 3$ e que a afirmação é verdadeira para $d-1$. Suponha, por absurdo, que $\operatorname{det}(M) \neq 0 \mathrm{e}$, sem perda de generalidade, que $u_{1}$ não é um elemento de $\mathcal{I}$. Denote por $M_{i j}$ a matriz obtida de $M$ suprimindo sua linha $i$ e sua coluna $j$. Fazendo $x_{i}=1(1 \leq i \leq d)$ em

$$
\left(x_{1}^{r m_{11}} x_{2}^{r m_{12}} \ldots x_{d}^{r m_{1 d}}-1\right) u_{i}=\left(x_{1}^{r m_{i 1}} x_{2}^{r m_{i 2}} \ldots x_{d}^{r m_{i d}}-1\right) u_{1}
$$

com $i \neq 1$, vamos obter, por hipótese de indução, $\operatorname{det}\left(M_{i j}\right)=0$ para todo $(i, j)$, onde $i \neq 1$. Assim

$$
\operatorname{det}(M)=\sum_{i=1}^{d}(-1)^{i+j} m_{i j} \operatorname{det}\left(M_{i j}\right)=(-1)^{1+j} m_{1 j} \operatorname{det}\left(M_{1 j}\right),
$$

para todo $j=1, \ldots, d$. Por outro lado, $\operatorname{det}(M)=\sum_{j=1}^{d}(-1)^{1+j} m_{i j} \operatorname{det}\left(M_{1 j}\right)$ e daí

$$
\sum_{j=1}^{d} \operatorname{det}(M)=\sum_{j=1}^{d}(-1)^{1+j} m_{i j} \operatorname{det}\left(M_{1 j}\right)=\operatorname{det}(M),
$$

ou seja

$$
(d-1) \operatorname{det}(M)=0 \Rightarrow \operatorname{det}(M)=0 .
$$

Uma contradição. A proposição segue.

Suponha que $H$ é um subgrupo de índice $q$ em $G_{p, d}$, com $q$ primo, e $f: H \rightarrow$ $G$ é um endomorfismo simples. Assim $\left[G_{p, d}: A H\right]=1$ ou $q$. Se $\left[G_{p, d}: A H\right]=q$, então $[A H: H]=1$ e $A$ é um subgrupo de $H$, o que não ocorre pois $f$ é simples. Daí $\left[G_{p, d}: A H\right]=1$ e $Y=A H \cap X=X$. Como $A$ é abeliano, segue que $A_{0}=H \cap A$ é normal em $G_{p, d}$. Pelo Corolário 2.1.7, podemos supor $H=A_{0} X$. Usando a notação da Proposição 2.2.1, vamos provar que existe um ideal não trivial $\mathcal{J}$ de $\mathcal{A}$ e $\mu$-invariante. 
Proposição 2.3.2. Seguindo as notações da Proposição 2.2.1, com $n=p e\left[G_{p, d}\right.$ : $H]=q$, com q primo, existe um ideal não trivial $\mathcal{J}$ de $\mathcal{A}$ e $\mu$-invariante.

Demonstração. Note que $\mathcal{A} / \mathcal{I}$ é finito, então se $r$ é o expoente do grupo das unidades do anel quociente $\mathcal{A} / \mathcal{I}$, segue que o ideal $\mathcal{I}$ contém o ideal

$$
\mathcal{J}=\left\langle x_{1}^{r}-1, \ldots, x_{d}^{r}-1\right\rangle_{\text {ideal }} .
$$

Pela Proposição 2.2.1, $\alpha: X \rightarrow X$ é monomorfismo, logo existe uma matriz $M=\left(m_{i j}\right)_{d \times d}, \operatorname{com} \operatorname{det}(M) \neq 0$, tal que

$$
x_{i}^{\alpha}=x_{1}^{r m_{i 1}} \ldots x_{d}^{r m_{i d}}
$$

para todo $i=1, \ldots d$. Pondo $\left(x_{i}^{r}-1\right)^{\mu}=u_{i}$ e tomando $i \neq j$, temos por um lado

$$
\left(\left(x_{i}^{r}-1\right)\left(x_{j}^{r}-1\right)\right)^{\mu}=\left(x_{i}^{r}-1\right)^{\mu}\left(x_{j}^{r}-1\right)^{\alpha}=\left(x_{1}^{r m_{j 1}} \ldots x_{d}^{r m_{j d}}\right) u_{i}
$$

e por outro

$$
\left(\left(x_{j}^{r}-1\right)\left(x_{i}^{r}-1\right)\right)^{\mu}=\left(x_{j}^{r}-1\right)^{\mu}\left(x_{i}^{r}-1\right)^{\alpha}=\left(x_{1}^{r m_{i 1}} \ldots x_{d}^{r m_{i d}}\right) u_{j},
$$

ou seja, $\left(x_{1}^{r m_{i 1}} \ldots x_{d}^{r m_{i d}}-1\right) u_{j}=\left(x_{1}^{m_{j 1}} \ldots x_{d}^{m_{j d}}-1\right) u_{i}$, para todos $1 \leq i<j \leq d$. Pela Proposição 2.3.1, temos que $u_{i} \in \mathcal{J}$ para todo $i=1, \ldots, d$. Portanto, $\mathcal{J}$ é um ideal de $\mathcal{A}$, contido em $\mathcal{I}$ e $\mu$-invariante.

Por essa proposição e pela Proposição 2.2.1, temos o teorema:

Teorema 2.3.3. Não existe endomorfismo simples $f: H \rightarrow G_{p, d}$ tal que o índice $\left[G_{p, d}: H\right]$ é primo, onde pé primo. 
CAPÍTULO 3

\section{Representações fechadas por estado de grupos Lamplighter generalizados}

Neste capítulo passaremos a classificar agumas representações fechadas por estado de grupos da forma $G_{B, 1}=B \nmid C$, com $B$ grupo abeliano finito. Silva e Steinberg chamam tais grupos de grupos Lamplighter generalizados. Também demonstraremos que um grupo abeliano livre de rank infinito não possui uma representação transitiva fechada por estado de grau $p$, com $p$ primo. Como consequência, o grupo $C$ ? $C$ também não possui tal representação.

\subsection{Representações de grupos Lamplighter genera- lizados}

Aqui analisaremos dois autômatos que definem $G_{B, 1}$, para logo em seguida enunciar um resultado geral sobre representações que induzem ou são induzidas por autômatos de tais tipos.

\section{Autômato de Silva e Steinberg}

Dado um autômato $A=(Q, \Gamma, f, l)$, uma letra $a \in \Gamma$ é dita ser resetada se para quaisquer $\alpha, \beta \in Q$ tem-se que $\left.\alpha\right|_{a}=\left.\beta\right|_{a}$, onde $\left.\alpha\right|_{a}=(\alpha, a) l$ e $\left.\beta\right|_{a}=(\beta, a) l$. Ou seja, a letra $a$ reseta o autômato $A$ para o estado $\eta=\left.\alpha\right|_{a}$. Se toda letra de um 
autômato é resetada, chamaremos tal autômato de autômato resetado.

Agora, considere $G$ um grupo finito. Defina a máquina de Cayley $\mathcal{C}(M)$, em relação a $G$, como o autômato $\mathcal{C}(G)=(G, G, *, *)$, onde * é a operação de $G$, ou seja, o alfabeto e o conjunto de estados são dados por $G$ e as funcões de mudança e de saída são dadas pela operação de $G$. Como exemplo, $\mathcal{C}\left(C_{3}\right)$ é dado por

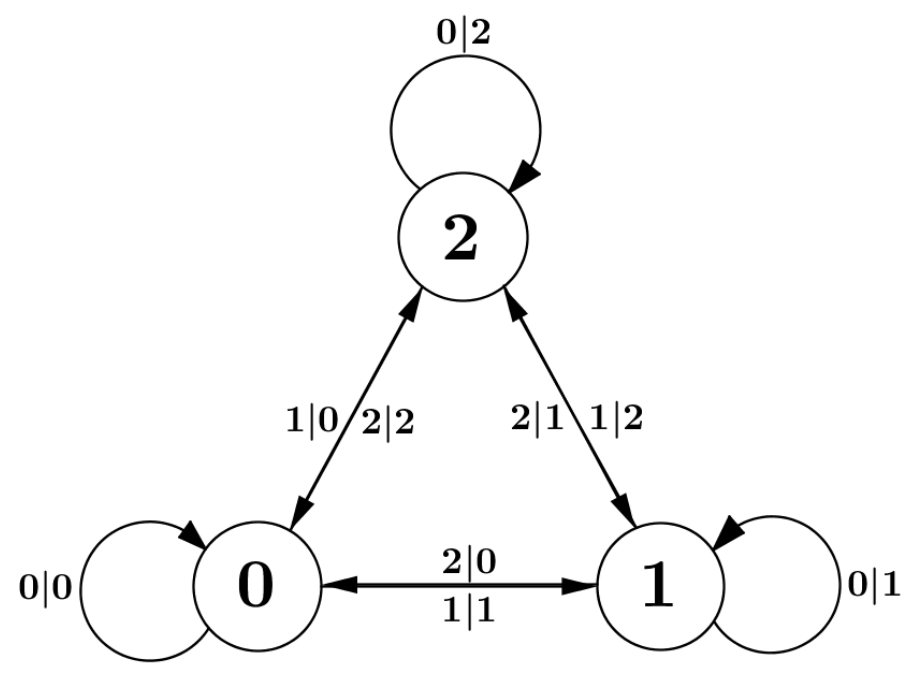

Figura 3.1: Diagrama de $\mathcal{C}\left(C_{3}\right)$.

Note que o grupo $\mathcal{G}\left(\mathcal{C}\left(C_{3}\right)\right)$ gerado por $\mathcal{C}\left(C_{3}\right)$ é dado por

$$
\mathcal{G}\left(\mathcal{C}\left(C_{3}\right)\right)=\langle\alpha=(\alpha, \beta, \gamma), \beta=(\beta, \gamma, \alpha)(012), \gamma=(\gamma, \alpha, \beta)(021)\rangle .
$$

Vale que $\mathcal{G}\left(\mathcal{C}\left(C_{3}\right)\right) \simeq \mathcal{G}\left(\mathcal{C}\left(C_{3}\right)^{-1}\right) \simeq C_{3} \curlyvee C$. De maneira mais geral, Silva e Steinberg demonstraram que:

Teorema 3.1.1. ([24]) Se B é um grupo abeliano finito não trivial, então $\mathcal{C}(B)^{-1}$ é um autômato resetado e $\mathcal{G}\left(\mathcal{C}(B)^{-1}\right) \simeq \mathcal{G}(\mathcal{C}(B)) \simeq B$ 々 $C$.

Dando assim uma representação fechada por estado de grau $|B|$ para $B$ 々 $C$. Pela definição da Máquina de Cayley, é fácil ver que

$$
\mathcal{G}(\mathcal{C}(B))=\left\langle\alpha_{0}, \ldots, \alpha_{|B|-1}\right\rangle
$$

onde

$$
\alpha_{i}=\left(\alpha_{(0)^{\sigma\left(\alpha_{i}\right)}}, \ldots, \alpha_{(|B|-1)^{\sigma\left(\alpha_{i}\right)}}\right) \sigma\left(\alpha_{i}\right)
$$


e $\sigma\left(\alpha_{i}\right)$ é a permuatação induzida em $B$ por $b_{i}, \operatorname{com}\left\{b_{0}, \ldots, b_{|B|-1}\right\}$ uma enumeração de $B$. Podemos supor que $b_{0}=1$, assim

$$
\alpha_{0}=\left(\alpha_{0}, \alpha_{1}, \ldots, \alpha_{|B|-1}\right) .
$$

Como $\mathcal{C}(B)^{-1}$ é resetado, segue que

$$
\mathcal{G}\left(\mathcal{C}(B)^{-1}\right)=\left\langle\alpha_{0}, \alpha_{0} \sigma\left(\alpha_{1}\right)^{-1}, \ldots, \alpha_{0} \sigma\left(\alpha_{|B|-1}\right)^{-1}\right\rangle .
$$

Daí

$\mathcal{G}\left(\mathcal{C}(B)^{-1}\right)=\left\langle\alpha_{0}, \sigma\left(\alpha_{1}\right)^{-1}, \ldots, \sigma\left(\alpha_{|B|-1}\right)^{-1}\right\rangle=\left\langle\sigma\left(\alpha_{1}\right)^{-1}, \ldots, \sigma\left(\alpha_{|B|-1}\right)^{-1}\right\rangle \chi\left\langle\alpha_{0}\right\rangle$,

onde $\left\langle\sigma\left(\alpha_{1}\right)^{-1}, \ldots, \sigma\left(\alpha_{|B|-1}\right)^{-1}\right\rangle \simeq B$ e $\left\langle\alpha_{0}\right\rangle \simeq C$. Por fim, note que

$$
\left\langle\alpha_{0}\right\rangle \leqslant \operatorname{Fix}_{\mathcal{G}\left(\mathcal{C}(B)^{-1}\right)}(0)=\operatorname{Stab}_{\mathcal{G}\left(\mathcal{C}(B)^{-1}\right)}(1) .
$$

Para mais detalhes consulte [14] e [24].

\section{Autômato de Bartholdi e Sunik}

Parecido com o autômato anterior, esse autômato também é definido sobre grupos da forma $G_{B, 1}=B \imath C$, mas $B=C_{n}^{k}$, para $n \geq 2 \mathrm{e} k$ inteiro positivo. Considere o alfabeto $\Gamma=\mathbb{Z} / n \mathbb{Z}$, o conjunto de estados $Q=(\mathbb{Z} / n \mathbb{Z})^{k}$ e um polinômio mônico $p(t)=a_{0}+a_{1} t+\ldots a_{k} t^{k}$ de grau $k$ que é invertível sobre o anel $(\mathbb{Z} / n \mathbb{Z})[[t]]$. Assim podemos considerar $a_{0}$ invertível em $\mathbb{Z} / n \mathbb{Z}$ e $a_{k}=1$. Defina a função $g: \Gamma^{k+1} \rightarrow \Gamma$ por $\left(x_{0}, x_{1}, \ldots, x_{k}\right) g=a_{k} x_{0}+a_{k-1} x_{1}+\ldots+a_{0} x_{k}$. Agora defina o autômato $A_{p}=(Q, \Gamma, f, l)$, onde

$$
\left(\left(x_{1}, \ldots, x_{k}\right), x\right) f=\left(\left(x_{1}, \ldots, x_{k}\right), x\right) g \text { e }\left(\left(x_{1}, \ldots, x_{k}\right), x\right) l=\left(x_{2}, \ldots, x_{k}, x\right)
$$

para todo $x \in \Gamma$ e todo $\left(x_{1}, \ldots, x_{k}\right) \in Q$.

Como exemplo, as funções $f$ e $l$ do autômato $A_{1+t}$, onde $n=3$ e $k=1$, são dadas por $(x, y) f=x+y$ e $(x, y) l=y$, para todos $x, y \in \Gamma=Q=\mathbb{Z} / n \mathbb{Z}$. Em particular, esse autômato é equivalente ao autômato da Figura 3.1.

Em [5], Bartholdi e Sunik, provam que:

Teorema 3.1.2. ([5, página 8]) O autômato $A_{p}$ é tal que $\mathcal{G}\left(A_{p}\right)$ é isomorfo a $G_{B, 1}$, onde $B=C_{n}^{k}$.

Para tanto, eles demonstram que $\mathcal{G}\left(A_{p}\right)$ é o grupo de automorfismos

$$
\left\langle\sigma, \sigma^{(1)}, \ldots, \sigma^{(k-1)}, \alpha\right\rangle
$$


de $\mathcal{T}_{n}$, onde $\sigma=(01 \ldots n-1)$ e $\alpha$ é o automorfismo obtido quando se faz corresponder cada elemento $u=a_{0} a_{1} a_{2} \ldots a_{r}$ de $\mathcal{T}_{m}$ a um polinômio

$$
p_{u}(t)=a_{0}+a_{1} t+a_{2} t^{2}+\ldots+a_{r} t^{r} \text { sobre } \mathbb{Z} / n \mathbb{Z}
$$

e define

$$
u^{\alpha}=\left[p(t) p_{u}(t)\right],
$$

onde $\left[p(t) p_{u}(t)\right]$ é o polinômio de grau no máximo $r$ formado pelos $r+1$ primeiros termos do polinômio $p(t) p_{u}(t)$. Por exemplo, se $p(t)=p_{u}(t)=1+t$, então $\left[p(t) p_{u}(t)\right]=1+2 t$. Note que no grupo de automorfismos $\left\langle\sigma, \sigma^{(1)}, \ldots, \sigma^{(k-1)}, \alpha\right\rangle$, $0^{\alpha}=0$ e a ordem de $\alpha$ é infinita, assim $\alpha \in F i x_{\mathcal{G}\left(A_{g}\right)}(0)$. Para mais detalhes consulte [3].

Observe também, que esses dois autômatos nem sempre são equivalentes, pois $\operatorname{Fix}_{\mathcal{G}\left(A_{g}\right)}(0)$ nem sempre é normal em $\mathcal{G}\left(A_{g}\right)$, enquanto que $\operatorname{Fix}_{\mathcal{G}\left(\mathcal{C}(B)^{-1}\right)}(0)$ é normal em $\mathcal{C}(B)^{-1}$. Mais ainda, o primeiro autômato tem grau $|B|$, enquanto que o segundo tem grau $n$.

Mas temos uma semelhança entre esses dois eles, qual seja, o endomorfismo $\varphi:$ Fix $_{G}(0) \rightarrow G$ é sobrejetor em cada um deles, logo, recorrente. Pela Proposição 2.1.8, segue que podemos garantir que essas representações induzem representações do grupo $\oplus_{C} B$ (ou $\oplus_{C} C_{n}^{k}$ ) de grau $|B|$ (ou $n$ ). De maneira mais geral, temos:

Proposição 3.1.3. Se $f: H \rightarrow G_{B, 1}$ é um endomorfismo simples de grau n, onde $H$ se projeta sobrejetivamente sobre $C$, então $f: A_{0} \rightarrow A$ é simples, com $A_{0}=H \cap A$ e $A=\oplus_{C} B$.

Logo, de um endomorfismo simples de produto entrelaçado passamos para um endomorfismo simples de um grupo abeliano. Pelo Teorema 1.1.13, o expoente de $B$ e $n$ estão ligados. Quando $n=p$ é primo, o expoente de $B$ só pode ser $p$, ou seja, $B$ deve ser um grupo $p$-abeliano elementar. Na próxima seção analisaremos representações fechadas por estado de grupos do caso mais "simples", quando $B=C_{p}$

\subsection{Representações do grupo Lamplighter $C_{p} \prec C$}

Demonstraremos aqui, que uma representação fechada por estado de um grupo Lamplighter $G_{p, 1}=C_{p} \succ C$ é equivalente a apenas dois tipos de representações. Tais tipos estão explícitos nos Teoremas 3.2.1 e 3.2.3. 
Teorema 3.2.1. Suponha que $H$ é um subgrupo normal de $G_{p, 1}$ de índice $p$. Então cada representação fechada por estado de $G_{p, 1}$ sobre a árvore uniraiz p-regular com respeito a $H$ é reduzida a $\varphi: G_{p, 1} \rightarrow \mathcal{A}_{p}$, onde

$$
\begin{gathered}
a \mapsto a^{\varphi}=\sigma=(01 \ldots p-1) \\
x \mapsto x^{\varphi}=\xi=\left(\xi^{n}, \xi^{n} \sigma^{u(\xi)}, \ldots, \xi^{n} \sigma^{u(\xi)(p-1)}\right)
\end{gathered}
$$

para algum inteiro $n$ e algum polinômio de Laurent $u(x) \in \mathbb{K}\langle x\rangle$, tais que $m d c(p, n)=1$ e $u(1) \neq 0$.

Demonstração. Seja $f: H \rightarrow G_{p, 1}$ um endomorfismos simples. Pela Proposição 2.1.5, podemos substituir $H$ por $A_{0}\langle x\rangle=G^{\prime}\langle x\rangle$ e $f$ por um par de homomorfismos $(\mu, \alpha)$, tais que $\mu: \mathcal{I} \rightarrow \mathcal{A}$, $\operatorname{com} \mathcal{I} \simeq A_{0}$ e $\mathcal{A} \simeq A$, e $\alpha: X \rightarrow X$ é monomorfismo, com $X \simeq C \simeq\langle x\rangle$. Seja $n$ tal que $x^{\alpha}=x^{n}$. Como $\mathcal{I}$ corresponde ao ideal $\langle x-1\rangle$ de $\mathcal{A}=K\langle x\rangle$, segue que

$$
\mu: p(x)(x-1) \mapsto p(x)^{\alpha}(x-1)^{\mu}=p\left(x^{n}\right) u(x),
$$

para todo $p(x) \in \mathcal{A}$. Como $f$ é simples, pela Proposição 3.1.3, segue que $\mu$ é simples, logo $m d c(u(x), x-1)=1$, ou seja, $u(1) \neq 0$.

Agora suponha que $n=p n^{\prime}$. Assim

$$
\begin{gathered}
\left(p(x)(x-1)^{2}\right)^{\mu}=(p(x)(x-1))^{\alpha}(x-1)^{\mu}=p\left(x^{n}\right)\left(x^{n}-1\right) u(x)= \\
=p\left(x^{n}\right)\left(x^{n^{\prime}}-1\right)^{p} u(x)=t(x)(x-1)^{2},
\end{gathered}
$$

ou seja, o ideal $\mathcal{J}=\mathcal{I}(x-1)^{2}$ é $\mu$-invariante, absurdo. $\operatorname{Logo} m d c(p, n)=1$.

Podemos tomar $T=\left\{1, a, a^{2}, \ldots, a^{p-1}\right\}$ como um transversal de $H$ em $G$. Note que $x$ induz a permutação trivial em $T$, pois $x \in H$ e $H$ é normal. Com relação a esse transversal e a $f$, obtemos a seguinte representação de $G_{p, 1}$ :

$$
a^{\varphi}=\left(\left[a^{i} a a^{-i-1}\right]^{f \varphi}\right)_{i=0, \ldots, p-1}(01 \ldots p-1)=(01 \ldots p-1)=\sigma
$$

$\mathrm{e}$

$$
\begin{aligned}
x^{\varphi}=\left(\left[a^{i} x a^{-i}\right]^{f \varphi}\right)_{i=0, \ldots, p-1} & =\left(\left[x a^{i x-i}\right]^{f \varphi}\right)_{i=0, \ldots, p-1}=\left(\left[x a^{(x-1) i}\right]^{f \varphi}\right)_{i=0, \ldots, p-1}= \\
& =\left(x^{\varphi} a^{\varphi u\left(x^{\varphi}\right) i}\right)_{i=0, \ldots, p-1},
\end{aligned}
$$

ou seja, $x^{\varphi}=\xi=\left(\xi^{n}, \xi^{n} \sigma^{u(\xi)}, \ldots, \xi^{n} \sigma^{\mu(\xi)(p-1)}\right)$. 
Agora, vamos mostrar que se escolhermos $n$ e $u(x)$ tais que $m d c(p, n)=1 \mathrm{e}$ $u(1) \neq 0$, então $f$ que é induzida por $\alpha: X \rightarrow X$ e $\mu: \mathcal{I} \rightarrow \mathcal{A}$, tais que

$$
x^{\alpha}=x^{n},(p(x)(x-1))^{\mu}=p\left(x^{n}\right) u(x), \forall p(x) \in \mathcal{A},
$$

é simples. De fato, se $\Phi_{n}(x)=\left(x^{n}-1\right) /(x-1)$, então $\Phi_{n}(1) \neq 1$, pois $m d c(p, n)=1$. Agora se $p(x)(x-1)^{j}$ é um polinômio não nulo de $\mathcal{I}$, com $j \geqslant 1$ e $p(1) \neq 0$, então

$$
\begin{aligned}
\left(p(x)(x-1)^{j}\right)^{\mu}= & \left((x-1)^{j-1} p(x)\right)^{\alpha}(x-1)^{\mu}=\left(x^{n}-1\right)^{j-1} p\left(x^{n}\right) u(x)= \\
& =(x-1)^{j-1}\left(\Phi_{n}(x)^{j-1} p\left(x^{n}\right) u(x)\right) .
\end{aligned}
$$

Como $\Phi_{n}(1)^{j-1} p(1) u(1) \neq 0$, segue que $\left(p(x)(x-1)^{j}\right)^{\mu^{j}}$ não está em $\mathcal{I}$. Portanto, $\mu$ é simples e, assim, $f$ é simples. Podemos então enunciar:

Proposição 3.2.2. Se $m d c(p, n)=1$ e $u(x) \in K\langle x\rangle$ é tal que $u(1) \neq 0$, então o subgrupo

$$
\left\langle\sigma=(01 \ldots p-1), \xi=\left(\xi^{n}, \xi^{n} \sigma^{u(\xi)}, \ldots, \xi^{n} \sigma^{u(\xi)(p-1)}\right)\right\rangle
$$

de $\mathcal{A}_{p}$ é um subgrupo transitivo fechado por estado e isomorfo a $G_{p, 1}$.

No caso em que $H$ não é normal em $G_{p, 1}$ e $\left[G_{p, 1}: H\right]=p$, segue que $G=$ $A H, \log A H \cap X=X$, ou seja, $H$ se projeta sobrejetivamente sobre $X$. Daí $A_{0}=A \cap H$ é normal em $G$ e ainda podemos usar a Proposição 2.1.5. Assim podemos considerar $T=\left\{1, a, \ldots, a^{p-1}\right\}$ um transversal de $H$ em $G_{p, 1}$. Como $H$ não é normal, $x$ induz uma permutação $\tau$ não trivial em $T$.

Afirmamos que $\tau$ é definida por $\tau: i \mapsto i c$, para todo $i \in\{0,1, \ldots, p-1\}$, com $c \in\{1, \ldots, p\}$ fixo. De fato, temos que $a^{i} x a^{-j}=x a^{i x-j} \in H$, onde $i^{\tau}=j$. Mas $a^{i x-j}$ está em $A, \operatorname{logo}$ em $A_{0}$. Olhando $A_{0} \simeq \mathcal{I}$ e $A \simeq \mathcal{A}=K\langle x\rangle$, como $\mathcal{A} / \mathcal{I}=K$, segue que $\mathcal{I}=\langle x-c\rangle$ e $j=i c$. Como desejado.

Aplicando argumento análogo ao do Teorema 3.2.1 e Proposição 3.2.2, obtemos a seguir o teorema mais geral:

Teorema 3.2.3. Suponha que $H$ é um subgrupo de $G_{p, 1}$ de índice $p$. Então cada representação fechada por estado de $G_{p, 1}$ sobre a árvore uniraiz p-regular com respeito a $H$ é reduzida a $\varphi: G_{p, 1} \rightarrow \mathcal{A}_{p}$, onde

$$
\begin{gathered}
a \mapsto a^{\varphi}=\sigma=(01 \ldots p-1) \\
x \mapsto x^{\varphi}=\xi=\left(\xi^{n}, \xi^{n} \sigma^{u(\xi)}, \ldots, \xi^{n} \sigma^{u(\xi)(p-1)}\right) \tau
\end{gathered}
$$

onde $\tau: i \mapsto i c(\bmod p)$, com $c \in\{1, \ldots, p\}$ e n e o polinômio de Laurent $u(x) \in$ $K\langle x\rangle$ são tais que $\operatorname{mdc}(p, n)=1$ e $u(c) \neq 0$. 
3.3 Não existência de representação de grau primo para um grupo abeliano livre de rank infinito

Se $u(x)=1$ e $n=1$, então a representação $\varphi: G_{p, 1} \rightarrow \mathcal{A}_{p}$ é dada por

$$
\begin{gathered}
a \mapsto a^{\varphi}=\sigma=(01 \ldots p-1) \\
x \mapsto x^{\varphi}=\xi=\left(\xi, \xi \sigma, \ldots, \xi \sigma^{(p-1)}\right) \tau .
\end{gathered}
$$

Note que essa representação é finita por estado; de fato, $G^{\varphi}$ pode ser gerado pelos $p$ estados de $\xi$. Para $p=2$ temos a representação clássica do grupo Lamplighter $G_{2,1}=C_{2} \succ C$, dada por $a \mapsto \sigma=(01)$ e $x \mapsto \xi=(\xi, \xi \sigma)$. Agora, se $p=$ 3 , então $a \mapsto \sigma=(012)$ e $x \mapsto \xi=\left(\xi, \xi \sigma, \xi \sigma^{2}\right)(12)$, que é equivalente à representação induzida pelo autômato de [6]. Agora, o autômato de $L_{p, 1}=C_{p} \curlywedge C$, definido em [5] e discutido na seção anterior, induz uma representação equivalente à representação de $G_{p, 1}$ dada no Teorema 3.2.3, onde os autores definem $L_{n, d}=$ $C_{n}^{d} \prec C$.

\subsection{Não existência de representação de grau primo para um grupo abeliano livre de rank infinito}

Como o título diz, nesta seção demonstraremos que não existe uma representação transitiva fechada por estado de grau primo para um grupo abeliano livre de rank infinito.

Considere $\alpha=\left(e, \ldots, e, \alpha^{t^{i-1}}\right) \sigma \in \mathcal{A}_{p}$, onde $p$ é primo, $i$ é um inteiro positivo, $\alpha^{t^{k}}=\alpha^{(k)}$ é a $m$-upla $\left(\alpha^{k-1}, \ldots, \alpha^{k-1}\right)$ e $\sigma=(01 \ldots p-1)$. Os estados de $\alpha$ são $\alpha, \alpha^{t}, \ldots, \alpha^{t^{i-1}}$. Defina $D_{p}(i)$ como o subgrupo de $\mathcal{A}_{p}$ gerado pelos elementos $\alpha, \alpha^{t}, \ldots, \alpha^{t^{i-1}}$. É fácil ver que $D_{p}(i)$ é um grupo abeliano transitivo, fechado por estado e diagonalmente fechado.

Proposição 3.3.1. Se $\beta \in \overline{D_{p}(i)}$, então $\beta$ é um estado de $\beta^{n}$ para todo $n$ inteiro positivo.

Demonstração. Basta mostra para $\alpha$, isto é, $\alpha$ é um estado de $\alpha^{n}$, para todo $n$ inteiro positivo. Se $n=1$, o resultado é óbvio. Como $\alpha^{n+1}=\alpha^{n} \alpha$ e os estados de $\alpha^{n} \alpha$ são potências $\alpha^{r}$, onde $r<n+1$, segue, por hipótese de indução, que $\alpha$ é um estado de $\alpha^{n+1}$.

Considere $A$ um subgrupo abeliano livre de torção transitivo e fechado por estado de $\mathcal{A}_{p}$. Seja $i$ o menor dos inteiros positivos $j$ 's tais que

$$
\beta^{p} \in \operatorname{Stab}_{A}(j) \backslash \operatorname{Stab}_{A}(j+1),
$$


3.3 Não existência de representação de grau primo para um grupo abeliano livre de rank infinito

para $\beta$ variando em $A \backslash \operatorname{Stab}_{A}(1)$. O próximo resultado será usado e pode ser encontrado em [3, Teorema 9].

Teorema 3.3.2. (Brunner e Sidki) Sejam A um subgrupo abeliano livre de torção transitivo e fechado por estado de $\mathcal{A}_{p}$ e $\beta \in A \backslash \operatorname{Stab}_{A}(1)$, tal que $\beta^{p} \in \operatorname{Stab}_{A}(i) \backslash$ $\operatorname{Stab}_{A}(i+1)$, onde i é como acima. Então $A^{*}=\langle\beta\rangle^{*} e$, assim, $A^{*}$ é conjugado para $\overline{D_{p}(i)}$.

Vamos mostrar que um grupo abeliano livre fechado por estado e transitivo possui rank finito.

Teorema 3.3.3. Todo subgrupo abeliano livre transitivo fechado por estado de $\overline{D_{p}(i)}$ possui rank menor ou igual a $i$.

Demonstração. Suponha por absurdo, que exista um subgrupo $A$ abeliano livre transitivo fechado por estado de $\overline{D_{p}(i)}$ com rank maior que $i$. Como $\overline{D_{p}(i)}$ é um $\mathbb{Z}_{p}$-módulo topologicamente gerado por $\alpha, \alpha^{t}, \ldots, \alpha^{t^{i-1}}$, podemos encontrar um conjunto

$$
\left\{\alpha^{q_{1}(t)}, \alpha^{q_{2}(t)}, \ldots, \alpha^{q_{n}(t)}, \ldots\right\}
$$

de geradores livres de $A$, onde $n>i$,

$$
q_{j}(t) \in\left\{p(t)=a_{0}+a_{1} t+\ldots+a_{i-1} t^{i-1} ; a_{r} \in \mathbb{Z}_{p}, r=0, \ldots, i-1\right\}
$$

para todo $j=1,2, \ldots$, o primeiro nível da ação não trivial desses elementos é estritamente decrescente e $\alpha^{q_{1}}$ age não trivialmente no nível 1 da árvore $\mathcal{T}_{p}$. Tome o menor inteiro positivo $s$ tal que $\alpha^{q_{s}(t)}$ está em $\operatorname{Stab}_{A}(i)$. Como $\alpha^{q_{s}(t)} \in \overline{D_{p}(i)}$ e $\alpha^{q_{s}(t)}$ não age no nível $i$, existem $b_{1}(t), \ldots, b_{i}(t) \in \mathbb{Z}_{p}[t]$ tais que

$$
\alpha^{q_{s}(t)}=\alpha^{p b_{1}(t)} \alpha^{p t b_{2}(t)} \ldots \alpha^{p t^{i-1} b_{i}(t)}=\beta^{p},
$$

onde $\beta=\alpha^{b_{1}(t)} \alpha^{t b_{2}(t)} \ldots \alpha^{t^{i-1}} b_{1}(t)$. Pela Proposição 3.3.1, $\beta$ é um estado de $\beta^{p}$, $\operatorname{logo} \beta \in A$. Daí existem inteiros $l_{1}, \ldots, l_{k}$ tais que

$$
\beta=\alpha^{l_{1} q_{1}(t)} \alpha^{l_{2} q_{2}(t)} \ldots \alpha^{l_{s} q_{s}(t)} \ldots \alpha^{l_{k} q_{k}(t)}=\alpha^{l_{1} q_{1}(t)} \alpha^{l_{2} q_{2}(t)} \ldots \beta^{l_{s} p} \ldots \alpha^{l_{k} q_{k}(t)}
$$

$\mathrm{e}$

$$
\alpha^{q_{s}(t)}=\alpha^{p l_{1} q_{1}(t)} \alpha^{p l_{2} q_{2}(t)} \ldots \alpha^{p l_{s} q_{s}(t)} \ldots \alpha^{p l_{k} q_{k}(t)} .
$$

Pela independência linear, devemos ter $\alpha^{q_{s}(t)\left(1-p l_{s}\right)}=e$, ou seja, $p l_{s}=1$, um absurdo. 
3.3 Não existência de representação de grau primo para um grupo abeliano livre de rank infinito

Segue o corolário:

Corolário 3.3.4. Um grupo abeliano livre A de rank infinito não pode ser transitivo e fechado por estado de grau $p$, com $p$ primo.

Demonstração. Suponha, por absurdo, que $A$ é transitivo fechado por estado de grau $p$. Seja $i$ como acima. Pelo Teorema 3.3.2, $A$ é conjugado para $D_{p}(i)$ e, pelo Teorema 3.3.3, $A$ deve ter rank menor ou igual a $i$, uma contradição.

Duas questões, que penso naturais, são: Existe uma representação fechada por estado de grau $m$ de um grupo abeliano livre de rank infinito, onde $m$ não é primo? Existe uma representação fechada por estado de grau $p$ primo de um produto semidireto $G=A \rtimes L$, onde $A$ é abeliano livre de rank infinito e $L$ é finito? Ainda não temos respostas para essas duas questões, mas a segunda vale para grau composto, como mostra o exemplo abaixo.

Exemplo 3.3.5. Seja $G=\left(\oplus_{i=1}^{\infty} C\right) \rtimes C_{2}=\left(\oplus_{i=1}^{\infty}\left\langle a_{i}\right\rangle\right) \rtimes\langle x\rangle$, onde a ação de $x$ sobre $G$ é dada por $a_{1}^{x}=a_{2}, a_{1}^{x}=a_{1}$ e $a_{i}^{x}=a_{i}$, com $i>2$. Então existe uma representação fechada por estado de grau 4 de G. De fato, seja

$$
H=\left\langle a_{1}^{2}, a_{i} \mid i=2,3,4, \ldots\right\rangle
$$

e $f: H \rightarrow G$ o homomorfismo que estende a função

$$
a_{1}^{2} \mapsto a_{2}, a_{2} \mapsto a_{1}, a_{3} \mapsto a_{2}, \ldots
$$

Note que $[G: H]=4$. Seja $a=a_{1}^{r_{1}} \ldots a_{n}^{r_{n}} \in H$. Assim existe s tal que ou $a^{f^{s}} \notin H$ ou $a^{f^{s}}=a_{1}^{2 l} a_{2}^{-l}$, para algum número inteiro l, ou seja, $a^{f^{s+1}}=1$. Mas $\left(a_{1}^{2 l} a_{2}^{-l}\right)^{x}=a_{1}^{-l} a_{2}^{2 l} e\left(a_{1}^{-l} a_{2}^{2 l}\right)^{f^{k}} \notin H$, para algum inteiro positivo $k$. Logo $f$ é um endomorfismo simples. A representação de G induzida por $f$ é dada por

$$
\begin{gathered}
x \mapsto \sigma=(02)(13), a_{1} \mapsto \alpha_{1}=\left(e, \alpha_{1}, \alpha_{1}, \alpha_{1}\right)(01), \\
a_{2} \mapsto \alpha_{2}=\left(\alpha_{1}, \alpha_{1}, e, \alpha_{1}\right)(23), a_{i} \mapsto \alpha_{i}=\left(\alpha_{i-1}, \alpha_{i-1}, \alpha_{i-1}, \alpha_{i-1}\right), i>2 .
\end{gathered}
$$

Note que o grupo gerado por $\alpha_{1}, \alpha_{2}, \ldots, \alpha_{n}, \ldots$ é fechado por estado e isomorfo a $\oplus_{i=1}^{\infty}\left\langle a_{i}\right\rangle$ e, portanto, é um abeliano livre de rank infinito fechado por estado de grau 4 .

Em [4, página 484], A. M. Brunner e S. N. Sidki constroem um exemplo de um grupo abeliano livre de rank infinito fechado por estado de grau 2, mas como no exemplo acima, esse grupo também não é transitivo. 


\subsection{Não existência de representação de grau primo para $C \imath C$}

Nesta seção é provado que $C \imath C$ não possui uma representação de grau primo. A demonstração de tal fato se seguirá em três proposições.

Seja $G=C \curlywedge C=\langle a\rangle$ 々 $\langle x\rangle$. Suponha que exista um subgrupo $H$ de $G$ com índice primo $p$ e $f: H \rightarrow G$ um endomorfismo simples.

Proposição 3.4.1. O grupo $A=\langle a\rangle^{\langle x\rangle}$ não é um subgrupo de $H$.

Demonstração. Suponha por absurdo que exista. Neste caso, $H=A\left\langle x^{p}\right\rangle$. Como $f$ é simples, $A^{f}$ não é subgrupo de $A$. Assim $A \cap A^{f}$ é trivial. De fato, seja $b$ um elemento não trivial de $A^{f} \cap A$. Suponha que $c x^{n} \in A^{f}$, onde $n$ é não nulo, com $c \in A$. Então $b c x^{n}$ não é igual a $c x^{n} b=b^{x^{-n}} c x^{n}$, ou seja, $b$ e $c x^{n}$ não comutam, absurdo. Daí, $A^{f} \leqslant A$, o que é uma contradição. Considere um inteiro não negativo $r$ tal que $A A^{f} \cap X=\left\langle x^{r}\right\rangle$. Logo existe $c \in A \operatorname{com} c^{f}=c^{\prime} x^{r} \mathrm{e}$

$$
A^{f}=\left\langle c^{\prime} x^{r}\right\rangle
$$

Com isso, se $u, v, c_{1}, c_{2} \in A$ são tais que $u^{f}=c_{1} x^{l r}$ e $v^{f}=c_{2} x^{l r}$, então $c_{1}=c_{2}$ e $u^{f}=v^{f}$. Sejam $\bar{c}, \bar{b} \in A$ e $k, l$ inteiros tais que $a^{f}=\bar{c} x^{k}$ e $\left(x^{p}\right)^{f}=\bar{b} x^{l}$. Como $\left(a^{f}\right)^{\left(x^{p}\right)^{f}}=\left(a^{x^{p}}\right)^{f} \mathrm{e}$

$$
\left(a^{f}\right)^{\left(x^{p}\right)^{f}}=\left(\bar{c} x^{k}\right)^{\bar{b} x^{l}}=\left(\bar{c}^{x^{l}}\left[\bar{b}^{x^{l}}, x^{-k}\right]\right) x^{k},
$$

então $\left(a^{x^{p}}\right)^{f}=a^{f}$. Analogamente, $\left(a^{x^{s+p}}\right)^{f}=\left(a^{x^{s}}\right)^{f}$, para todo $s \in \mathbb{Z}$. Daí, o subgrupo

$$
\left\langle a^{x^{s}-x^{s+p}} \mid s \in \mathbb{Z}\right\rangle^{X}
$$

é um subgrupo de $k e r(f) \cap H$, normal em $G$ e $f$-invariante. Absurdo.

Na proposição seguinte usamos a Proposição 2.1.5 para mostrar que $A_{0}^{f}$ não é um subgrupo de $A$.

Proposição 3.4.2. Se $A_{0}=H \cap A$, com $A=\langle a\rangle^{\langle x\rangle}$, então $A_{0}^{f}$ não é um subgrupo de A.

Demonstração. Suponha, por absurdo, que $A_{0}^{f}$ é subgrupo de $A$. Como $A$ não é subgrupo de $H, H$ se projeta sobrejetivamente sobre $\langle x\rangle$. Pela Proposição 2.1.5, 
$f$ pode ser considerada de tal forma que $\langle x\rangle^{f}$ é subgrupo de $\langle x\rangle$. Logo, se $K$ é um subgrupo de $A_{0}$ e $f$-invariante, então

$$
\left(K^{\langle x\rangle}\right)^{f}=\left(K^{f}\right)^{\langle x\rangle^{f}} \leqslant K^{\langle x\rangle}
$$

ou seja, $K^{\langle x\rangle}$ é um subgrupo de $H$ normal em $G$ e $f$-invariante, assim, $K=\{1\}$, pois $f$ é simples. Portanto, $f: A_{0} \rightarrow A$ é simples, com $\left[A: A_{0}\right]=p$, uma contradição ao Corolário 3.3.4.

Assim temos:

\section{Proposição 3.4.3. O grupo $A_{0}^{f} \cap A$ é trivial.}

Demonstração. Com efeito, sejam $b \in A_{0}^{f} \cap A$ um elemento não trivial e $c x^{n} \in$ $A_{0}^{f}$, onde $n$ é não nulo, $\operatorname{com} c \in A$. Assim $b c x^{n}$ não é igual a $c x^{n} b=b^{x^{-n}} c x^{n}$, ou seja, $b$ e $c x^{n}$ não comutam, absurdo.

Logo $\operatorname{ker}(f) \cap A_{0}$ é não trivial, pois $A_{0}$ é um abeliano livre de rank infinito e $A_{0}^{f}$ é abeliano de rank 1 . Tome $g \in \operatorname{ker}(f) \cap A_{0}$. Como $A H \cap\langle x\rangle=\langle x\rangle$, segue que para cada inteiro $n$ existe $b_{n} \in A$ tal que $b_{n} x^{n} \in H$. Assim

$$
\left(g^{x^{n}}\right)^{f}=\left(g^{b_{n} x^{n}}\right)^{f}=\left(g^{f}\right)^{\left(b_{n} x^{n}\right)^{f}}=1^{\left(b_{n} x^{n}\right)^{f}}=1,
$$

ou seja, $\left(\operatorname{ker}(f) \cap A_{0}\right)^{\langle x\rangle}$ é um subgrupo de $H$, normal em $G$ e $f$-invariante, um absurdo. Podemos agora enunciar o seguinte teorema.

Teorema 3.4.4. $O$ grupo $C \nmid C$ não possui uma representação fechada por estado de grau primo $p$. 
CAPÍTULO 4

\section{Representações de $G_{p, d}(d>1)$ de grau $p^{2}$}

Os resultados deste capítulo se encontram em [7]. Aqui demonstramos que $G_{p, d}=$ $C_{p} \prec C^{d}$ possui uma representação fechada por estado de grau $p^{2}$. Para tanto, após escolhermos um subgrupo adequado $H$ de índice $p^{2}$ em $G_{p, d}$, definimos um endomorfismo $f: H \rightarrow G$ e, então, demonstramos que $f$ é simples.

\subsection{Endomorfismo de grau $p^{2}$ em $G_{p, d}$}

Considere

$$
G_{p, d}=C_{p} \prec C^{d}=\langle a\rangle \prec\left\langle x_{1}, x_{2}, \ldots, x_{d}\right\rangle, X=\left\langle x_{1}, x_{2}, \ldots, x_{d}\right\rangle, A=\langle a\rangle^{X} .
$$

Se $H=G^{\prime} Y, \operatorname{com} Y=\left\langle x_{1}^{p}, x_{2}, \ldots, x_{d}\right\rangle$, então $[G: H]=p^{2}$. Mais que isso, $H$ é um subgrupo normal de $G$, $\operatorname{com}|G / H|=p^{2}$. Assim

$$
A_{0}=A \cap H=G^{\prime}=\left\langle a^{x_{1}-1}, a^{x_{2}-1}, \ldots, a^{x_{d}-1}\right\rangle^{X}
$$

é um subgrupo normal de $G_{p, d}$. Denote também $Z=\left\langle X_{2}\right\rangle$, onde $X_{2}=\left\{x_{2}, \ldots, x_{d}\right\}$. A ideia aqui é definir $f$ de tal forma que $f=\alpha: Y \rightarrow X, f=\mu: A_{0} \rightarrow A$ e satisfaça a relação

$$
\left(a^{u v} y\right)^{f}=a^{(u v)^{\mu}} y^{\alpha}=a^{u^{\alpha} v^{\mu}} y^{\alpha}
$$


para todos $y \in Y, u \in \mathcal{B}=\mathbb{K}\langle Y\rangle$ e $v \in \mathcal{I}=\left\langle x_{1}^{p}-1, x_{2}-1, \ldots, x_{d}-1\right\rangle$, onde $\mathcal{I}$ é olhado como um ideal de $\mathcal{A}=\mathbb{K}\langle X\rangle$ e $\mathbb{K}$ é o corpo com $p$ elementos. Note que $\alpha$ aplicado em $u$ é a extensão $\alpha: \mathcal{B} \rightarrow \mathcal{A}$ de $\alpha: Y \rightarrow X$. Como no Capítulo 2, vamos usar a correspondência $\mathcal{A}$ para $A$ e $\mathcal{I}$ para $A_{0}$. O intuito dessa correspondência é, para mais a frente, usar a Proposição 2.2.1 para demonstrar que $f$ é simples.

Defina $\alpha: Y \rightarrow X$ como o homomorfismo que estende a função

$$
x_{1}^{p} \mapsto x_{2}, x_{2} \mapsto x_{3}, \ldots, x_{d-1} \mapsto x_{d}, x_{d} \mapsto x_{1} .
$$

Note que $\alpha$ é um monomorfismo e um endomorfismo simples.

Dados um anel $R$ e um grupo $G$, podemos considerar o $R$-módulo $R[G]$, onde

$$
\sum_{g \in G} r_{g} g+\sum_{g \in G} s_{g} g=\sum_{g \in G}\left(r_{g}+s_{g}\right) g
$$

e

$$
r\left(\sum_{g \in G} r_{g} g\right)=\sum_{g \in G} r r_{g} g,
$$

para todos $\sum_{g \in G} r_{g} g, \sum_{g \in G} s_{g} g \in R[G]$ e todo $r \in R$. Defina $\phi: R[G] \rightarrow R$ dado por

$$
\left(\sum_{g \in G} r_{g} g\right) \phi=\sum_{g \in G} r_{g} .
$$

Não é difícil ver que $\phi$ é um $R$-homomorfismo de módulos. O núcleo $k e r(\phi)$ é o $R$-módulo livre gerado por

$$
\{g-1 ; g \in G \backslash\{1\}\} .
$$

Chamaremos $\operatorname{ker}(\phi)$ de ideal de aumento do $R$-módulo $R[G]$ e denotaremos tal ideal por $R[G]^{\prime}$.

Dito isso, vamos decompor os elementos de $\mathcal{I}$ de maneira única utilizando o conceito de ideal de aumento. Primeiro note que

$$
\mathbb{K}\langle x\rangle^{\prime}=\mathbb{K}\left\langle x^{p}\right\rangle^{\prime} \oplus \sum_{1 \leq i \leq p-1} \mathbb{K}\left\langle x^{p}\right\rangle\left(x^{i}-1\right) .
$$

Assim

$$
\mathcal{I}=\sum\left\{\mathbb{K}(z x-1) \mid x \in\left\langle x_{1}\right\rangle, z \in Z\right\}=
$$




$$
=\sum_{x \in\left\langle x_{1}\right\rangle} \mathbb{K}(x-1) \oplus \sum_{z \in Z} \mathbb{K}(z-1) \oplus \sum_{x \in\left\langle x_{1}\right\rangle, z \in Z} \mathbb{K}(x-1)(z-1)
$$

e substituindo

$$
\mathbb{K}\left\langle x_{1}\right\rangle^{\prime}=\sum_{x \in\left\langle x_{1}\right\rangle} \mathbb{K}(x-1)=\mathbb{K}\left\langle x_{1}^{p}\right\rangle^{\prime} \oplus \sum_{1 \leq i \leq p-1} \mathbb{K}\left\langle x_{1}^{p}\right\rangle\left(x_{1}^{i}-1\right)
$$

temos

$$
\begin{gathered}
\mathcal{I}=\mathbb{K}\left\langle x_{1}^{p}\right\rangle^{\prime} \oplus \sum_{1 \leq i \leq p-1} \mathbb{K}\left\langle x_{1}^{p}\right\rangle\left(x_{1}^{i}-1\right) \oplus \sum_{z \in Z} \mathbb{K}(z-1) \oplus \\
\mathbb{K}\left\langle x_{1}^{p}\right\rangle^{\prime}(z-1) \oplus \sum_{z \in Z} \sum_{1 \leq i \leq p-1} \mathbb{K}\left\langle x_{1}^{p}\right\rangle\left(x_{1}^{i}-1\right)(z-1) .
\end{gathered}
$$

Portanto, cada elemento $v \in \mathcal{I}$ pode ser escrito de maneira única na forma

$$
v=b_{0}+\sum_{1 \leq i \leq p-1} b_{i}\left(x_{1}^{i}-1\right)+\sum_{z \in Z} a_{z}(z-1)+\sum_{z \in Z} \sum_{1 \leq i \leq p-1} b_{i, z}\left(x_{1}^{i}-1\right)(z-1),
$$

onde $b_{0} \in \mathbb{K}\left\langle x_{1}^{p}\right\rangle^{\prime}$ e $a_{z}, b_{i}, b_{i, z} \in \mathbb{K}\left\langle x_{1}^{p}\right\rangle$.

Vamos então definir $\mu$ sobre $\mathcal{I}$. Primeiro defina

$$
\mu: b \mapsto 0, x_{1}^{i}-1 \mapsto i,
$$

para todo $b \in \mathcal{B}^{\prime}$ e todo $1 \leq i \leq p-1$. Como $\alpha$ se estende $\operatorname{de} \mathcal{B}$ em $\mathcal{A}$, podemos estender $\mu \operatorname{de} \mathcal{I}$ em $\mathcal{A}$ pondo

$$
\begin{gathered}
v^{\mu}=b_{0}^{\mu}+\sum_{1 \leq i \leq p-1} b_{i}^{\alpha}\left(x_{1}^{i}-1\right)^{\mu}+\sum_{z \in Z} a_{z}^{\alpha}(z-1)^{\mu}+\sum_{z \in Z} \sum_{1 \leq i \leq p-1} b_{i, z}^{\alpha}(z-1)^{\alpha}\left(x_{1}^{i}-1\right)^{\mu}= \\
=\sum_{1 \leq i \leq p-1} i b_{i}^{\alpha}+\sum_{z \in Z} \sum_{1 \leq i \leq p-1} i b_{i, z}^{\alpha}(z-1)^{\alpha},
\end{gathered}
$$

para todo $v \in \mathcal{I}$.

Proposição 4.1.1. A aplicação $f: H \rightarrow G_{p, d}$ induzida por $\alpha$ e $\mu$, definidas acima, é um homomorfismo bem definido.

Demonstração. Pela unicidade de escrita de cada elemento de $\mathcal{I}$, devemos apenas demonstrar que

$$
(v x)^{\mu}=\left(v^{\mu}\right) x^{\alpha}
$$

para todo $x \in Y$. Mas isso segue como um processo análogo ao feito no Exemplo 2.2.2. 
Passaremos a demonstrar que $f$ é simples. Tal fato seguirá após quatro lemas.

Lema 4.1.2. Sejam $u, i \geq 1 e 2 \leq j \leq d$. Escreva $u=u_{0}+u_{1}$ p e $i=i_{0}+i_{1} p$, onde $0 \leq u_{0}, i_{0} \leq p-1$. Então

$$
\begin{gathered}
\left(x_{1}^{u_{0}+u_{1} p}-1\right)^{\mu}=u_{0} x_{2}^{u_{1}},\left(x_{1}^{u}\left(x_{1}^{i}-1\right)\right)^{\mu}=\left(\left(u_{0}+i_{0}\right) x_{2}^{i_{1}}-u_{0}\right) x_{2}^{u_{1}}, \\
\left(x_{1}^{u}\left(x_{j}^{i}-1\right)\right)^{\mu}=u_{0} x_{2}^{u_{1}}\left(x_{j+1}^{i}-1\right),\left(x_{j}^{u}\left(x_{1}^{i}-1\right)\right)^{\mu}=i_{0} x_{j+1}^{u} x_{2}^{i_{1}},
\end{gathered}
$$

onde $j+1$ é tomado módulo $d$.

Demonstração. Note que $\left(x_{1}^{p}\left(x_{1}-1\right)\right)^{\mu}=x_{2} \cdot 1=x_{2}$. De maneira mais geral, temos

$$
\begin{aligned}
\left(x_{1}^{u_{0}+u_{1} p}-1\right)^{\mu}= & \left(\left(x_{1}^{u_{1} p}-1\right)\left(x_{1}^{u_{0}}-1\right)+\left(x_{1}^{u_{1} p}-1\right)+\left(x_{1}^{u_{0}}-1\right)\right)^{\mu}= \\
& =\left(x_{2}^{u_{0}}-1\right) u_{0}-0+u_{0}=u_{0} x_{2}^{u_{1}}
\end{aligned}
$$

e a primeira equação está verificada.

Agora, se $u_{0}+1 \leq p-1$, então

$$
x_{1}^{u_{0}+u_{1} p}\left(x_{1}-1\right)=x_{1}^{\left(u_{0}+1\right)+u_{1} p}-x_{1}^{u_{0}+u_{1} p}=\left(x_{1}^{\left(u_{0}+1\right)+u_{1} p}-1\right)-\left(x_{1}^{u_{0}+u_{1} p}-1\right),
$$

daí

$$
\left(x_{1}^{u_{0}+u_{1} p}\left(x_{1}-1\right)\right)^{\mu}=\left(u_{0}+1\right) x_{2}^{u_{1}}-u_{0} x_{2}^{u_{1}}=x_{2}^{u_{1}} .
$$

E se $u_{0}=p-1$, então

$$
x_{1}^{(p-1)+u_{1} p}\left(x_{1}-1\right)=\left(x_{1}^{\left(u_{1}-1\right) p}-1\right)-\left(x_{1}^{(p-1)+u_{1} p}-1\right),
$$

daí

$$
\left(x_{1}^{(p-1)+u_{1} p}\left(x_{1}-1\right)\right)^{\mu}=0-(p-1) x_{2}^{u_{1}}=x_{2}^{u_{1}} .
$$

De qualquer maneira, temos

$$
\left(x_{1}^{u}\left(x_{1}-1\right)\right)^{\mu}=x_{2}^{u_{1}},
$$

para todo $u$. Para $i$ qualquer temos

$$
\begin{gathered}
\left(x_{1}^{u}\left(x_{1}^{i}-1\right)\right)^{\mu}=\left(x_{1}^{(u+i)}-x_{1}^{u}\right)^{\mu}=\left(\left(x_{1}^{u+i}-1\right)-\left(x_{1}^{u}-1\right)\right)^{\mu}= \\
=(u+i)_{0} x_{2}^{(u+i)_{1}}-u_{0} x_{2}^{u_{1}}
\end{gathered}
$$


Como $(u+i)_{0}=u_{0}+i_{0}$, se $p \nmid\left(u_{0}+i_{0}\right)$, e $(u+i)_{0}=0$, se $p \mid\left(u_{0}+i_{0}\right)$, a segunda equação segue.

Para chegar na terceira e quarta equações tomamos $2 \leq j \leq d$ e fazemos, respectivamente,

$$
\begin{gathered}
\left(x_{1}^{u}\left(x_{j}^{i}-1\right)\right)^{\mu}=\left(\left(x_{1}^{u}-1\right)\left(x_{j}^{i}-1\right)-\left(x_{j}^{i}-1\right)\right)^{\mu}=\left(\left(x_{j}^{i}-1\right)\left(x_{1}^{u}-1\right)\right)^{\mu}= \\
=\left(x_{j+1}^{i}-1\right) u_{0} x_{2}^{u_{1}}
\end{gathered}
$$

$\mathrm{e}$

$$
\left(x_{j}^{i}\left(x_{1}^{i}-1\right)\right)^{\mu}=\left(x_{j+1}^{u}\right) i_{0} x_{2}^{i_{1}}=i_{0} x_{j+1}^{u} x_{2}^{i_{1}} .
$$

Lema 4.1.3. Sejam $q(x)=c_{0}+c_{1} x+\ldots+c_{s} x^{s} \in \mathbb{K}[x] e 0 \leq u=u_{0}+u_{1} p$, com $0 \leq u_{0} \leq p-1$. Suponha que $q\left(x_{1}\right) \in \mathcal{I}$. Então $\sum_{0 \leq i \leq s} c_{i}=0 e$

$$
q\left(x_{1}\right)^{\mu}=\sum_{1 \leq i \leq s} c_{i} i_{0} x_{2}^{i_{1}}
$$

Além disso,

$$
\left(x_{1}^{u} q\left(x_{1}\right)\right)^{\mu}-x_{2}^{u_{1}} q\left(x_{1}\right)^{\mu}=u_{0}\left(\sum_{1 \leq i \leq s} c_{i}\left(x_{2}^{i_{1}}-1\right)\right) x_{2}^{u_{1}} .
$$

Demonstração. Temos que

$q(x)=c_{0}+c_{1} x+\ldots+c_{s} x^{s}=\left(c_{0}+c_{1}+\ldots+c_{s}\right)+\left(c_{1}(x-1)+\ldots+c_{s}\left(x^{s}-1\right)\right)$.

Logo $q\left(x_{1}\right) \in \mathcal{I}$ se, e somente se, $c_{0}+c_{1}+\ldots+c_{s}=0$. Daí $q\left(x_{1}\right) \in \mathcal{I}$ implica que

$$
q\left(x_{1}\right)^{\mu}=\left(\sum_{0 \leq i \leq s} c_{i}\left(x_{1}^{i}-1\right)\right)^{\mu}=\sum_{1 \leq i \leq s} c_{i} i_{0} x_{2}^{i_{1}} .
$$

Para a segunda equação temos

$$
\begin{gathered}
\left(x_{1} q\left(x_{1}\right)\right)^{\mu}=\left(\sum_{0 \leq i \leq s} c_{i}\left(x_{1}^{u}\left(x_{1}^{i}-1\right)\right)\right)^{\mu}= \\
=\sum_{0 \leq i \leq s} c_{i}\left(\left(u_{0}+i_{0}\right) x_{2}^{i_{1}}-u_{0}\right) x_{2}^{u_{1}}=\left(\sum_{0 \leq i \leq s} c_{i} u_{0} x_{2}^{i_{1}}+c_{i} i_{0} x_{2}^{i_{1}}-c_{i} u_{0}\right) x_{2}^{u_{1}}=
\end{gathered}
$$




$$
\begin{gathered}
=\left(u_{0} \sum_{0 \leq i \leq s} c_{i}\left(x_{2}^{i_{1}}+1\right)+\sum_{0 \leq i \leq s} c_{i} i_{0} x_{2}^{i_{1}}\right) x_{2}^{u_{1}}= \\
=\left(u_{0} \sum_{0 \leq i \leq s} c_{i}\left(x_{2}^{i}+1\right)+q\left(x_{1}\right)^{\mu}\right) x_{2}^{u_{1}} .
\end{gathered}
$$

Portanto

$$
\left(x_{1}^{u} q\left(x_{1}\right)\right)^{\mu}-x_{2}^{u_{1}} q\left(x_{1}\right)^{\mu}=u_{0}\left(\sum_{1 \leq i \leq s} c_{i}\left(x_{2}^{i_{1}}-1\right)\right) x_{2}^{u_{1}} .
$$

Como desejado.

Lema 4.1.4. Se $\mathcal{K}$ é um ideal de $\mathcal{A}$ contido em $\mathcal{I}$ e $\mu$-invariante e $q\left(x_{j}\right) \in \mathcal{K}$, para algum $j$, então $q\left(x_{j}\right)=0$.

Demonstração. Seja $q(x)=c_{0}+c_{1} x+\ldots+c_{s} x^{s} \neq 0$. Defina $e(q(x))=\left\{i \mid c_{i} \neq 0\right\}$ e $\lambda(q(x))=\sum_{i \in e(q(x))} i$. Escrevendo $0 \leq i=i_{0}+i_{1} p$, com $0 \leq i_{0} \leq p-1$, segue que

$$
\lambda(q(x))=\sum_{i \in e(q(x))} i_{0}+\left(\sum_{i \in e(q(x))} i_{1}\right) p .
$$

Suponha agora que exista um polinômio não nulo $q(x)$ tal que $q\left(x_{j}\right) \in \mathcal{K}$, para algum $j$. Escolha $q(x) \operatorname{com} \lambda(q(x))$ mínimo. Podemos assumir que $c_{0} \neq 0$.

Suponha que $j=1$, ou seja, $q\left(x_{1}\right) \in \mathcal{K}$. Escolha $u=u_{0}+u_{1} p$, com $0<u_{0} \leq$ $p-1$. Pelo lema anterior,

$$
\left(x_{1}^{u} q\left(x_{1}\right)\right)^{\mu}-x_{2}^{u_{1}} q\left(x_{1}\right)^{\mu}=u_{0}\left(\sum_{1 \leq i \leq s} c_{i}\left(x_{2}^{i_{1}}-1\right)\right) x_{2}^{u_{1}} \in \mathcal{K} .
$$

Logo $l\left(x_{2}\right)=\sum_{1 \leq i \leq s} c_{i}\left(x_{2}^{i_{1}}-1\right) \in \mathcal{K}$ e $\lambda(l(x)) \leq \sum_{i \in e(q(x))} i_{1}$. Daí ou $\lambda(q(x))=$ $\lambda(l(x))$ ou $\lambda(l(x))=0$. No primeiro caso,

$$
\sum_{i \in e(q(x))} i_{0}+\left(\sum_{i \in e(q(x))} i_{1}\right) p \leq \sum_{i \in e(q(x))} i_{1},
$$


ou ainda

$$
\sum_{i \in e(q(x))} i_{0} \leq\left(\sum_{i \in e(q(x))} i_{1}\right)(1-p) .
$$

Assim

$$
\sum_{i \in e(q(x))} i_{0}=\sum_{i \in e(q(x))} i_{1}=0
$$

e $q\left(x_{1}\right)=c_{0} \in \mathcal{K}$. Mas, $\mathcal{K}$ é um ideal de $\mathcal{I}, \log c_{0}=0$, um absurdo.

No segundo caso, $0=l\left(x_{2}\right)=\left(l\left(x_{1}\right)\right)^{\alpha}$. Mas $\alpha$ é um monomorfismo, logo $l\left(x_{1}\right)=0$. Defina $L_{j}=\left\{i \in e(q(x)) \mid i_{1}=j\right\}$ e seja $t$ tal que $p^{t} \leq s<p^{t+1}$. Então,

$$
\begin{gathered}
l\left(x_{1}\right)=\sum_{1 \leq i \leq s} c_{i}\left(x_{1}^{i_{1}}-1\right)= \\
=\sum_{i \in L_{0}} c_{i}\left(x_{1}^{i_{1}}-1\right)+\sum_{i \in L_{1}} c_{i}\left(x_{2}^{i_{1}}-1\right)+\ldots+\sum_{i \in L_{t}} c_{i}\left(x_{2}^{i_{1}}-1\right)= \\
=\sum_{i \in L_{1}} c_{i}\left(x_{1}^{i_{1}}-1\right)+\sum_{i \in L_{2}} c_{i}\left(x_{2}^{i_{1}}-1\right)+\ldots+\sum_{i \in L_{t}} c_{i}\left(x_{2}^{i_{1}}-1\right)= \\
=\left(\sum_{1 \leq i \leq p-1} c_{i}\right)\left(x_{1}-1\right)+\left(\sum_{1 \leq i \leq p-1} c_{p+i}\right)\left(x_{1}^{2}-1\right)+\ldots+\left(\sum_{1 \leq i \leq p-1} c_{p^{t}+i}\right)\left(x_{1}^{s}-1\right) .
\end{gathered}
$$

Como $l\left(x_{1}\right)=0$ segue que

$$
\sum_{1 \leq i \leq p-1} c_{i}=\sum_{1 \leq i \leq p-1} c_{p+i}=\ldots=\sum_{1 \leq i \leq p-1} c_{p^{t}+i}=0 .
$$

Desde que $\sum_{0 \leq i \leq p-1} c_{i}=0$, temos $c_{0}=0$, uma contradição.

Agora, se $2 \leq j \leq d$, então $q\left(x_{j}\right)\left(x_{1}^{u}-1\right) \in \mathcal{K}$. Daí

$$
\left(q\left(x_{j}\right)\left(x_{1}^{u}-1\right)\right)^{\mu}=q\left(x_{j+1}\right) u_{0} x_{2}^{u_{1}} \in \mathcal{K}
$$

e $q\left(x_{j+1}\right) \in \mathcal{K}$. O que nos leva a afirmar que $q\left(x_{1}\right) \in \mathcal{K}$.

Lema 4.1.5. Se $\mathcal{K}$ é um ideal de $\mathcal{A}$ contido em $\mathcal{I}$ e $\mu$-invariante, então $\mathcal{K}=\{0\}$.

Demonstração. Suponha, por absurdo, que $\mathcal{K} \neq\{0\}$. Seja $w=w\left(x_{j_{0}}, x_{j_{1}}, \ldots, x_{j_{t}}\right)$ em $\mathcal{K}$ tal que

i) os expoentes de cada $x_{j_{i}}$ são positivos; 
ii) $w$ possui $\delta(w)$ mínimo, onde

$$
\delta(w)=\sum_{1 \leq i \leq d} \delta_{i}(w)
$$

com $\delta_{i}(w)$ o $x_{i}$ grau de $w$;

iii) $x_{j_{0}}=x_{1}$.

Como $\mathcal{K}$ é um ideal e por um argumento análogo ao feito no final da demonstração do lema anterior, podemos tomar $w$ com as três hipóteses acima.

Pelo lema anterior, podemos escrever $w$ da forma

$w=\sum_{0 \leq i \leq s} w_{i}\left(x_{j_{1}}, \ldots, w_{j_{t}}\right) x_{1}^{i}=\sum_{0 \leq i \leq s} w_{i}\left(x_{j_{1}}, \ldots, w_{j_{t}}\right)+\sum_{1 \leq i \leq s} w_{i}\left(x_{j_{1}}, \ldots, w_{j_{t}}\right)\left(x_{1}^{i}-1\right)$

onde $2 \leq j_{1}<\ldots<j_{t} \leq d$ e $t>0$. Então

$$
\delta(w)=s+\sum_{j \neq 1} \delta_{j}(w)
$$

Como $\sum_{1 \leq i \leq s} w_{i}\left(x_{j_{1}}, \ldots, w_{j_{t}}\right)\left(x_{1}^{i}-1\right) \in \mathcal{I}$, devemos ter $\sum_{0 \leq i \leq s} w_{i}\left(x_{j_{1}}, \ldots, w_{j_{t}}\right) \in$ $\mathcal{B}^{\prime}$. Daí

$$
w^{\mu}=\sum_{1 \leq i \leq s} w_{i}\left(x_{j_{1}}, \ldots, w_{j_{t}}\right)\left(i_{0} x_{2}^{i_{1}}\right)=\sum_{1 \leq i \leq s} w_{i}\left(x_{j_{1}}, \ldots, w_{j_{t}}\right) i x_{2}^{\left[\frac{i}{p}\right]} \in \mathcal{K}
$$

$\mathrm{e}$

$$
\delta\left(w^{\mu}\right)=\left[\frac{i}{p}\right]+\sum_{j \neq 1} \delta_{j}(w) \leq \delta(w)=s+\sum_{j \neq 1} \delta_{j}(w) .
$$

Pela minimalidade de $w$, segue que $w^{\mu}=0$ ou $1 \leq s \leq p-1$.

Suponha que $1 \leq s \leq p-1$. Vamos analisar agora o efeito da aplicação de $\mu$ em $W_{j}=w \cdot\left(x_{1}^{j}-1\right)$, onde $1 \leq j \leq p-1$. Inicialmente note que,

$$
\begin{gathered}
W_{j}=w \cdot\left(x_{1}^{j}-1\right)= \\
=\left(\sum_{0 \leq i \leq s} w_{i}\left(x_{j_{1}}, \ldots, w_{j_{t}}\right)+\sum_{1 \leq i \leq s} w_{i}\left(x_{j_{1}}, \ldots, w_{j_{t}}\right)\left(x_{1}^{i}-1\right)\right)\left(x_{1}^{j}-1\right)= \\
=\sum_{0 \leq i \leq s} w_{i}\left(x_{j_{1}}, \ldots, w_{j_{t}}\right)\left(x_{1}^{j}-1\right)+\sum_{1 \leq i \leq s} w_{i}\left(x_{j_{1}}, \ldots, w_{j_{t}}\right)\left(x_{1}^{i}-1\right)=
\end{gathered}
$$




$$
\begin{gathered}
=\sum_{0 \leq i \leq s} w_{i}\left(x_{j_{1}}, \ldots, w_{j_{t}}\right)\left(x_{1}^{j}-1\right)+ \\
+\sum_{1 \leq i \leq s} w_{i}\left(x_{j_{1}}, \ldots, w_{j_{t}}\right)\left(\left(x_{1}^{i+j}-1\right)-\left(x_{1}^{i}-1\right)-\left(x_{1}^{j}-1\right)\right) .
\end{gathered}
$$

Uma vez que $\left[\frac{j}{p}\right]=0$, temos

$$
\begin{gathered}
W_{j}^{\mu}=\left(\sum_{0 \leq i \leq s} w_{i}\left(x_{j_{1}}, \ldots, w_{j_{t}}\right)\left(x_{1}^{j}-1\right)\right)^{\mu}+ \\
+\left(\sum_{1 \leq i \leq s} w_{i}\left(x_{j_{1}}, \ldots, w_{j_{t}}\right)\left(\left(x_{1}^{i+j}-1\right)-\left(x_{1}^{i}-1\right)-\left(x_{1}^{j}-1\right)\right)\right)^{\mu}= \\
=\sum_{0 \leq i \leq s} w_{i}\left(x_{j_{1}+1}, \ldots, w_{j_{t}+1}\right) j_{0} x_{2}^{\left[\frac{j}{p}\right]}+ \\
+\sum_{1 \leq i \leq s} w_{i}\left(x_{j_{1}}, \ldots, w_{j_{t}}\right)\left((i+j)_{0} x_{2}^{\left[\frac{i+j}{p}\right]}-i_{0} x_{2}^{\left[\frac{i}{p}\right]}-j_{0} x_{2}^{\left[\frac{j}{p}\right]}\right)= \\
=\sum_{0 \leq i \leq s} w_{i}\left(x_{j_{1}+1}, \ldots, w_{j_{t}+1}\right) j-\sum_{1 \leq i \leq s} w_{i}\left(x_{j_{1}+1}, \ldots, w_{j_{t}+1}\right) j+ \\
+\sum_{1 \leq i \leq s} w_{i}\left(x_{j_{1}}, \ldots, w_{j_{t}}\right)(i+j)_{0} x_{2}^{\left[\frac{i+j}{p}\right]}-\sum_{1 \leq i \leq s} w_{i}\left(x_{j_{1}}, \ldots, w_{j_{t}}\right) i_{0} x_{2}^{\left[\frac{i}{p}\right]}= \\
=w_{0}\left(x_{j_{1}+1}, \ldots, w_{j_{t}+1}\right) j+\sum_{1 \leq i \leq s} w_{i}\left(x_{j_{1}}, \ldots, w_{j_{t}}\right)(i+j) x_{2}^{\left[\frac{i+j}{p}\right]}- \\
-\sum_{1 \leq i \leq s} w_{i}\left(x_{j_{1}}, \ldots, w_{j_{t}}\right) i x_{2}^{\left[\frac{i}{p}\right]} .
\end{gathered}
$$

De $\sum_{1 \leq i \leq s} w_{i}\left(x_{j_{1}}, \ldots, w_{j_{t}}\right) i x_{2}^{\left[\frac{i}{p}\right]} \in \mathcal{K}$, para $1 \leq j \leq p-1$ segue que

$$
V_{j}=w_{0}\left(x_{j_{1}+1}, \ldots, w_{j_{t}+1}\right) j+\sum_{1 \leq i \leq s} w_{i}\left(x_{j_{1}+1}, \ldots, w_{j_{t}+1}\right)(i+j) x_{2}^{\left[\frac{i+j}{p}\right]} \in \mathcal{K} .
$$

Suponha que $V_{j} \neq 0$, para algum $j$. Então

$$
\delta(w)=s+\sum_{j \neq 1} \delta_{j}(w) \leq \delta\left(V_{j}\right) \leq\left[\frac{s+j}{p}\right]+\sum_{j \neq 1} \delta_{j}(w),
$$


ou seja

$$
s \leq\left[\frac{s+j}{p}\right] .
$$

Escreva $s=s_{0}+s_{1} p$, onde $0 \leq s_{0} \leq p-1$, assim

$$
s=s_{0}+s_{1} p \leq\left[\frac{s+j}{p}\right] \leq\left[\frac{s_{0}+s_{1} p+p-1}{p}\right]=\left[\frac{s_{0}-1+\left(s_{1}+1\right) p}{p}\right] .
$$

Se $s_{0}=0$, segue que $s_{1} p \leq s_{1}$, ou seja, $s_{1}=0$ e $s=0$, o que não ocorre. Se $0<s_{0} \leq p-1$, então $s_{1}(p-1) \leq 1-s_{0} \leq 0$ e $s_{0}=1, s_{1}=0$. Daí $s=1$. Neste caso, $w=w_{0}\left(x_{j_{1}}, \ldots, x_{j_{t}}\right)+w_{1}\left(x_{j_{1}}, \ldots, x_{j_{t}}\right) x_{1}$, com $w_{0}$ e $w_{1}$ ambos não nulos. Como $w^{\mu}=w_{1}^{\alpha}=w_{1}\left(x_{j_{1}+1}, \ldots, x_{j_{t}+1}\right) \in \mathcal{K}$, pela minimalidade de $w$, segue que $w_{1}=0$, uma contradição.

Com isso, podemos supor que $V_{j}=0$, para todo $j=1, \ldots, p-1$. Aplicando $\alpha^{-1}$ temos que $\left(V_{j}\right)^{\alpha^{-1}}=0$, para todo $j=1, \ldots, p-1$. Em particular

$$
\begin{gathered}
\left(V_{p-1}\right)^{\alpha^{-1}}=w_{0}\left(x_{j_{1}}, \ldots, w_{j_{t}}\right)(p-1)+\sum_{1 \leq i \leq s} w_{i}\left(x_{j_{1}}, \ldots, w_{j_{t}}\right)(i+p-1) x_{1}^{\left[\frac{i+p-1}{p}\right] p}= \\
=-w_{0}\left(x_{j_{1}}, \ldots, w_{j_{t}}\right)+\sum_{1 \leq i \leq s} w_{i}\left(x_{j_{1}}, \ldots, w_{j_{t}}\right)(i+p-1) x_{1}^{\left[\frac{i+p-1}{p}\right] p}=0 .
\end{gathered}
$$

Portanto, $w_{0}=0$, o que é um absurdo.

Fica assim demonstrado o seguinte teorema:

Teorema 4.1.6. Seja $\left.G_{p, d}=C_{p}\right\rangle C^{d}=\langle a\rangle\left\langle\left\langle x_{1}, x_{2}, \ldots, x_{d}\right\rangle\right.$, com $d \geq 1$. Considere $H=G^{\prime} Y$, onde $Y=\left\langle x_{1}^{p}, x_{2}, \ldots, x_{d}\right\rangle$. Então a função

$$
\begin{gathered}
a^{x_{1}-1} \mapsto a^{i}, a^{x_{1}^{2}-1} \mapsto a^{2}, \ldots, a^{x_{1}^{p-1}-1} \mapsto a^{p-1}, a^{z-1} \mapsto 1, \forall z \in Y, \\
x_{1}^{p} \mapsto x_{2}, x_{2} \mapsto x_{3}, \ldots, x_{d-1} \mapsto x_{d}, x_{d} \mapsto x_{1},
\end{gathered}
$$

estende-se a um endomorfismo $f: H \rightarrow G_{p, d}$ simples. 


\subsection{Representação fechada por estado de grau $p^{2}$ de $G_{p, d}$}

Lembrando que $H=G^{\prime} Y$ tem índice $p^{2}$ em $G_{p, d}$, vamos agora escolher um transversal $T$ de $H$ em $G$ e, assim, explicitar a representação de $G_{p, d}$ de grau $p^{2}$ induzida por $f$ e $T$. Defina

$$
T=\left\{x_{1}^{i} a^{j} \mid 0 \leq i, j \leq p-1\right\},
$$

ou seja, $T$ é o seguinte transversal de $H$ em $G$

$$
T=\left\{a, a^{2}, \ldots, a^{p-1}, x_{1}, x_{1} a, \ldots, x_{1} a^{p-1}, \ldots, x_{1}^{p-1}, x_{1}^{p-1} a, \ldots, x_{1}^{p-1} a^{p-1}\right\} .
$$

Note que

$$
\begin{gathered}
H x_{1}^{i} a^{j} a=H x_{1}^{i} a^{j+1}, j+1(\bmod p), \\
H x_{1}^{i} a^{j} x_{1}=H x_{1}^{i+1} a^{j x_{1}}=H x_{1}^{i+1} a^{j x_{1}-j} a^{j}=H x_{1}^{i+1} a^{j}, i+1(\bmod p), \\
H x_{1}^{i} a^{j} x_{l}=H x_{1}^{i} a^{j}, 2 \leq l \leq d .
\end{gathered}
$$

Ou seja, se $\sigma(g)$ é a permutação que $g \in G_{p, d}$ induz em $T$, então

$$
\begin{gathered}
\sigma(a): x_{1}^{i} a^{j} \mapsto x_{1}^{i} a^{j+1}, j+1(\bmod p), \\
\sigma\left(x_{1}\right): x_{1}^{i} a^{j} \mapsto x_{1}^{i+1} a^{j}, i+1(\bmod p), \\
\sigma\left(x_{l}\right): x_{1}^{i} a^{j} \mapsto x_{1}^{i} a^{j}, 2 \leq l \leq d,
\end{gathered}
$$

em notação de permutações temos

$$
\begin{gathered}
\sigma(a)=(0 \ldots p-1)(p \ldots 2 p-1) \ldots\left(p(p-1) \ldots p^{2}-1\right) \\
\sigma\left(x_{1}\right)=(0 p \ldots p(p-1))(1(p+1) \ldots p(p-1)+1) \ldots\left((p-1)(2 p-1) \ldots p^{2}-1\right), \\
\sigma\left(x_{l}\right)=e, 2 \leq l \leq d .
\end{gathered}
$$

Os cofatores $t_{k} g t_{k^{\sigma(g)}}^{-1}$, com $g \in G_{p, d}$ e $t_{k} \in T$, induzidos por $a, x_{1}$ e $x_{l}$, $2 \leq l \leq d$, são dados por:

i) cofatores induzidos por $a$.

$$
\left(x_{1}^{i} a^{j}\right) a\left(x_{1}^{i} a^{j+1}\right)^{-1}=1
$$

com $0 \leq j \leq p-2$, e

$$
\left(x_{1}^{i} a^{p-1}\right) a\left(x_{1}^{i}\right)^{-1}=1 .
$$


ii) cofatores induzidos por $x_{1}$.

$$
\left(x_{1}^{i} a^{j}\right) x_{1}\left(x_{1}^{i+1} a^{j}\right)^{-1}=x_{1}^{i} a^{j} x_{1} a^{-j} x_{1}^{-1-i}=\left(a^{j}\right)^{x_{1}^{-i}}\left(a^{j}\right)^{x_{1}^{-(i+1)}}=\left(a^{j}\right)^{x_{1}^{-i}\left(1-x_{1}^{-1}\right)},
$$

$\operatorname{com} 0 \leq i \leq p-2$, $\mathrm{e}$

$$
\left(x_{1}^{p-1} a^{j}\right) x_{1}\left(a^{j}\right)^{-1}=x_{1}^{p-1} a^{j} x_{1} a^{-j}=x_{1}^{p}\left(a^{j}\right)^{x_{1}-1}=\left(a^{j}\right)^{\left(x_{1}-1\right) x_{1}^{p}} x_{1}^{p} .
$$

iii) cofatores induzidos por $x_{l}, 2 \leq l \leq d$.

$$
\left(x_{1}^{i} a^{j}\right) x_{l}\left(x_{1}^{i} a^{j}\right)^{-1}=x_{1}^{i} a^{j} x_{l} a^{-j} x_{1}^{-i}=\left(a^{j}\right)^{x_{1}^{-1}\left(x_{l}-1\right) x_{l}^{-1}} x_{l} .
$$

Aplicando $f$ nesses cofatores temos

$$
\begin{gathered}
\left(a^{j}\right)^{x_{1}^{-i}\left(1-x_{1}^{-1}\right)} \mapsto\left(a^{j}\right)^{x_{2}^{-1}}, \\
\left(a^{j}\right)^{\left(x_{1}-1\right) x_{1}^{p}} x_{1}^{p} \mapsto\left(a^{j}\right)^{x_{2}^{-1}} x_{2}, \\
\left(a^{j}\right)^{x_{1}^{-1}\left(x_{l}-1\right) x_{l}^{-1}} x_{l} \mapsto\left(a^{j}\right)^{-i x_{2}^{-1} x_{l+1}^{-1}\left(x_{l+1}-1\right)} x_{l+1}, l+1(\bmod d) .
\end{gathered}
$$

Portanto, na representação $\varphi: G_{p, d} \rightarrow \mathcal{A}_{p^{2}}$, induzida por $f$ e $T$, temos

$$
\begin{gathered}
a^{\varphi}=\sigma(a), \\
x_{l}^{\varphi}=\left(x_{l 1}, x_{l 2}, \ldots, x_{l\left(p^{2}-1\right)}\right) \sigma\left(x_{l}\right),
\end{gathered}
$$

onde $x_{l t}$ é tal que $t=i p+j, 0 \leq j \leq p-1$, e

$$
\begin{gathered}
x_{1 t}=\left(a^{\varphi}\right)^{j\left(x_{2}^{\varphi}\right)^{-1}}, 0 \leq i \leq p-2, \\
x_{1 t}=\left(a^{\varphi}\right)^{j\left(x_{2}^{\varphi}\right)^{-1}} x_{2}^{\varphi}, i=p-1
\end{gathered}
$$

$\mathrm{e}$

$$
x_{l t}=\left(a^{\varphi}\right)^{-i j\left(x_{2}^{\varphi}\right)^{-1}\left(x_{l+1}^{\varphi}\right)^{-1}\left(x_{l+1}^{\varphi}-e\right)} x_{l+1}^{\varphi}, 2 \leq l \leq d, l+1(\bmod d) .
$$

Segue o teorema:

Teorema 4.2.1. Defina

$$
\begin{gathered}
\sigma=(0 \ldots p-1)(p \ldots 2 p-1) \ldots\left(p(p-1) \ldots p^{2}-1\right) \\
\sigma\left(\alpha_{1}\right)=(0 p \ldots p(p-1))(1(p+1) \ldots p(p-1)+1) \ldots\left((p-1)(2 p-1) \ldots p^{2}-1\right)
\end{gathered}
$$


$e$

$$
\sigma\left(\alpha_{l}\right)=e, 2 \leq l \leq d
$$

Sejam

$$
\alpha_{l}=\left(\alpha_{l 1}, \alpha_{l 2}, \ldots, \alpha_{l\left(p^{2}-1\right)}\right) \sigma\left(\alpha_{l}\right), l=1, \ldots, d
$$

onde $\alpha_{l t}$ é tal que $t=i p+j, 0 \leq j \leq p-1$, $e$

$$
\begin{gathered}
\alpha_{1 t}=\sigma^{j \alpha_{2}^{-1}}, 0 \leq i \leq p-2, \\
\alpha_{1 t}=\sigma^{j \alpha_{2}^{-1}} \alpha_{2}, i=p-1
\end{gathered}
$$

$e$

$$
\alpha_{l t}=\sigma^{-i j\left(\alpha_{2}\right)^{-1}\left(\alpha_{l+1}\right)^{-1}\left(\alpha_{l+1}-e\right)} \alpha_{l+1}, 2 \leq l \leq d, l+1(\bmod d) .
$$

Então o grupo $\left\langle\sigma, \alpha_{1}, \alpha_{2}, \ldots, \alpha_{d}\right\rangle$ transitivo fechado por estado de grau $p^{2}$ é isomorfo a $G_{p, d}$.

\subsection{Representação fechada por estado de grau 4 de $G_{2,2}$}

Nesta seção, demonstramos que a representação de $G_{2,2}$ do Teorema 4.2.1 é finita por estado. Vamos supor, então, que $p=2, d=2$ e chamar $\alpha_{1}$ de $\alpha$ e $\alpha_{2}$ de $\beta$.

Corolário 4.3.1. Sejam

$$
\begin{gathered}
\sigma=(01)(23), \\
\alpha=\left(e, \sigma^{\beta^{-1}}, \beta, \sigma^{\beta^{-1}} \beta\right)(02)(13), \\
\beta=\left(\alpha, \alpha, \alpha, \sigma^{\beta^{-1}+\beta^{-1} \alpha^{-1}} \alpha\right) .
\end{gathered}
$$

Então $G_{2,2}$ é isomorfo ao grupo transitivo fechado por estado $\langle\sigma, \alpha, \beta\rangle$ de grau 4.

Demonstração. Basta aplicar o Teorema 4.2.1.

A demonstração de que $G_{2,2}$ é finito por estado segue provando que $\alpha$ tem 12 estados e esses estados geram $G_{2,2}$.

Teorema 4.3.2. Seja $\langle\sigma, \alpha, \beta\rangle$ a representação de $G_{2,2}$, dada no Corolário 4.3.1. Então o automorfismo $\alpha$ possui 12 estados e induz o autômato 


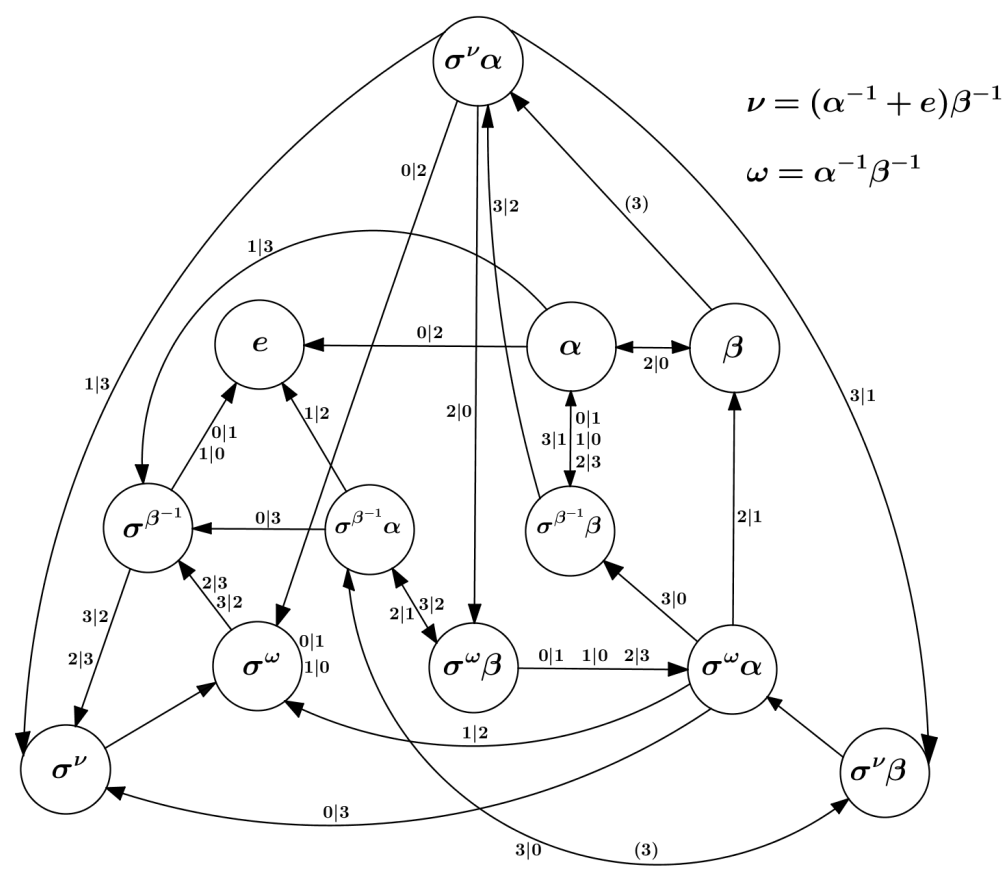

Figura 4.1: Autômato de $C_{2} \succ C^{2}$

que gera $G_{2,2}$.

Demonstração. Seja $n$ um número inteiro. Então

$$
\begin{gathered}
\alpha^{2 n}=\left(\beta^{n}, \beta^{n}, \beta^{n}, \sigma^{\beta^{-1}+\beta^{-(n+1)}} \beta^{n}\right), \\
\alpha^{2 n+1}=\left(\beta^{n}, \sigma^{\beta^{-(n+1)}} \beta^{n}, \beta^{n+1}, \sigma^{\beta^{-1}} \beta^{n+1}\right)(02)(13
\end{gathered}
$$

$\mathrm{e}$

$$
\beta^{n}=\left(\alpha^{n}, \alpha^{n}, \alpha^{n}, \sigma^{\beta^{-1}+\alpha^{-n} \beta^{-1}} \alpha^{n}\right) .
$$

Aplicando essas fórmulas nos conjugados de $\sigma$ obtemos

$$
\begin{gathered}
\sigma^{\alpha^{2 n} \beta^{m}}=\left(e, e, \sigma^{\alpha^{m} \beta^{n-1}+\beta^{-1}}, \sigma^{\alpha^{m} \beta^{n-1}+\beta^{-1}}\right)(01)(23), \\
\sigma^{\alpha^{2 n+1} \beta^{m}}=\left(\sigma^{\alpha^{m} \beta^{n}}, \sigma^{\alpha^{m} \beta^{n}}, \sigma^{\beta^{-1}}, \sigma^{\beta^{-1}}\right)(01)(23), \\
\sigma^{\alpha^{2 n} \beta^{m}} \alpha=\left(\sigma^{\beta^{-1}}, e, \sigma^{\alpha^{m} \beta^{n-1}} \beta, \sigma^{\alpha^{m} \beta^{n-1}+\beta^{-1}} \beta\right)(03)(12)
\end{gathered}
$$

e

$$
\sigma^{\alpha^{2 n+1} \beta^{m}} \alpha=\left(\sigma^{\alpha^{m} \beta^{n}+\beta^{-1}}, \sigma^{\alpha^{m} \beta^{n}}, \beta, \sigma^{\beta^{-1}} \beta\right)(03)(12)
$$


para todos números inteiros $m$ e $n$. Assim os estados de $\alpha$ são dados por

$$
\begin{aligned}
& \sigma^{\beta^{-1}}=\left(e, e, \sigma^{\alpha^{-1} \beta^{-1}+\beta^{-1}}, \sigma^{\alpha^{-1} \beta^{-1}+\beta^{-1}}\right)(01)(23), \\
& \sigma^{\beta^{-1}} \beta=\left(\alpha, \alpha, \alpha, \sigma^{\alpha^{-1} \beta^{-1}+\beta^{-1}} \alpha\right)(01)(23), \\
& \sigma^{\alpha^{-1} \beta^{-1}+\beta^{-1}}=\left(\sigma^{\alpha^{-1} \beta^{-1}}, \sigma^{\alpha^{-1} \beta^{-1}}, \sigma^{\alpha^{-1} \beta^{-1}}, \sigma^{\alpha^{-1} \beta^{-1}}\right) \\
& \sigma^{\alpha^{-1} \beta^{-1}+\beta^{-1}} \alpha=\left(\sigma^{\alpha^{-1} \beta^{-1}}, \sigma^{\alpha^{-1} \beta^{-1}+\beta^{-1}}, \sigma^{\alpha^{-1} \beta^{-1}} \beta, \sigma^{\alpha^{-1} \beta^{-1}+\beta^{-1}} \beta\right)(02)(13) \text {, } \\
& \sigma^{\alpha^{-1} \beta^{-1}}=\left(\sigma^{\alpha^{-1} \beta^{-1}}, \sigma^{\alpha^{-1} \beta^{-1}}, \sigma^{\beta^{-1}}, \sigma^{\beta^{-1}}\right)(01)(23), \\
& \sigma^{\alpha^{-1} \beta^{-1}} \beta=\left(\sigma^{\alpha^{-1} \beta^{-1}} \alpha, \sigma^{\alpha^{-1} \beta^{-1}} \alpha, \sigma^{\alpha^{-1} \beta^{-1}} \alpha, \sigma^{\beta^{-1}} \alpha\right)(01)(23) \text {, } \\
& \sigma^{\alpha^{-1} \beta^{-1}+\beta^{-1}} \beta=\left(\sigma^{\alpha^{-1} \beta^{-1}} \alpha, \sigma^{\alpha^{-1} \beta^{-1}} \alpha, \sigma^{\alpha^{-1} \beta^{-1}} \alpha, \sigma^{\beta^{-1}} \alpha\right) \text {, } \\
& \sigma^{\alpha^{-1} \beta^{-1}} \alpha=\left(\sigma^{\alpha^{-1} \beta^{-1}+\beta^{-1}}, \sigma^{\alpha^{-1} \beta^{-1}}, \beta, \sigma^{\beta^{-1}} \beta\right)(03)(12), \\
& \sigma^{\beta^{-1}} \alpha=\left(\sigma^{\beta^{-1}}, e, \sigma^{\beta^{-1}} \beta, \sigma^{\alpha^{-1} \beta^{-1}+\beta^{-1}} \beta\right)(03)(12) .
\end{aligned}
$$

Junto com $e, \alpha$ e $\beta$, esses são todos os estados de $\alpha$. Pondo $s_{0}=e, s_{1}=\alpha$, $s_{2}=\beta, s_{3}=\sigma^{\beta^{-1}}, s_{4}=\sigma^{\beta^{-1}} \alpha, s_{5}=\sigma^{\beta^{-1}} \beta, s_{6}=\sigma^{\alpha^{-1} \beta^{-1}}, s_{7}=\sigma^{\alpha^{-1} \beta^{-1}} \alpha$, $s_{8}=\sigma^{\alpha^{-1} \beta^{-1}} \beta, s_{9}=\sigma^{\alpha^{-1} \beta^{-1}+\beta^{-1}}, s_{10}=\sigma^{\alpha^{-1} \beta^{-1}+\beta^{-1}} \alpha$ e $s_{11}=\sigma^{\alpha^{-1} \beta^{-1}+\beta^{-1}} \beta, \mathrm{a}$ matriz de incidência do autômato $\alpha$ é

$$
\left(\begin{array}{ccccccccccccc} 
& e & s_{1} & s_{2} & s_{3} & s_{4} & s_{5} & s_{6} & s_{7} & s_{8} & s_{9} & s_{10} & s_{11} \\
e & 4 & & & & & & & & & & & \\
s_{1} & & 1 & 1 & 1 & & 1 & & & & & & \\
s_{2} & & 3 & & & & & & & & & 1 & \\
s_{3} & 2 & & & & & & & & & 2 & & \\
s_{4} & 1 & & & 1 & & & & & 1 & & & 1 \\
s_{5} & & 3 & & & & & & & & & 1 & \\
s_{6} & & & & 2 & & & 2 & & & & & \\
s_{7} & & & 1 & & & 1 & 1 & & & 1 & & \\
s_{8} & & & & & 1 & & & 3 & & & & \\
s_{9} & & & & & & & 4 & & & & & \\
s_{10} & & & & & & 1 & & 1 & 1 & & 1 \\
s_{11} & & & & & & & & & & & & \\
& & & & & & & & & & & &
\end{array}\right) .
$$

Aplique agora essa matriz e construa o autômato da Figura 4.1. 


\section{Referências}

[1] M. F. Atiyah, Elliptic operators, discrete groups and von Neumamm Algebras, Société Mathématic de France, (1976) 43 - 72.

[2] A. Berlatto and S. N. Sidki, Virtual endomorphisms of nilpotent groups, Groups, Geometry and Dynamics, vol.1, (2007), 21-46.

[3] A. M. Brunner and S. N. Sidki, Abelian state-closed subgroups of automorphisms of m-ary trees, Groups, Geometry, and Dynamics, 3 (2010) 455 $-472$.

[4] A. M. Brunner and S. N. Sidki, On the Automorphism group of the onerooted binary tree, Jornal of Algebra, 195 (1997) 455 - 486.

[5] L. Bartholdi and Z. Sunik, Some solvable automata groups, Contemp. Math. 394, (2006), 11 - 30.

[6] I. Bondarenko, D. D'Angeli and E. Rodaro, The Lamplighter group $Z_{3}$ 乙 $Z$ generated by a reversible automaton, ArXiv: 1502.06722.

[7] A. C. Dantas and S. N. Sidki, On state-closed representations of restricted wreath product of groups of type $G_{p, d}=C_{p} w r C^{d}$, ArXiv: 1505.05165.

[8] S. Eilenberg, Automata, Languages and Machines, Vol. A, Academia Prees, New York, (1974). 
[9] International GEOGEBRA Institute. GeoGebra. Software de Matemática 2016. Disponível em: http://www.geogebra.org/. Acesso em: 24 de janeiro de 2016.

[10] R. Grigorchuk, P-H Leemann and T. Nagnibeda, Lamplighter groups, de Bruijn graphs, spider-web graphs and their spectra, ArXiv:1502.06722.

[11] R. Grigorchuck, P. Linnel, Th. Schick and A. Zuk, On a conjecture of Atiyah, C.R. Acad. Sci. Paris, 331, Serie I, (2000) 663 - 668.

[12] R. Grigorchuck and A. Zuk, The Lamplighter group as a group generated by 2-state automaton and its spectrum, Geometriae Dedicata, 87, (2001) 209 244.

[13] W. Luck, $L^{2}$-invariantes: theory and applications to geometry and $K$ theory, Ergebnisse der Mathematik and ihrer Grenzgebiete, Springer-Verlag, Berlin, 2002.

[14] M. Kambides, P. Silva and B. Steinberg, The spectra of Lamplighter groups end Cayley machines, Geom. Dedicata, 120, (2006) 193 - 227.

[15] V. Kaimanovich and A. Vershik, Random walks on discrete groups: Boundary and Entropy, The Annals of Probability, 11, (1983) 457 - 490.

[16] M. Kapovich, Arithmetic aspects of self-similar groups, Groups Geometry and Dynamics 6 (2012), 737-754.

[17] Y. Muntyan and D. Savchuk, AutomGrp - GAP package for computations in self-similar groups and semigroups, Version 1.2.4 (2014).

[18] V. Nekrashevych, Self-similar groups, Math. Surveys and Monographs, 117, American Mathematical Society, Providence, RI, (2005).

[19] V. Nekrachevych and S. N. Sidki, Automorphisms of the binary tree: stateclosed subgroups and dynamics of 1/2 endomorphisms, In: Groups: London Mathematical Lecture Notes Series, Topological, Combinatorial and Arithmetic Aspects, Muller, T. W., (Ed.) 311, (2004) 375-404.

[20] L. Ribes and P. Zalesski, Profinite Groups, Springer-Verlag, Berlim, 1996.

[21] D. J. S. Robinson, A course in the theory of groups, Springer-Verlag, New York, (2000). 
[22] S. N. Sidki, Regular trees and their automorphisms, Monográfias de Matemática, vol 56, Instituto de Matemátuca Pura e Aplicada, 15, (1998).

[23] S. N. Sidki, Tree wreathing applied to the generation of groups by finite automata, International Journal of Algebra and Computation, 15. (2005) 18.

[24] P. Silva, B. Steinberg, On a class of automata groups generalizing Lamplighter groups, Int. J. Algebra Comut. 15, (2005) 1213-1234.

[25] A. Zuk, Automata Groups, Escola de Álgebra (Brazilian Algebra Meeting), Universidade Estadual de Maringá, (2014). 